\title{
2010
}

Lawrence Livermore National Laboratory

Annual IIIness and Injury Surveillance Report

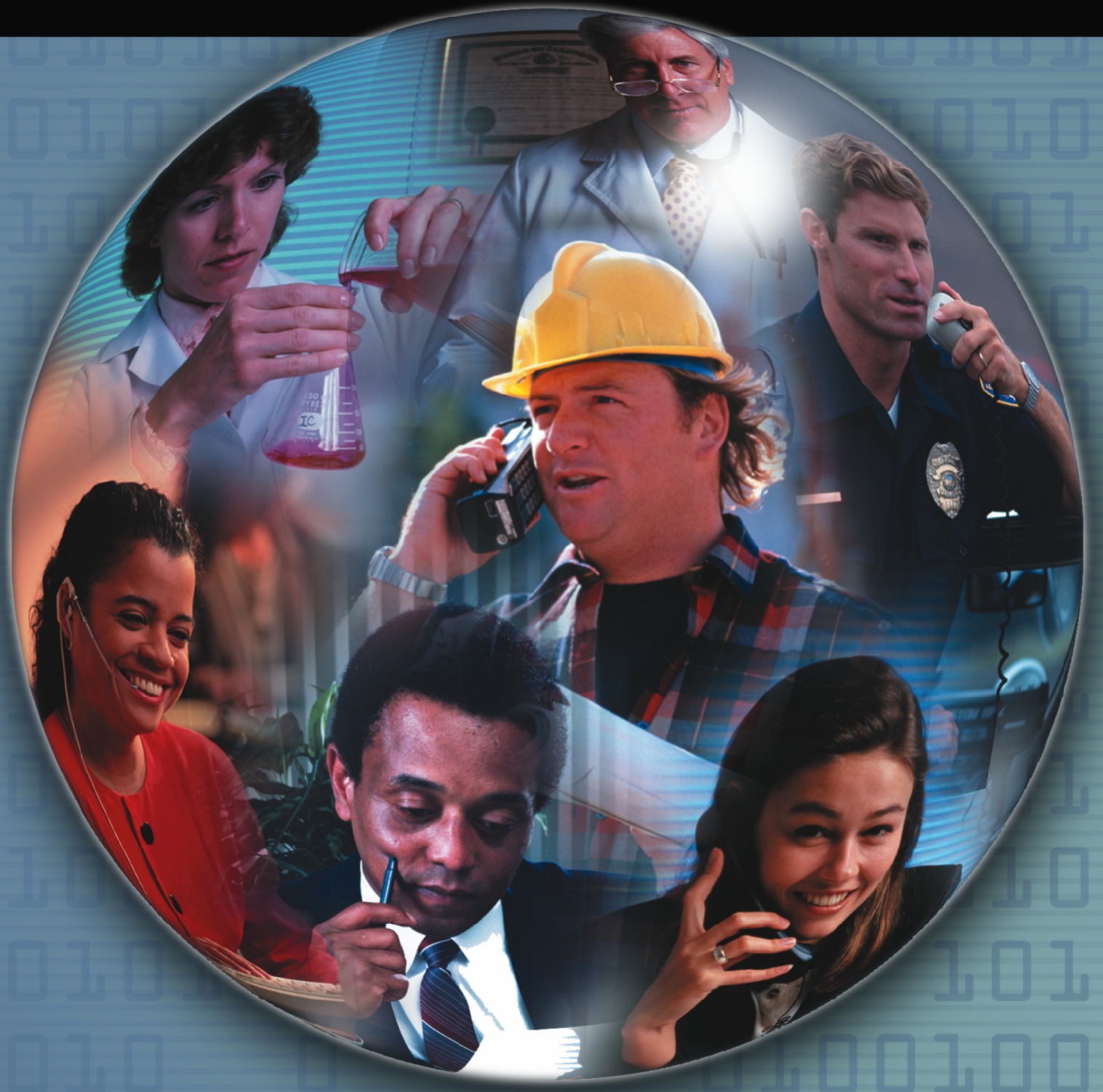




\section{Lawrence Livermore National Laboratory 2010 Illness and Injury Surveillance Report}

Questions or comments about this report or the Illness and Injury Surveillance Program (IISP) may be directed to:

E-mail:

Dr. Cliff Strader at cliff.strader@hq.doe.gov or Dr. Bonnie Richter at bonnie.richter@hq.doe.gov

or direct letters to:

Mail Stop HS-13 / GTN Building

U.S. Department of Energy

1000 Independence Avenue, S.W.

Washington, DC 20585-0270

Additional information about the Department of Energy's Office of Illness and Injury Prevention Programs, the IISP, and annual reports for DOE sites participating in this program can be found at:

http://www.hss.energy.gov/healthsafety/WSHP/epi/surv/

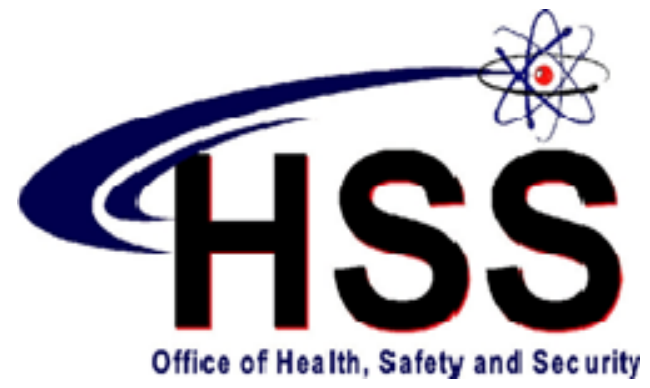

ACKNOWLEDGEMENT

LifeART images copyright 2000 Lippincott Williams \& Wilkins. All rights reserved.

This document was produced under contract number DE-AC05-06OR23100 between the U.S. Department of Energy and Oak Ridge Associated Universities. 


\title{
Lawrence Livermore National Laboratory 2010 Illness and Injury Surveillance Report
}

\author{
At A Glance
}

NOTE: In previous years, the number of days absent or lost and the age of the work force were reported as averages. Beginning in 2010, they are being reported as medians. A median is the value in a population that half the values exceed and half fall short of it. The median is less affected than the average when the distribution contains a few very large or very small values and provides a more representative summary measure.

A total of 6,168 LLNL employees were included in illness and injury surveillance in 2010; 1,865 (30 percent) women and 4,303 (70 percent) men. The work force has steadily decreased a total of 32 percent since 2006.

The 217 absences among 195 women resulted in an absence rate of 12 per 100 workers $(217 / 1,865)$. The 325 absences reported by 295 men resulted in an absence rate of 8 per 100 workers $(325 / 4,303)$. Absence rates have steadily increased since 2006 among women and men.

The median length of absences in 2010 was 12 days among both men and women, an increase of 1 day for men and a decrease of 1 day for women from 2006.

Disorders of the musculoskeletal (22 percent) and respiratory systems (19 percent) and unspecified symptoms (11 percent) accounted for 52 percent of all reported diagnoses among women. Musculoskeletal disorders (19 percent), respiratory conditions ( 18 percent), and injuries (16 percent) accounted for 53 percent of all reported diagnoses among men.

Respiratory diagnoses, musculoskeletal conditions, and injuries have typically been among the top 3 diagnostic categories reported by women and men at LLNL since 2006. This pattern is consistent with the overall observation of the top 3 diagnostic categories reported among sites participating in the Illness and Injury Surveillance Program (IISP) between 1999 and 2008. 
The highest absence rate among men was observed in Service workers, 12 absences per 100 workers. Among women, Security and Fire workers had the highest absence rate, 18 absences per 100 workers. Male Service workers also had the highest absence rates in 2007 and 2008; the last time female Security and Fire workers had the highest absence rate was in 2007.

Three definite and 7 possible sentinel health events for occupation (SHEO) diagnoses were reported in 2010; 5 diagnoses were carpal tunnel syndrome, all possible SHEOs. These diagnoses were reported by workers in the Professional, Administrative Support, and Technical Support occupational groups.

LLNL reported 31 OSHA-recordable events among women (2 events per 100 workers) and 44 OSHA-recordable events among men ( 1 event per 100 workers). Since 2006, the overall rates have held steady or declined, a pattern similar to what we have seen across the DOE Complex, where OSHA rates have been declining since 1999 .

The OSHA-recordable rate for women was highest among Professional workers; for men, the highest rate was in Technical Support workers. OSHA-recordable rates were highest among women aged 16 to 29 and men aged 50 and above, the exact opposite of what we saw in 2009.

Eighty-two percent of the OSHA health conditions involved musculoskeletal conditions or injuries, a pattern similar across all age groups.

All of the OSHA events reported by women were attributed to an accident, of which 17 were the result of repetitive trauma. Among men, 41 of the 44 OSHA events were attributed to an accident; 13 events were the result of overexertion and strenuous movement and 9 each were due to repetitive trauma and noise. 
The Lawrence Livermore National

Laboratory Work Force - 2010

The Work Force by Gender and Age . 1

The Work Force by Gender and Job

Category 1

\section{Number and Length of Absences}

Absence Rate by Gender and Age .. 2

Number of Days Absent by

Gender and Age .. 2

Absence Rate by Job Category

and Gender

Median Duration of Absence by

Job Category and Gender. .. 3

\section{Diagnostic Categories}

Number of Diagnoses and Lost Calendar Days by Diagnostic Category

(Categorized by ICD-9-CM) and Gender........ 4

Common Diagnoses Among Female

Workers in 2010 . 5

Common Diagnoses Among Male

Workers in 2010 .

Number of Most Frequently Reported

Diagnoses by Job Category and Gender 7

\section{Rates of Disease Occurrence}

Rates for All Illnesses and Injuries Combined by Job Category, Gender, and Age 8

Rates for Selected Diagnostic Categories by Job Category, Gender, and Age .8

\section{Time Trends}

Age-Adjusted Rates for All Diagnoses Combined Among Women and Men from 2006 to 2010
Age-Adjusted Rates for Selected Diagnostic Categories Among Women and Men from 2006 to 2010

Age-Adjusted Rates for All Diagnoses

Combined Among Women and Men by Job

Category from 2006 to $2010 \ldots \ldots \ldots \ldots \ldots \ldots \ldots \ldots . \ldots 12$

\section{Sentinel Health Events for Occupations (SHEOs)}

Characteristics of SHEOs by Gender 13

SHEO Diagnoses by Gender 13

Occupational Safety and Health Administration (OSHA)-Recordable Events

OSHA-Recordable Events by Gender and Age. 14

OSHA-Recordable Events by Job

Category and Gender 14

\section{Diagnostic and Accident Categories for OSHA-Recordable Events}

OSHA-Recordable Diagnoses by

Diagnostic Category and Gender

OSHA-Recordable Accidents by Type

and Gender.

\section{Rates of OSHA-Recordable Events}

OSHA-Recordable Rates by Age and Job

Categories Among Women, All Diagnoses

Combined

OSHA-Recordable Rates by Age and Job

Categories Among Men, All Diagnoses

Combined

Time Trends for OSHA-Recordable Events

Age-Adjusted Rates for All OSHA-Recordable Diagnoses Combined Among Women and Men by Job Category from 2006 to 2010 .

\section{Appendices}

Appendices A-W .20 
The Lawrence Livermore National Laboratory Work Force - 2010

Figure 1. The Work Force by Gender and Age

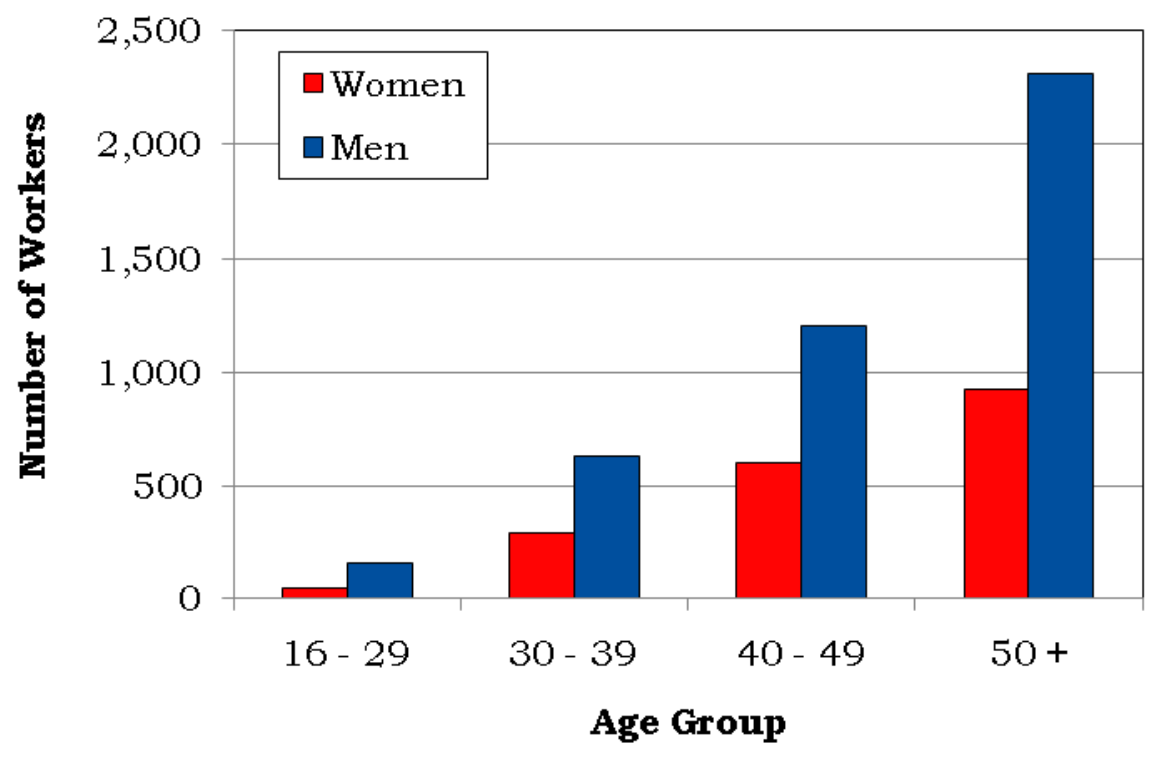

Figure 2. The Work Force by Gender and Job Category

\begin{tabular}{|c|c|c|}
\hline Job Category & Women & Men \\
\hline \multirow[t]{2}{*}{ Professional } & 537 & 2,605 \\
\hline & $29 \%$ & $61 \%$ \\
\hline \multirow{2}{*}{ Administrative Support } & 1,065 & 593 \\
\hline & $57 \%$ & $14 \%$ \\
\hline \multirow{2}{*}{ Technical Support } & 148 & 462 \\
\hline & $8 \%$ & $11 \%$ \\
\hline \multirow{2}{*}{ Service } & 92 & 194 \\
\hline & $5 \%$ & $4 \%$ \\
\hline \multirow{2}{*}{ Security and Fire } & 17 & 235 \\
\hline & $<1 \%$ & $5 \%$ \\
\hline \multirow{2}{*}{ Crafts } & 6 & 214 \\
\hline & $<1 \%$ & $5 \%$ \\
\hline \multirow{2}{*}{ Line Operators } & 0 & 0 \\
\hline & $0 \%$ & $0 \%$ \\
\hline Total & 1,865 & 4,303 \\
\hline
\end{tabular}




\section{Number and Length of Absences}

Figure 3. Absence Rate by Gender and Age

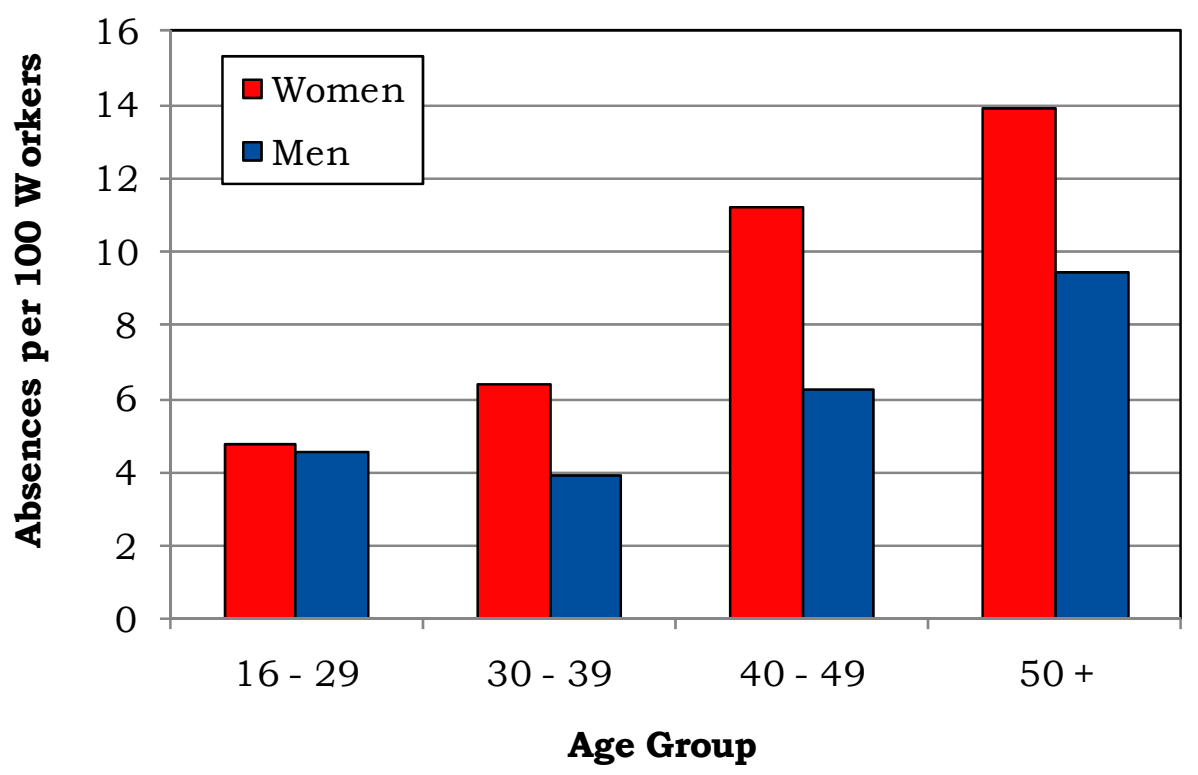

Figure 4. Number of Days Absent by Gender and Age

\begin{tabular}{|c|c|c|c|c|}
\hline \multirow{3}{*}{ Gender } & \multirow{2}{*}{ Age } & $\begin{array}{c}\text { Number of } \\
\text { Absences }\end{array}$ & \multicolumn{2}{|c|}{ Number of Days Absent } \\
\cline { 3 - 5 } & & 2 & Total & Median \\
\hline \multirow{4}{*}{ W omen* } & $16-29$ & 19 & 369 & 31 \\
\cline { 2 - 5 } & $30-39$ & 68 & 2,030 & 12 \\
\cline { 2 - 5 } & $40-49$ & 128 & 4,461 & 12 \\
\cline { 2 - 5 } & $50+$ & 217 & 6,921 & 12 \\
\cline { 2 - 5 } & Total & 7 & 165 & 11 \\
\hline \multirow{4}{*}{ Men } & $16-29$ & 25 & 431 & 10 \\
\cline { 2 - 5 } & $30-39$ & 75 & 1,858 & 12 \\
\cline { 2 - 5 } & $40-49$ & 218 & 7,387 & 13 \\
\cline { 2 - 5 } & $50+$ & 325 & 9,841 & 12 \\
\cline { 2 - 5 } & Total & & & \\
\hline
\end{tabular}

*Normal pregnancies were excluded from absences for women. 
Figure 5. Absence Rate by Job Category and Gender

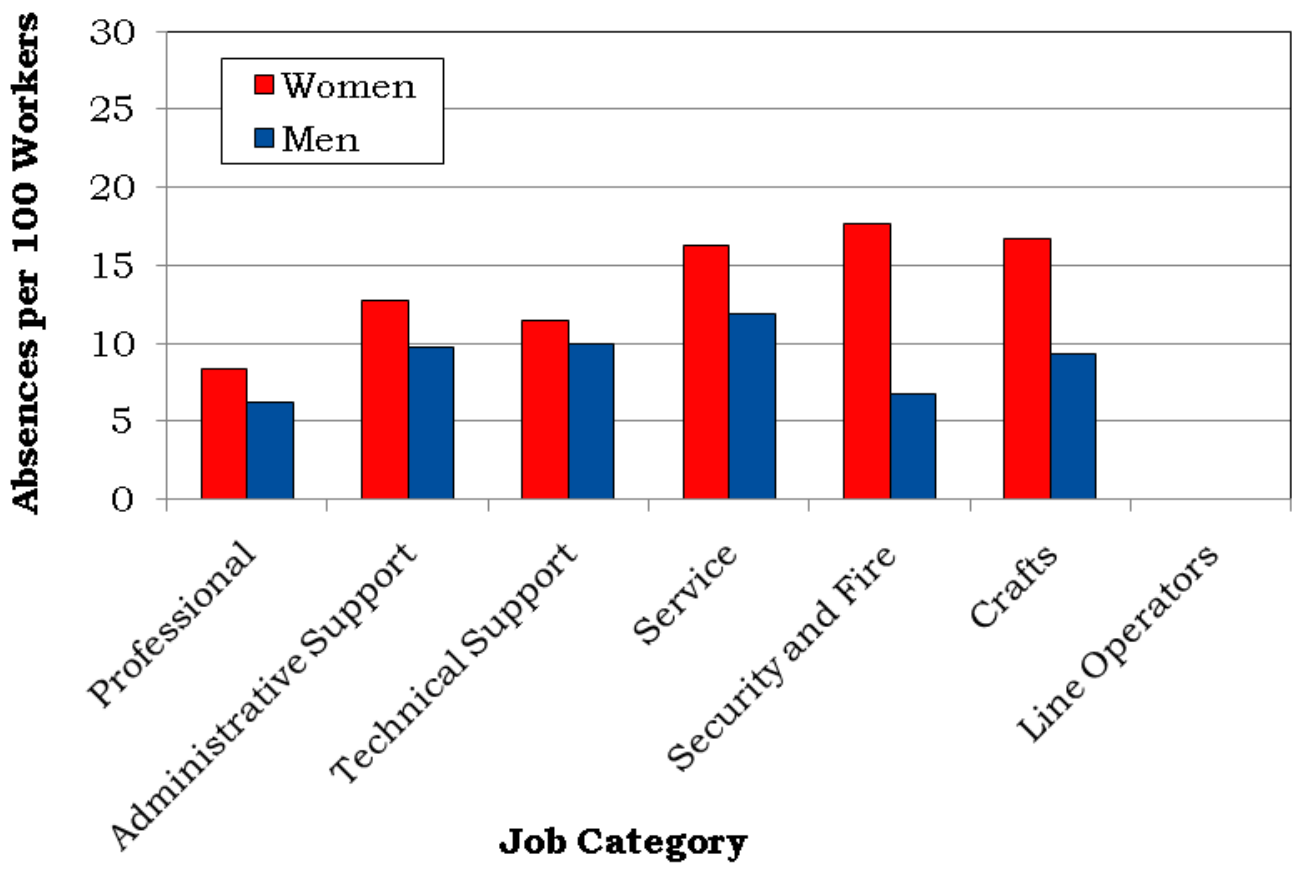

Figure 6. Median Duration of Absence by Job Category and Gender

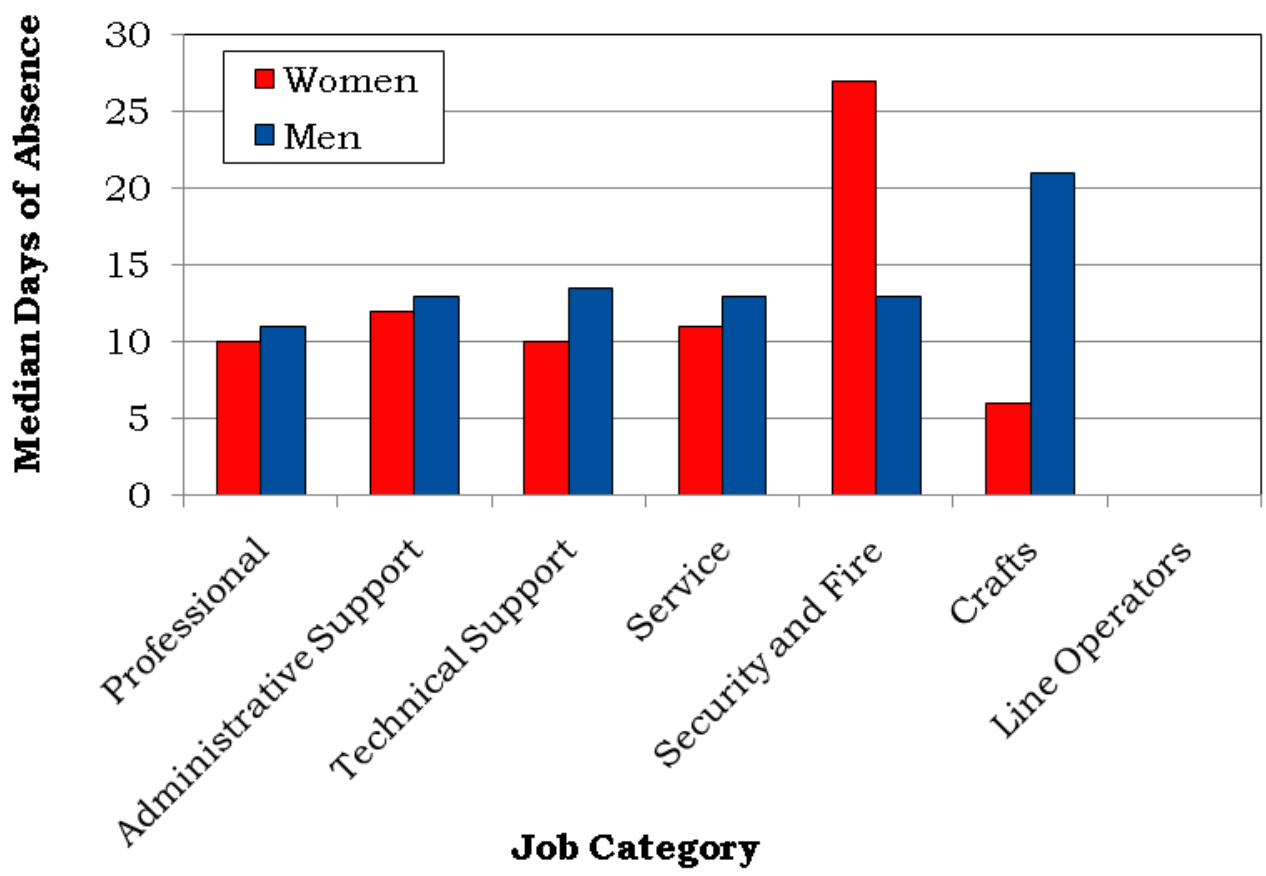




\section{Diagnostic Categories}

\section{Figure 7. Number of Diagnoses and Lost Calendar Days by Diagnostic Category (Categorized by ICD-9-CM) and Gender}

\begin{tabular}{|c|c|c|c|c|}
\hline \multirow[b]{2}{*}{ Diagnostic Category } & \multicolumn{2}{|c|}{ Women } & \multicolumn{2}{|c|}{ Men } \\
\hline & $\begin{array}{l}\text { Number of } \\
\text { Diagnoses }\end{array}$ & $\begin{array}{c}\text { Number } \\
\text { of Lost } \\
\text { Calendar } \\
\text { Days }\end{array}$ & $\begin{array}{l}\text { Number of } \\
\text { Diagnoses }\end{array}$ & $\begin{array}{c}\text { Number } \\
\text { of Lost } \\
\text { Calendar } \\
\text { Days }\end{array}$ \\
\hline Benign Growths & 5 & 179 & 5 & 103 \\
\hline Blood & 0 & 0 & 1 & 5 \\
\hline Cancer & 5 & 96 & 22 & 1,205 \\
\hline Digestive & 25 & 491 & 41 & 977 \\
\hline Endocrine / Me tabolic & 3 & 77 & 1 & 6 \\
\hline Existing Birth Condition & 0 & 0 & 0 & 0 \\
\hline Genitourinary & 20 & 719 & 10 & 95 \\
\hline Heart/Circulatory & 9 & 245 & 21 & 729 \\
\hline Infections/Parasites & 8 & 91 & 9 & 96 \\
\hline Injury & 25 & 769 & 59 & 1,824 \\
\hline Miscarriage & 1 & 12 & NA & NA \\
\hline Musculoskeletal & 52 & 2,528 & 69 & 3,366 \\
\hline Nervous System & 10 & 291 & 21 & 364 \\
\hline Psychological & 1 & 24 & 5 & 315 \\
\hline Respiratory & 45 & 942 & 64 & 860 \\
\hline Skin & 2 & 10 & 3 & 42 \\
\hline Unspecified Symptoms & 28 & 1,086 & 27 & 423 \\
\hline
\end{tabular}

Note: Lost calendar days for each absence are counted more than once when multiple diagnoses occur in different diagnostic categories for the same absence. 


\section{Figure 8. Common Diagnoses Among Female Workers in 2010}

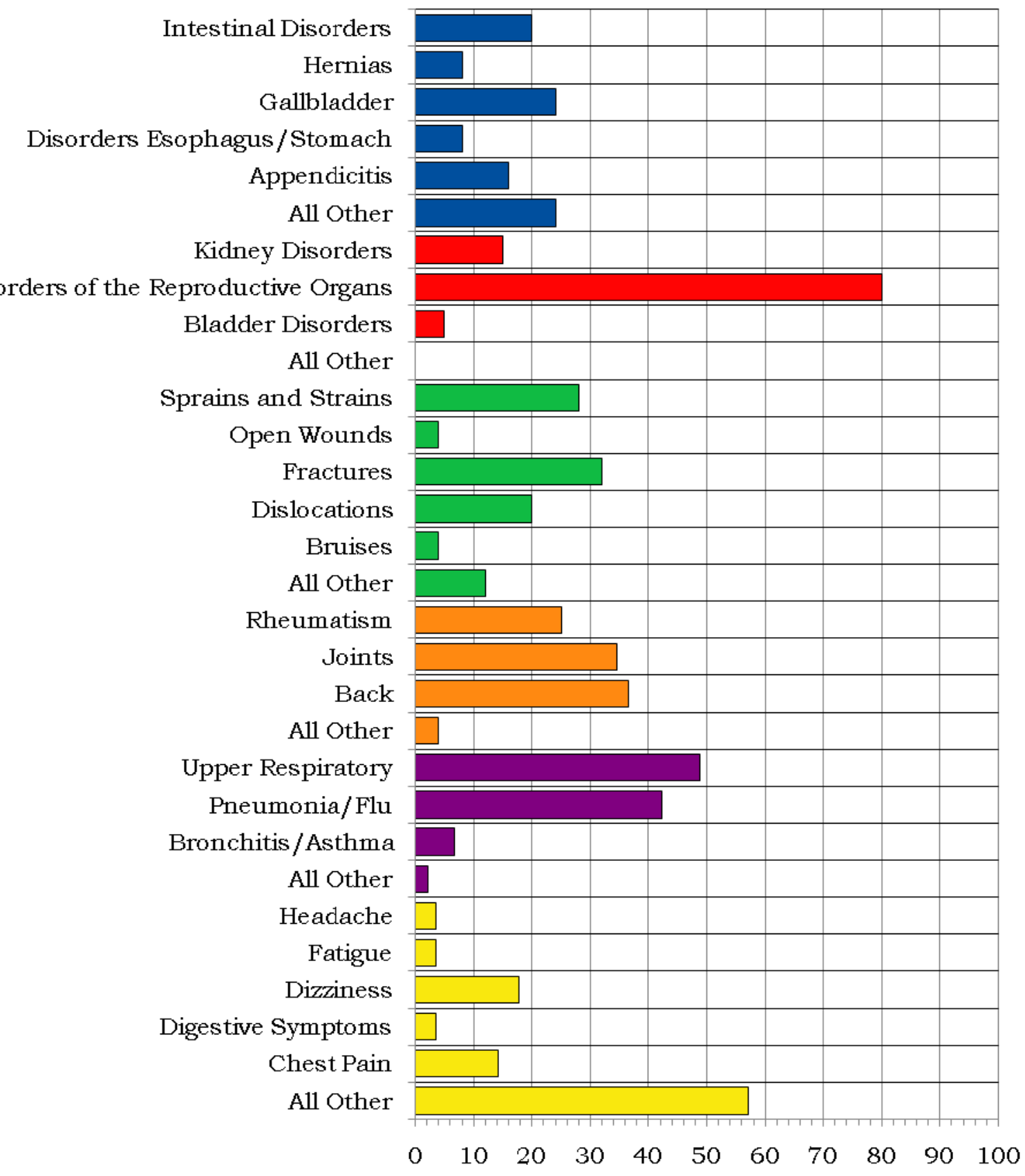

Percent Distribution of Diagnoses Within Diagnostic Category

Dige stive, 25 Diagnoses

Genitourinary, 20 Diagnoses

Injury, 25 Diagnoses
Musculoskeletal, 52 Diagnoses

Respiratory, 45 Diagnoses

Unspecified Symptoms, 28 Diagnoses 


\section{Figure 9. Common Diagnoses Among Male Workers in 2010}

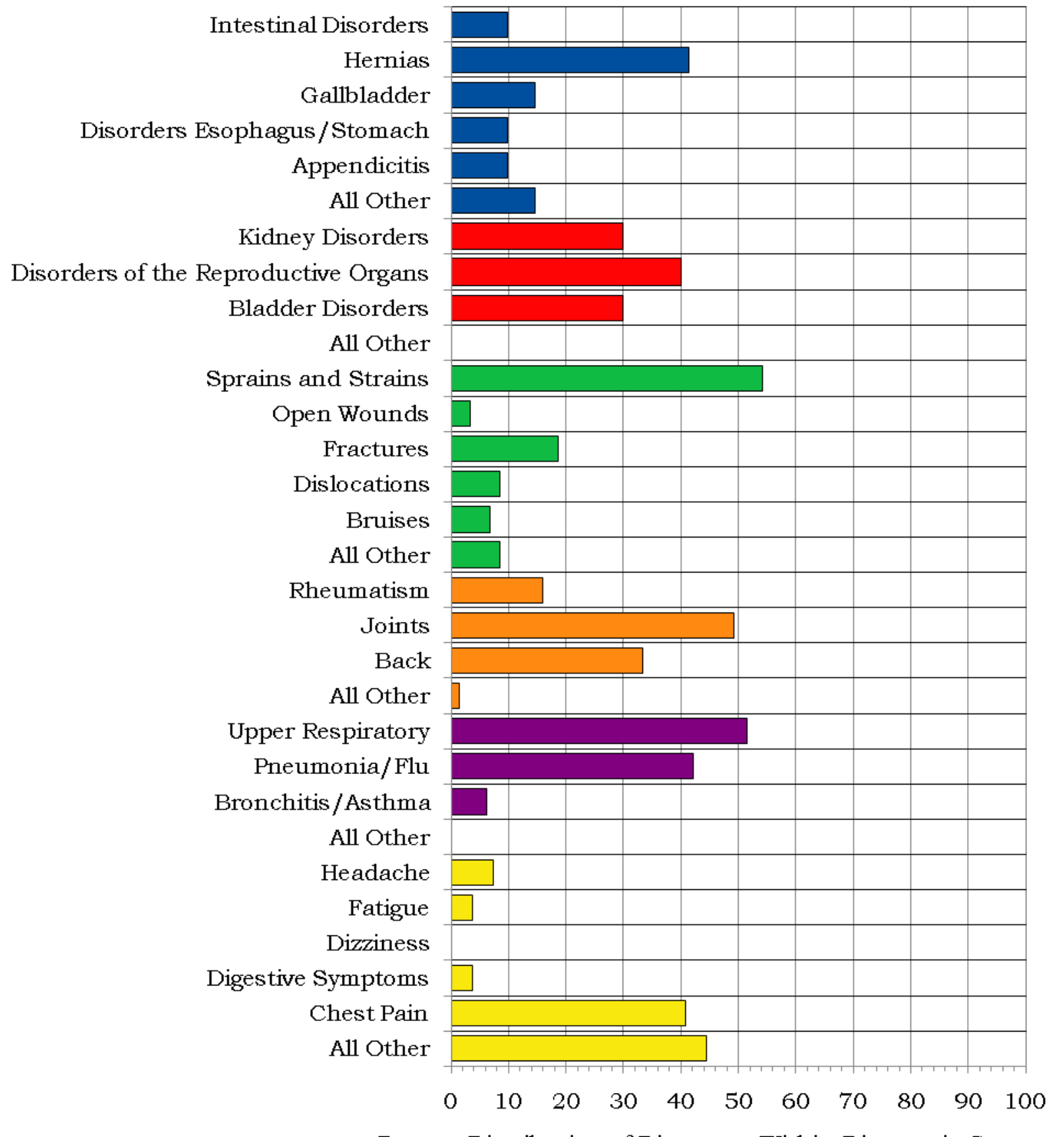

Percent Distribution of Diagnoses Within Diagnostic Category

Dige stive, 41 Diagnoses

Genitourinary, 10 Diagnoses

Injury, 59 Diagnoses
Musculoskeletal, 69 Diagnoses

Respiratory, 64 Diagnoses

Unspecified Symptoms, 27 Diagnoses 


\section{Figure 10. Number of Most Frequently Reported Diagnoses by Job Category and Gender}

\begin{tabular}{|c|c|c|c|c|}
\hline Job Category & Men & & Women & \\
\hline \multirow{5}{*}{ Professional } & Respiratory & 34 & M usculoskeletal & 11 \\
\hline & M usculoskeletal & 31 & Respiratory & 9 \\
\hline & Injury & 24 & Digestive & 5 \\
\hline & & & Injury & 5 \\
\hline & & & Unspecified Symptoms & 5 \\
\hline \multirow{4}{*}{ Administrative Support } & M usculoskeletal & 16 & M usculoskeletal & 30 \\
\hline & Respiratory & 11 & Respiratory & 28 \\
\hline & Injury & 7 & Unspecified Symptoms & 19 \\
\hline & Digestive & 7 & & \\
\hline \multirow{5}{*}{ Technical Support } & Injury & 14 & Respiratory & 5 \\
\hline & Respiratory & 8 & Unspecified Symptoms & 3 \\
\hline & M usculoskeletal & 7 & M usculoskeletal & 2 \\
\hline & Unspecified Symptoms & 7 & Injury & 2 \\
\hline & & & Genitourinary & 2 \\
\hline \multirow{4}{*}{ Service } & Injury & 5 & M usculoskeletal & 4 \\
\hline & Digestive & 5 & Respiratory & 3 \\
\hline & Respiratory & 4 & Injury & 2 \\
\hline & Musculoskeletal & 4 & & \\
\hline \multirow{3}{*}{ Security and Fire } & Respiratory & 4 & M usculoskeletal & 3 \\
\hline & M usculoskeletal & 4 & & \\
\hline & Injury & 4 & & \\
\hline \multirow{3}{*}{ Crafts } & M usculoskeletal & 7 & M usculoskeletal & 2 \\
\hline & Injury & 5 & & \\
\hline & Respiratory & 3 & & \\
\hline Line Operators & & $\mathbf{0}$ & & 0 \\
\hline
\end{tabular}




\section{Rates of Disease Occurrence}

Figure 11. Rates for All Illnesses and Injuries Combined by Job Category, Gender, and Age

\begin{tabular}{|c|c|c|c|c|}
\hline \multirow{2}{*}{$\begin{array}{c}\text { All Illnesses \& } \\
\text { Injuries Combined }\end{array}$} & \multicolumn{4}{|c|}{ Rate per 1,000} \\
\hline & Job Category & Age & Men & Women \\
\hline & \multirow{2}{*}{ Professional } & $<50$ & 44 & 86 \\
\hline & & $50+$ & 84 & 81 \\
\hline & \multirow{2}{*}{ Administrative Support } & $<50$ & 72 & 97 \\
\hline & & $50+$ & 137 & 192 \\
\hline & \multirow{2}{*}{ Technical Support } & $<50$ & 96 & 118 \\
\hline & & $50+$ & 141 & 127 \\
\hline & \multirow{2}{*}{ Service } & $<50$ & 85 & 125 \\
\hline & & $50+$ & 170 & 183 \\
\hline & \multirow{2}{*}{ Security and Fire } & $<50$ & 61 & 286 \\
\hline & & $50+$ & 107 & 100 \\
\hline & \multirow{2}{*}{ Crafts } & $<50$ & 75 & 667 \\
\hline & & $50+$ & 124 & 0 \\
\hline & \multirow{2}{*}{ Line Operators } & $<50$ & 0 & 0 \\
\hline & & $50+$ & 0 & 0 \\
\hline
\end{tabular}

Figure 12. Rates for Selected Diagnostic Categories by Job Category, Gender, and Age

\begin{tabular}{|c|c|c|c|c|}
\hline \multirow{2}{*}{ Cancer } & \multicolumn{4}{|c|}{ Rate per 1,000} \\
\hline & Job Category & Age & Men & Women \\
\hline & Profeccional & $<50$ & 0 & 0 \\
\hline & FIOICSSIOHAl & $50+$ & 10 & 4 \\
\hline & Adminictrotive Sunnort & $<50$ & 0 & 4 \\
\hline & 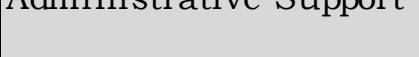 & $50+$ & 9 & 2 \\
\hline & Technical Sunnort & $<50$ & 0 & 0 \\
\hline & I Ecrimical support & $50+$ & 13 & 0 \\
\hline & Serrise & $<50$ & 0 & 0 \\
\hline & 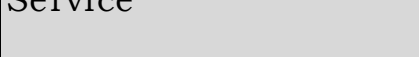 & $50+$ & 9 & 17 \\
\hline & Security and Fire & $<50$ & 0 & 0 \\
\hline & Security and Fire & $50+$ & 0 & $\mathbf{0}$ \\
\hline & Crafts & $<50$ & 0 & 0 \\
\hline & & $50+$ & 8 & 0 \\
\hline & Line Operators & $<50$ & 0 & 0 \\
\hline & & $50+$ & 0 & 0 \\
\hline
\end{tabular}


Figure 12. Rates for Selected Diagnostic Categories by Job Category, Gender, and Age (Continued)

\begin{tabular}{|l|l|c|c|c|}
\hline \multirow{2}{*}{ Heart/Circulatory } & \multicolumn{4}{|c|}{ Rate per 1,000 } \\
\cline { 2 - 5 } & \multicolumn{1}{|c|}{ Job Category } & Age & M en & W omen \\
\cline { 2 - 5 } & Profe ssional & $<50$ & $\mathbf{1}$ & $\mathbf{0}$ \\
\cline { 2 - 5 } & $50+$ & $\mathbf{6}$ & $\mathbf{9}$ \\
\cline { 2 - 5 } & Administrative Support & $<50$ & $\mathbf{0}$ & $\mathbf{2}$ \\
\cline { 2 - 5 } & $50+$ & $\mathbf{9}$ & $\mathbf{9}$ \\
\cline { 2 - 5 } & \multirow{2}{*}{ Technical Support } & $<50$ & $\mathbf{4}$ & $\mathbf{0}$ \\
\cline { 2 - 5 } & $50+$ & $\mathbf{2 1}$ & $\mathbf{0}$ \\
\cline { 2 - 5 } & \multirow{2}{*}{ Service } & $<50$ & $\mathbf{0}$ & $\mathbf{0}$ \\
\cline { 2 - 5 } & $50+$ & $\mathbf{9}$ & $\mathbf{1 7}$ \\
\cline { 2 - 5 } & \multirow{2}{*}{ Security and Fire } & $<50$ & $\mathbf{0}$ & $\mathbf{0}$ \\
\cline { 2 - 5 } & $50+$ & $\mathbf{0}$ & $\mathbf{0}$ \\
\cline { 2 - 5 } & \multirow{2}{*}{ Crafts } & $<50$ & $\mathbf{1 1}$ & $\mathbf{0}$ \\
\cline { 2 - 5 } & $50+$ & $\mathbf{0}$ & $\mathbf{0}$ \\
\cline { 2 - 5 } & \multirow{2}{*}{ Line Operators } & $<50$ & $\mathbf{0}$ & $\mathbf{0}$ \\
\cline { 2 - 5 } & $50+$ & $\mathbf{0}$ & $\mathbf{0}$ \\
\hline
\end{tabular}

\begin{tabular}{|c|c|c|c|c|}
\hline \multirow{2}{*}{ Respiratory } & \multicolumn{4}{|c|}{ Rate per 1,000} \\
\hline & Job Category & Age & Men & Women \\
\hline & \multirow{2}{*}{ Professional } & $<50$ & 10 & 13 \\
\hline & & $50+$ & 15 & 21 \\
\hline & \multirow{2}{*}{ Administrative Support } & $<50$ & 20 & 23 \\
\hline & & $50+$ & 18 & 29 \\
\hline & \multirow{2}{*}{ Technical Support } & $<50$ & 22 & 35 \\
\hline & & $50+$ & 13 & 32 \\
\hline & \multirow{2}{*}{ Service } & $<50$ & 24 & 31 \\
\hline & & $50+$ & 18 & 33 \\
\hline & \multirow{2}{*}{ Security and Fire } & $<50$ & 11 & 0 \\
\hline & & $50+$ & 36 & 0 \\
\hline & \multirow{2}{*}{ Crafts } & $<50$ & 22 & 0 \\
\hline & & $50+$ & 8 & 0 \\
\hline & \multirow{2}{*}{ Line Operators } & $<50$ & 0 & 0 \\
\hline & & $50+$ & 0 & 0 \\
\hline
\end{tabular}


Figure 12. Rates for Selected Diagnostic Categories by Job Category, Gender, and Age (Continued)

\begin{tabular}{|c|c|c|c|c|}
\hline \multirow{2}{*}{ Injury } & \multicolumn{4}{|c|}{ Rate per 1,000} \\
\hline & Job Category & Age & Men & Women \\
\hline & \multirow{2}{*}{ Professional } & $<50$ & 6 & 10 \\
\hline & & $50+$ & 12 & 9 \\
\hline & \multirow{2}{*}{ Administrative Support } & $<50$ & 20 & 10 \\
\hline & & $50+$ & 6 & 20 \\
\hline & \multirow{2}{*}{ Technical Support } & $<50$ & 31 & 12 \\
\hline & & $50+$ & 30 & 16 \\
\hline & \multirow{2}{*}{ Service } & $<50$ & 49 & 31 \\
\hline & & $50+$ & 9 & 17 \\
\hline & \multirow{2}{*}{ Security and Fire } & $<50$ & 17 & 0 \\
\hline & & $50+$ & 18 & 0 \\
\hline & \multirow{2}{*}{ Crafts } & $<50$ & 11 & 0 \\
\hline & & $50+$ & 33 & 0 \\
\hline & \multirow{2}{*}{ Line Operators } & $<50$ & 0 & 0 \\
\hline & & $50+$ & 0 & 0 \\
\hline
\end{tabular}

\section{Time Trends}

Figure 13. Age-Adjusted Rates for All Diagnoses Combined Among Women and Men from 2006 to 2010*

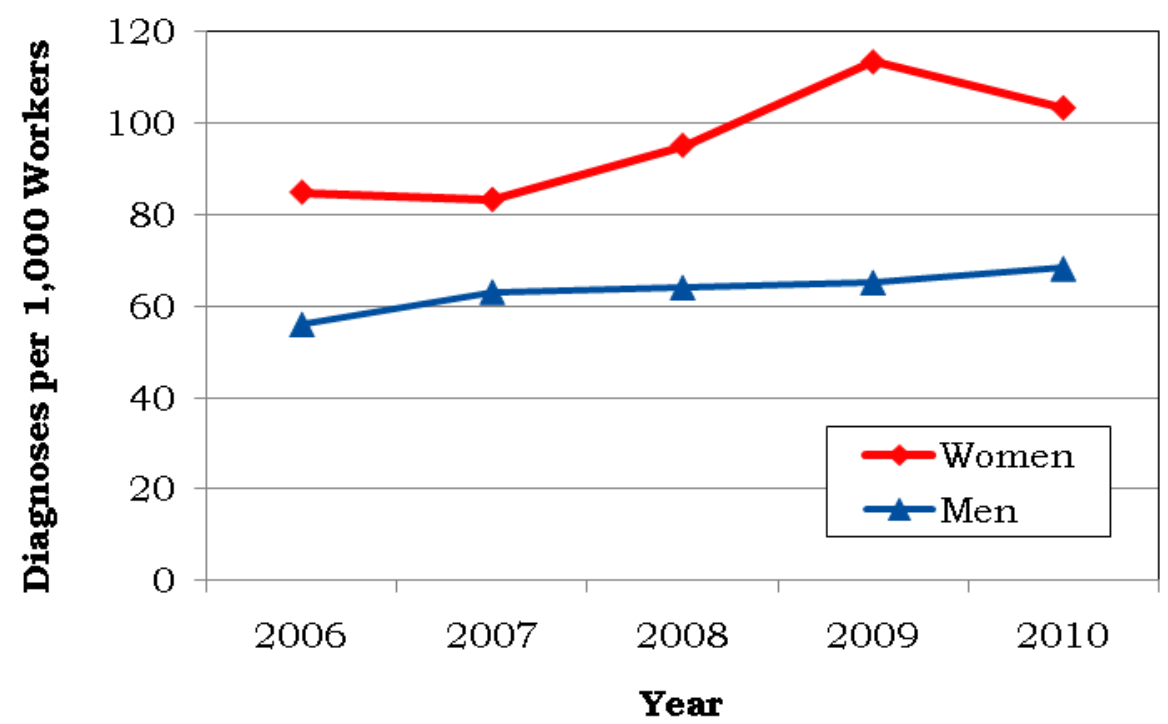

*Standardized to age distribution of 2000 U.S. population. 
Figure 14. Age-Adjusted Rates for Selected Diagnostic Categories Among Women and Men from 2006 to 2010*

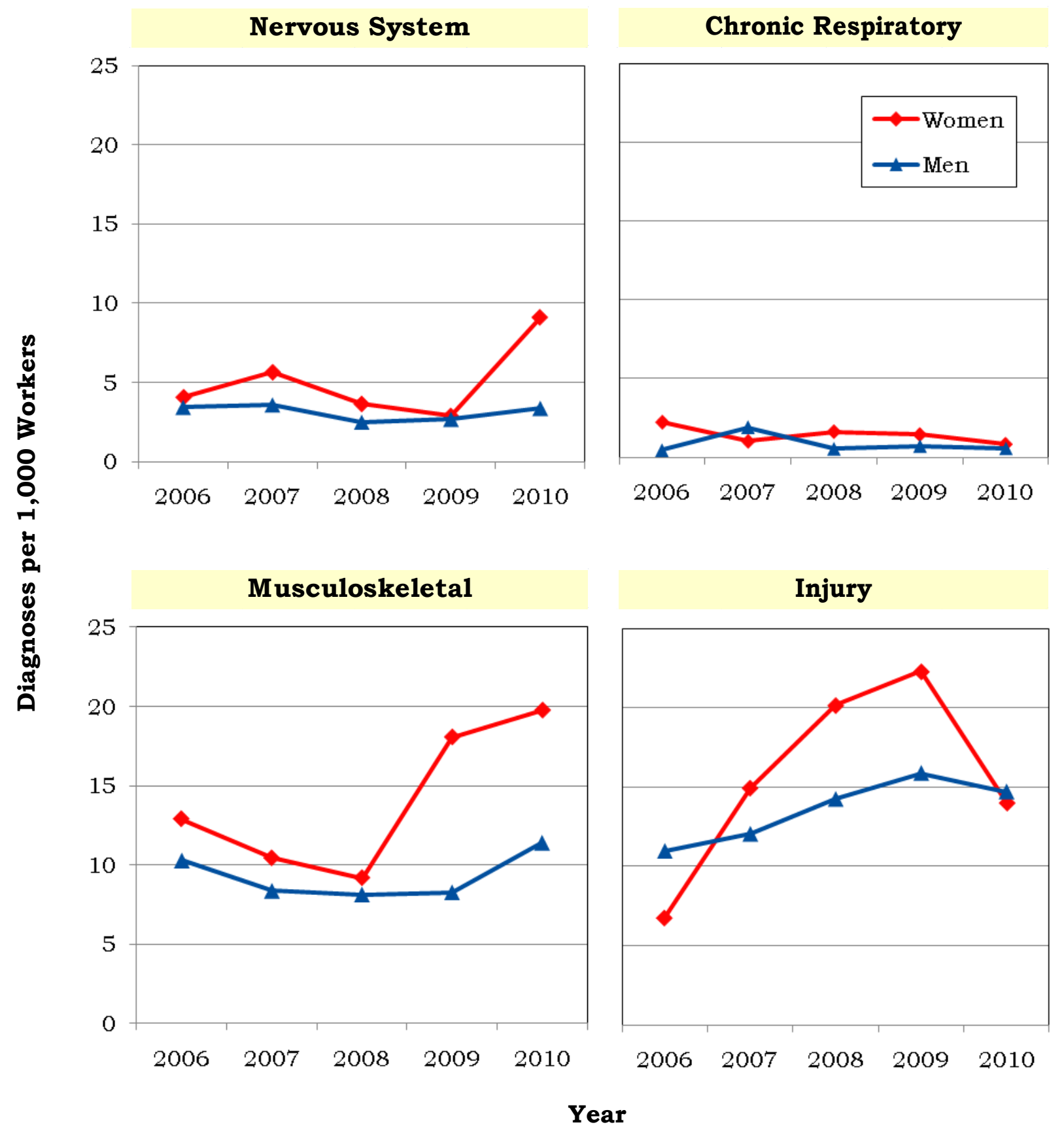

*Standardized to age distribution of 2000 U.S. population. 
Figure 15. Age-Adjusted Rates for All Diagnoses Combined Among Women and Men by Job Category from 2006 to 2010*

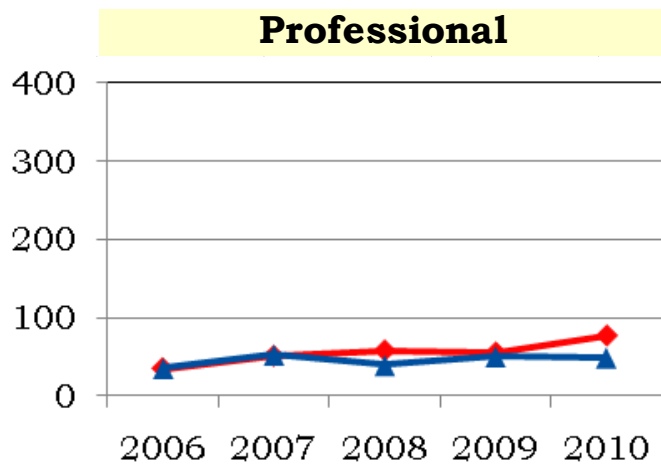

$\sim$ Women

$\mp$ Men

Administrative Support

مै

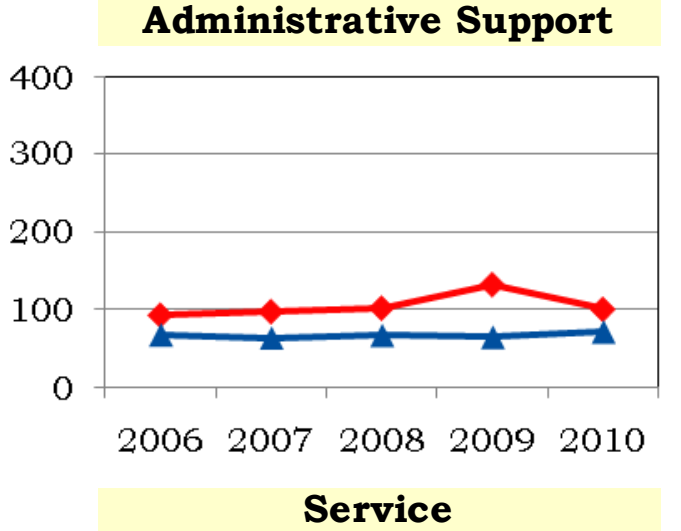

Technical Support

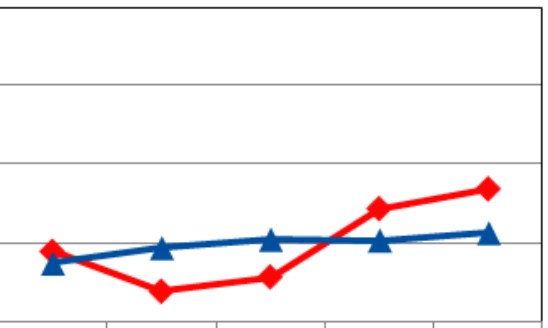

20062007200820092010

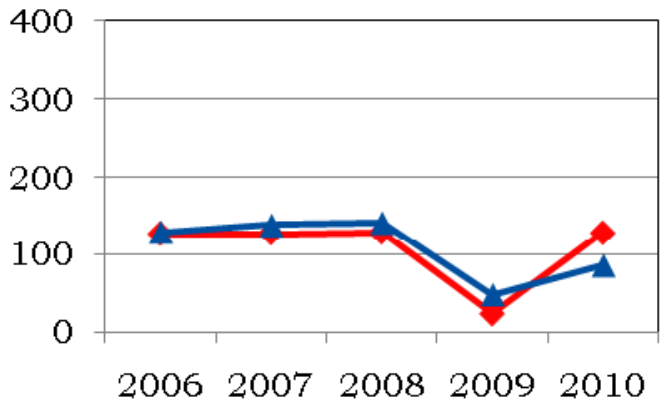

Security and Fire

Crafts
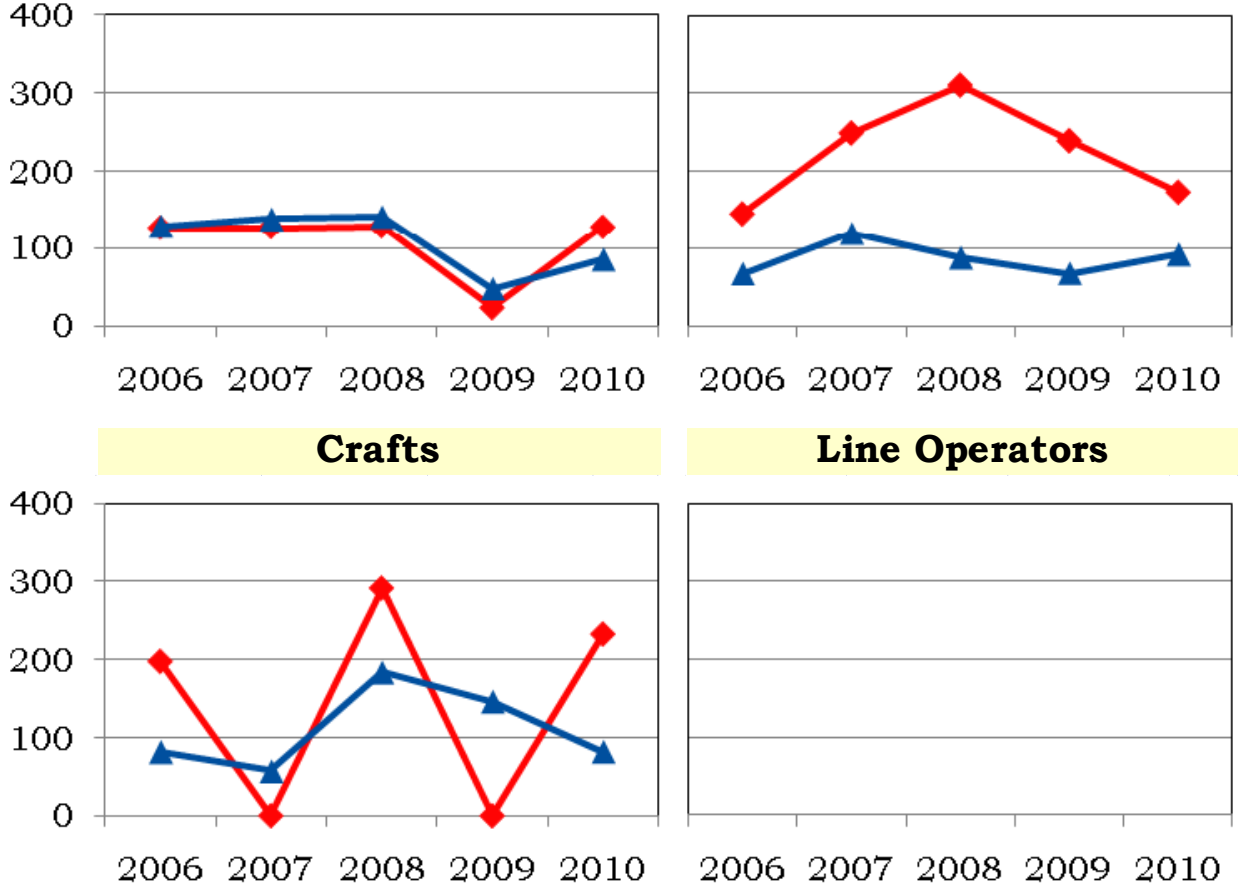

20062007200820092010

Line Operators

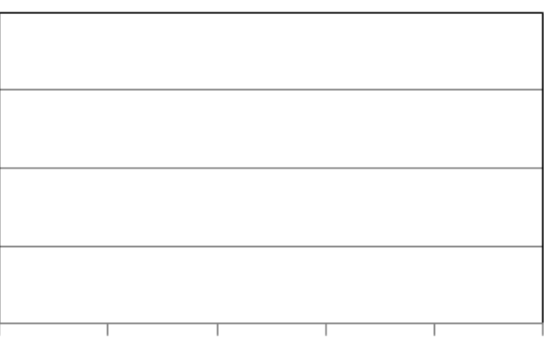

20062007200820092010

\section{Year}

*Standardized to age distribution of 2000 U.S. population. 


\section{Sentinel Health Events for Occupations (SHEOs)}

An occupational sentinel health event (SHEO) is a disease, disability, or death that is likely to be occupationally related. Although sentinel health events may indicate an occupational exposure, many may result from nonoccupational exposures. Sentinel health events are therefore assessed in two categories:

Definite Sentinel Health Events: Diseases that are unlikely to occur in the absence of an occupational exposure (e.g., asbestosis).

Possible Sentinel Health Events: Diseases that may be occupational but can also occur in the absence of an occupational exposure (e.g., lung cancer or carpal tunnel syndrome).

Figure 16. Characteristics of SHEOs by Gender

\begin{tabular}{|l|c|c|c|c|}
\hline \multirow{2}{*}{} & \multicolumn{2}{|c|}{$\begin{array}{c}\text { Total Number of } \\
\text { SHEO Diagnoses }\end{array}$} & \multicolumn{2}{c|}{$\begin{array}{c}\text { Total Number of } \\
\text { Days Absent }\end{array}$} \\
\cline { 2 - 5 } & M en & Women & M en & W omen \\
\hline Definite & 3 & 0 & 54 & 0 \\
\hline Possible & 4 & 3 & 320 & 161 \\
\hline Total & 7 & 3 & 374 & 161 \\
\hline
\end{tabular}

Figure 17. SHEO Diagnoses by Gender

\begin{tabular}{|l|c|c|}
\hline \multirow{2}{*}{ Diagnoses } & \multicolumn{2}{c|}{ Gender } \\
\cline { 2 - 3 } & Women & M en \\
\hline Carpal Tunnel Syndrome & 3 & 2 \\
\hline Other Conditions & 0 & 5 \\
\hline
\end{tabular}


Occupational Safety and Health Administration (OSHA)-Recordable Events

Figure 18. OSHA-Recordable Events by Gender and Age

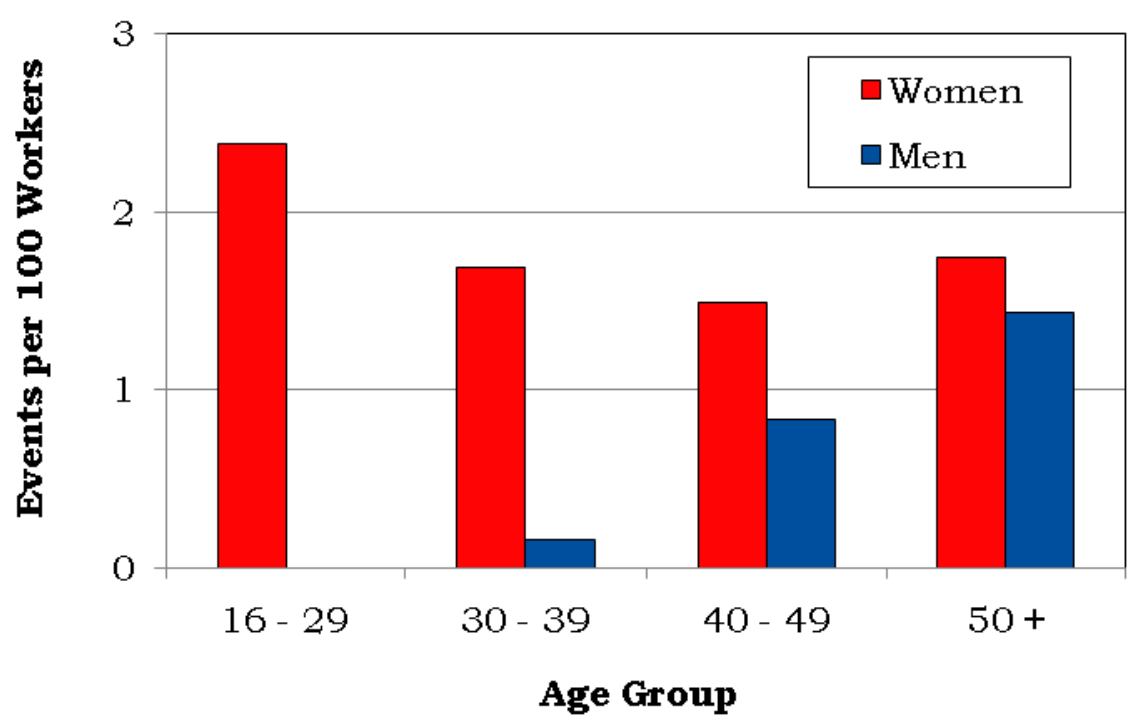

Figure 19. OSHA-Recordable Events by Job Category and Gender

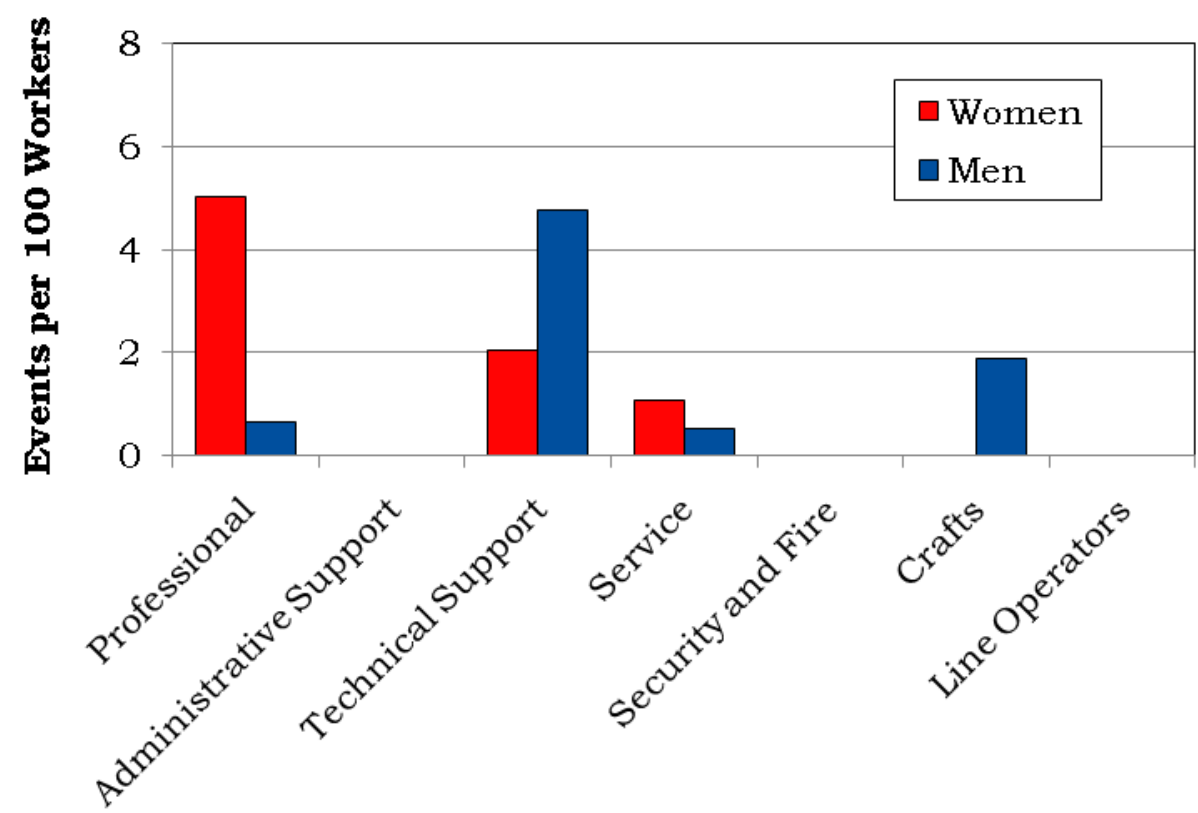

Job Category 


\section{Diagnostic and Accident Categories for OSHA-Recordable Events}

Figure 20. OSHA-Recordable Diagnoses by Diagnostic Category and Gender

\begin{tabular}{|l|c|c|}
\hline \multirow{2}{*}{ Diagnostic Category } & \multicolumn{2}{c|}{ Gender } \\
\cline { 2 - 3 } & Women & M en \\
\hline Musculoskeletal & $\mathbf{2 5}$ & $\mathbf{2 7}$ \\
\hline Nervous System & $\mathbf{3}$ & $\mathbf{1 1}$ \\
\hline Skin & $\mathbf{0}$ & $\mathbf{3}$ \\
\hline Unspecified Symptoms & $\mathbf{6}$ & $\mathbf{2}$ \\
\hline Injury & $\mathbf{3 1}$ & $\mathbf{3 0}$ \\
\hline Fractures - Lower Limb & $\mathbf{2}$ & $\mathbf{1}$ \\
\hline Back Sprains \& Strains & $\mathbf{1}$ & $\mathbf{5}$ \\
\hline Other Sprains \& Strains & $\mathbf{4}$ & $\mathbf{7}$ \\
\hline Open Wounds - Upper Limb & $\mathbf{0}$ & $\mathbf{4}$ \\
\hline Open Wounds - Lower Limb & $\mathbf{1}$ & $\mathbf{0}$ \\
\hline Superficial Injuries & $\mathbf{1}$ & $\mathbf{0}$ \\
\hline Bruises & $\mathbf{3}$ & $\mathbf{1}$ \\
\hline Burns & $\mathbf{1}$ & $\mathbf{0}$ \\
\hline Unspecified Injuries & $\mathbf{1 7}$ & $\mathbf{1 0}$ \\
\hline Poisonings by Drugs & $\mathbf{0}$ & $\mathbf{1}$ \\
\hline Adverse Reactions to Non-Medical Substances & $\mathbf{1}$ & $\mathbf{1}$ \\
\hline
\end{tabular}


Figure 21. OSHA-Recordable Accidents by Type and Gender

\begin{tabular}{|l|c|c|}
\hline \multirow{2}{*}{ Accident Category } & \multicolumn{2}{c|}{ Gender } \\
\cline { 2 - 3 } & Women & M en \\
\cline { 2 - 3 } & $\begin{array}{c}\text { Number of } \\
\text { Accidents }\end{array}$ & $\begin{array}{c}\text { Number of } \\
\text { Accidents }\end{array}$ \\
\hline Non Motor Vehicle & $\mathbf{1}$ & $\mathbf{1}$ \\
\hline Overdose / Wrong Drug & $\mathbf{0}$ & $\mathbf{1}$ \\
\hline Poisoning - Non-Medicinal & $\mathbf{1}$ & $\mathbf{0}$ \\
\hline Falls & $\mathbf{6}$ & $\mathbf{3}$ \\
\hline Natural/Environmental Factors & $\mathbf{0}$ & $\mathbf{1}$ \\
\hline Other Accidents & $\mathbf{2 3}$ & $\mathbf{3 5}$ \\
\hline Struck by an Object & $\mathbf{2}$ & $\mathbf{0}$ \\
\hline Caught Between Objects & $\mathbf{0}$ & $\mathbf{1}$ \\
\hline Machinery & $\mathbf{0}$ & $\mathbf{1}$ \\
\hline Cutting/Piercing Instrument/Object & $\mathbf{0}$ & $\mathbf{2}$ \\
\hline Hot, Corrosive, or Caustic Material/Steam & $\mathbf{1}$ & $\mathbf{0}$ \\
\hline Overexertion/Strenuous Movements & $\mathbf{3}$ & $\mathbf{1 3}$ \\
\hline Repetitive Trauma & $\mathbf{1 7}$ & $\mathbf{9}$ \\
\hline Noise & $\mathbf{0}$ & $\mathbf{9}$ \\
\hline Total & $\mathbf{3 1}$ & $\mathbf{4 1}$ \\
\hline
\end{tabular}




\section{Rates of OSHA-Recordable Events}

Figure 22. OSHA-Recordable Rates by Age and Job Categories Among Women, All Diagnoses Combined

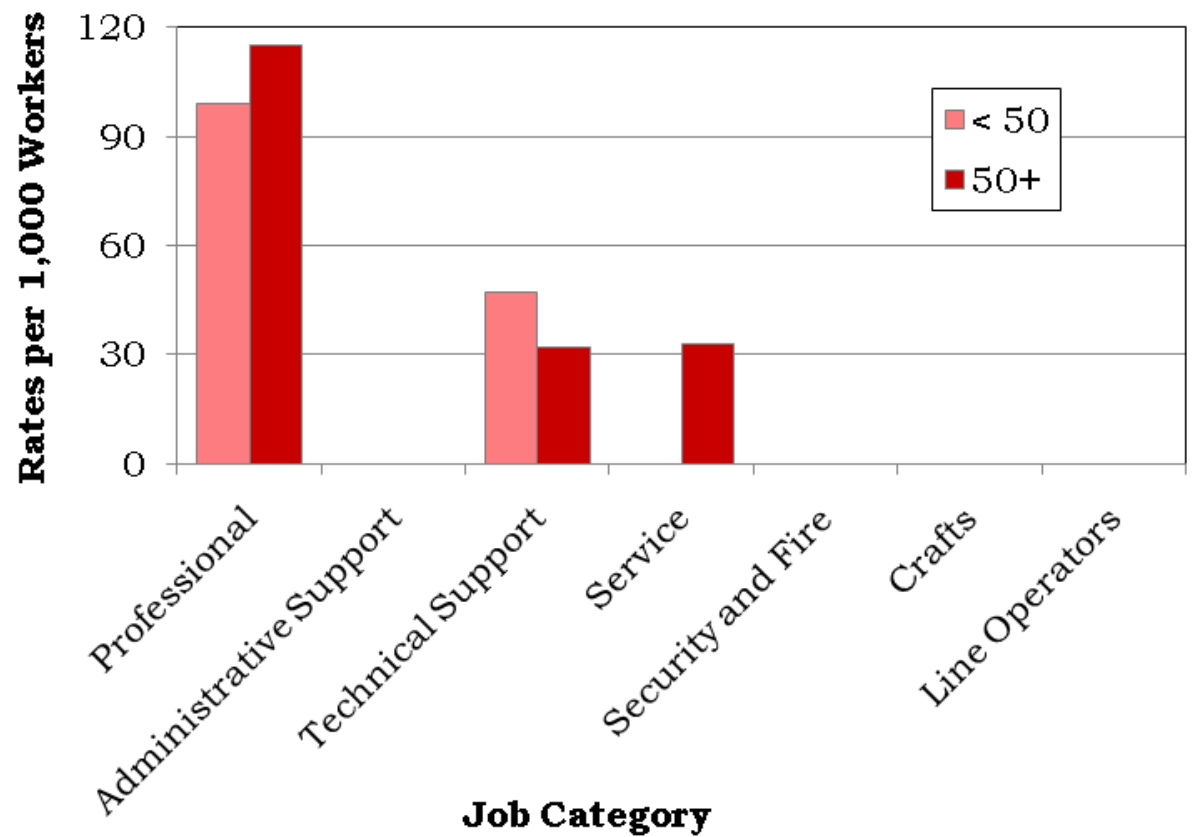

Figure 23. OSHA-Recordable Rates by Age and Job Categories Among Men, All Diagnoses Combined

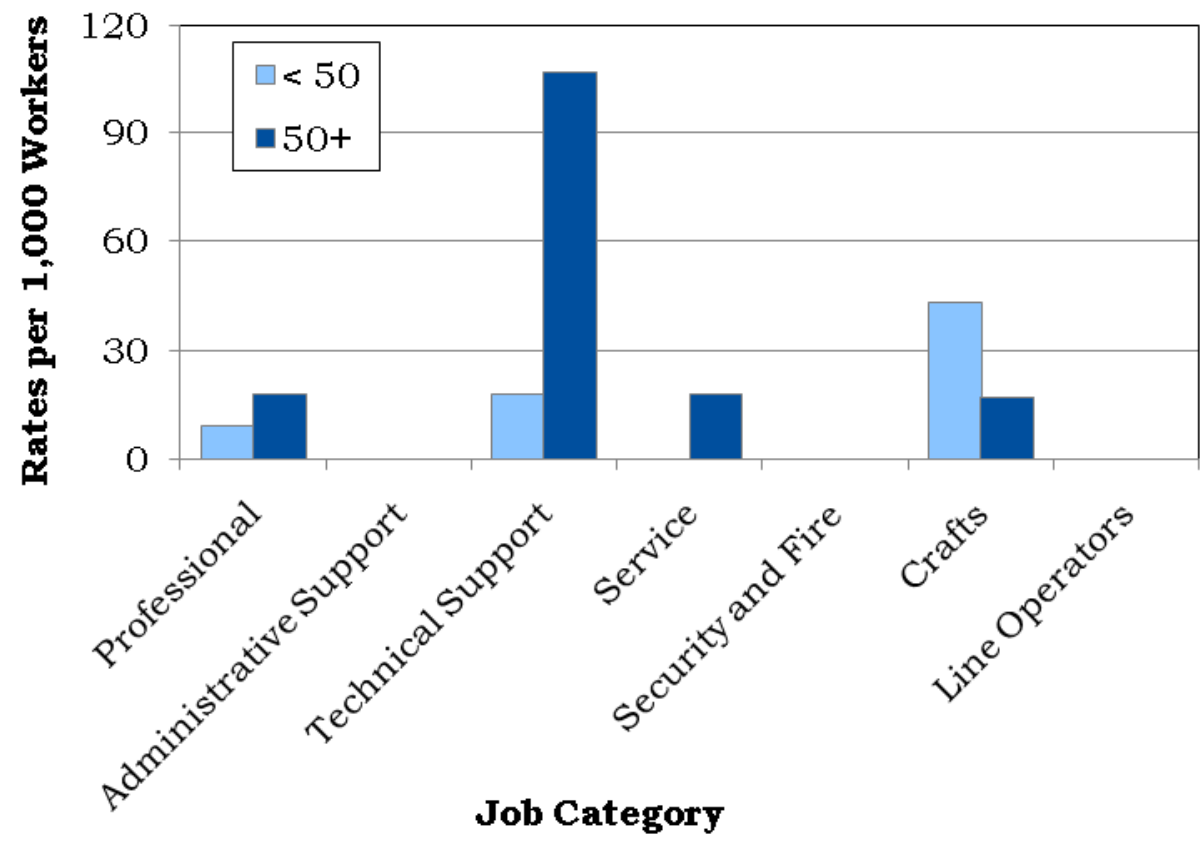




\section{Time Trends for OSHA-Recordable Events}

Figure 24. Age-Adjusted Rates for All OSHA-Recordable Diagnoses Combined Among Women and Men by Job Category from 2006 to 2010*

Professional

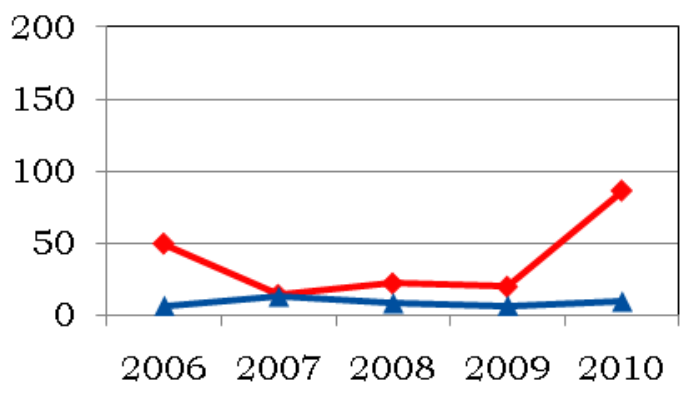

Administrative Sup port
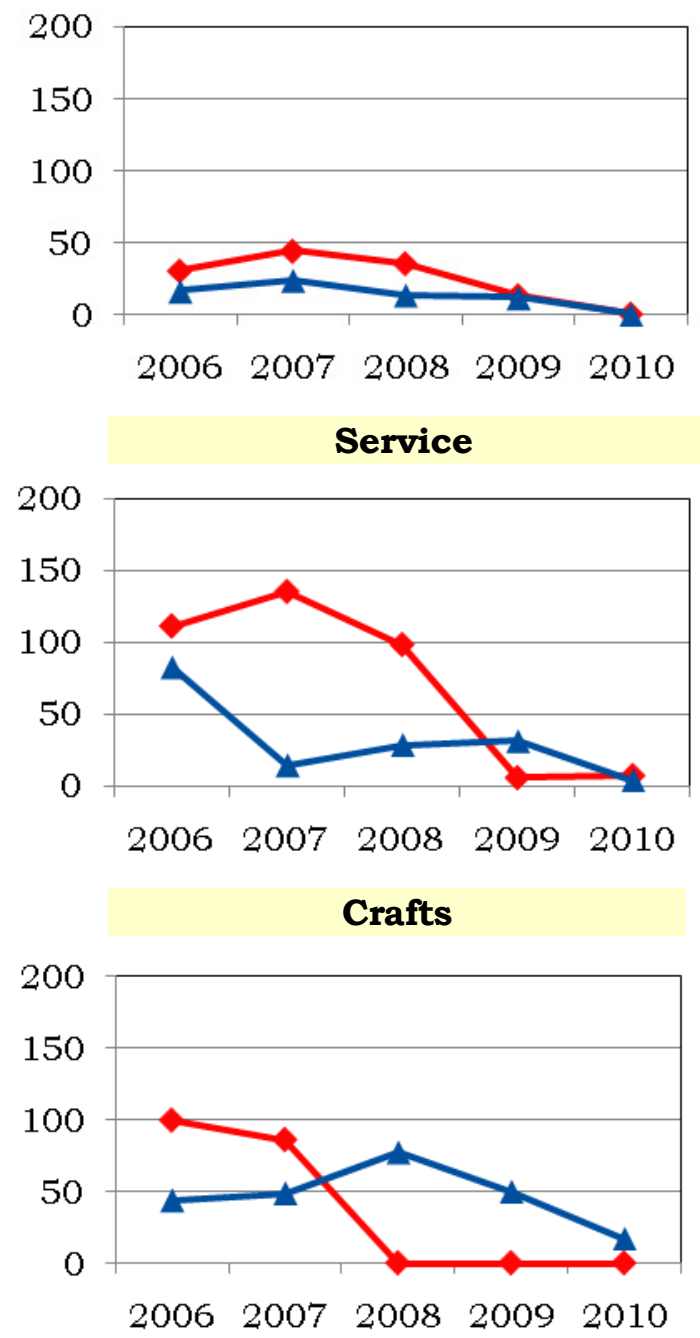

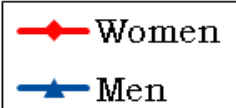

Technical Supp ort

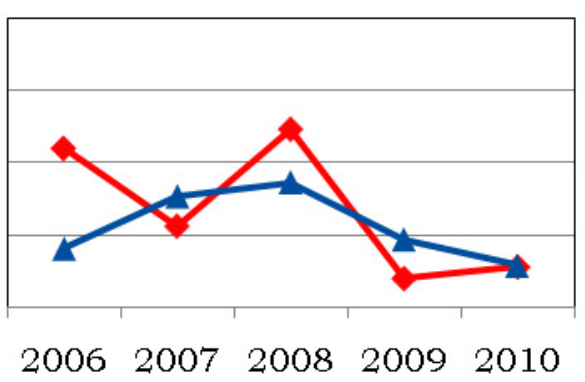

Security and Fire

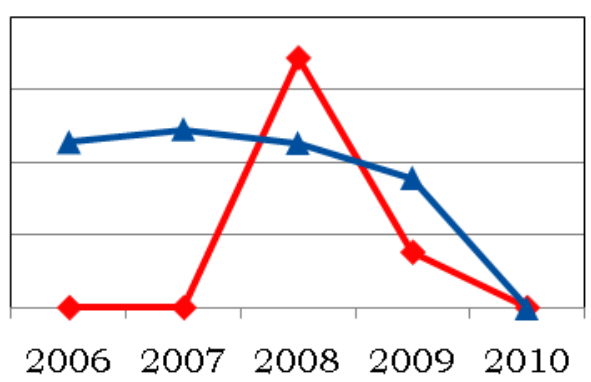

Line Operators

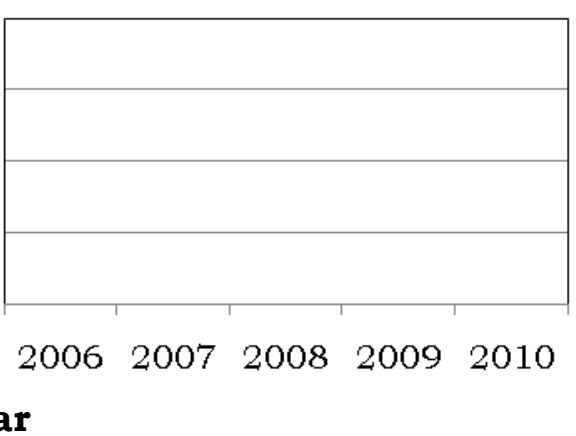

*Standardized to age distribution of 2000 U.S. population. 


\section{Appendices}


Lawrence Livermore National Laboratory 2010

Absence Data

Appendix A. Work Force by Gender, Age, and Job Category

\begin{tabular}{|c|c|c|c|c|c|c|c|c|c|c|c|}
\hline \multirow{3}{*}{ Job Category } & \multicolumn{5}{|c|}{ Women } & \multicolumn{5}{|c|}{ Men } & \multirow[b]{3}{*}{ TOTAL } \\
\hline & \multicolumn{4}{|c|}{ Age Group } & \multirow[b]{2}{*}{ TOTAL } & \multicolumn{4}{|c|}{ Age Group } & \multirow[b]{2}{*}{ TOTAL } & \\
\hline & $16-29$ & 30 - 39 & $40-49$ & $50+$ & & $16-29$ & 30 - 39 & $40-49$ & $50+$ & & \\
\hline Professional & 16 & 108 & 178 & 235 & 537 & 93 & 377 & 689 & 1,446 & 2,605 & 3,142 \\
\hline Administrative Support & 19 & 135 & 360 & 551 & 1,065 & 12 & 73 & 166 & 342 & 593 & 1,658 \\
\hline Technical Support & 3 & 43 & 39 & 63 & 148 & 13 & 61 & 154 & 234 & 462 & 610 \\
\hline Service & 2 & 7 & 23 & 60 & 92 & 3 & 21 & 58 & 112 & 194 & 286 \\
\hline Security and Fire & 2 & 2 & 3 & 10 & 17 & 25 & 84 & 70 & 56 & 235 & 252 \\
\hline Crafts & 0 & 1 & 2 & 3 & 6 & 7 & 19 & 67 & 121 & 214 & 220 \\
\hline TOTAL & 42 & 296 & 605 & 922 & 1,865 & 153 & 635 & 1,204 & 2,311 & 4,303 & 6,168 \\
\hline
\end{tabular}

Appendix B. Age Distribution of the Work Force by Gender

\begin{tabular}{|l|r|r|r|r|r|r|r|r|}
\hline \multirow{3}{*}{ Year } & \multicolumn{4}{|c|}{ Women } & \multicolumn{4}{c|}{ Men } \\
\cline { 2 - 9 } & \multicolumn{2}{|c|}{ Percent Distribution by Age Group } & \multicolumn{1}{c|}{ Percent Distribution by Age Group } \\
\cline { 2 - 9 } & $\mathbf{1 6}-\mathbf{2 9}$ & $\mathbf{3 0}-\mathbf{3 9}$ & $\mathbf{4 0 - 4 9}$ & $\mathbf{5 0}+$ & $\mathbf{1 6}-\mathbf{2 9}$ & $\mathbf{3 0}-\mathbf{3 9}$ & $\mathbf{4 0}-\mathbf{4 9}$ & $\mathbf{5 0}+$ \\
\hline $\mathbf{2 0 0 2}$ & 13.04 & 19.68 & 35.45 & 31.83 & 10.25 & 18.23 & 32.16 & 39.35 \\
\hline $\mathbf{2 0 0 3}$ & 14.40 & 16.07 & 39.17 & 30.37 & 11.31 & 15.34 & 34.82 & 38.53 \\
\hline $\mathbf{2 0 0 4}$ & 14.34 & 16.24 & 37.93 & 31.48 & 11.59 & 14.87 & 33.85 & 39.70 \\
\hline $\mathbf{2 0 0 5}$ & 12.77 & 16.87 & 33.95 & 36.41 & 10.41 & 16.04 & 28.99 & 44.56 \\
\hline $\mathbf{2 0 0 6}$ & 11.75 & 16.79 & 33.08 & 38.37 & 9.36 & 16.03 & 28.14 & 46.48 \\
\hline $\mathbf{2 0 0 7}$ & 11.70 & 15.36 & 36.85 & 36.09 & 9.81 & 14.58 & 31.34 & 44.28 \\
\hline $\mathbf{2 0 0 8}$ & 6.22 & 14.95 & 38.90 & 39.93 & 4.89 & 13.63 & 32.83 & 48.65 \\
\hline $\mathbf{2 0 0 9}$ & 3.16 & 15.54 & 34.51 & 46.78 & 3.55 & 15.15 & 27.78 & 53.53 \\
\hline $\mathbf{2 0 1 0}$ & 2.25 & 15.87 & 32.44 & 49.44 & 3.56 & 14.76 & 27.98 & 53.71 \\
\hline
\end{tabular}


Lawrence Livermore National Laboratory 2010

Absence Data

Appendix C. Total Number of Workers Who Reported at Least One Absence by Gender, Age, and Job Category*

\begin{tabular}{|c|c|c|c|c|c|c|c|c|c|c|c|}
\hline \multirow{3}{*}{ Job Category } & \multicolumn{5}{|c|}{ Women } & \multicolumn{5}{|c|}{ Men } & \multirow[b]{3}{*}{ TOTAL } \\
\hline & \multicolumn{4}{|c|}{ Age Group } & \multirow[b]{2}{*}{ TOTAL } & \multicolumn{4}{|c|}{ Age Group } & \multirow[b]{2}{*}{ TOTAL } & \\
\hline & $16-29$ & 30 - 39 & $40-49$ & $50+$ & & $16-29$ & 30 - 39 & $40-49$ & $50+$ & & \\
\hline Professional & 1 & 4 & 17 & 17 & 39 & 2 & 11 & 32 & 103 & 148 & 187 \\
\hline Administrative Support & 0 & 10 & 34 & 79 & 123 & 0 & 5 & 13 & 38 & 56 & 179 \\
\hline Technical Support & 1 & 4 & 4 & 6 & 15 & 1 & 2 & 14 & 24 & 41 & 56 \\
\hline Service & 0 & 1 & 3 & 10 & 14 & 0 & 0 & 5 & 15 & 20 & 34 \\
\hline Security and Fire & 0 & 0 & 2 & 1 & 3 & 3 & 4 & 3 & 6 & 16 & 19 \\
\hline Crafts & 0 & 0 & 1 & 0 & 1 & 0 & 2 & 2 & 10 & 14 & 15 \\
\hline TOTAL & 2 & 19 & 61 & 113 & 195 & 6 & 24 & 69 & 196 & 295 & 490 \\
\hline
\end{tabular}

*Only those job categories and gender/age combinations with at least one absence appear in this table.

Appendix D. Total Number of Absences by Gender, Age, and Job Category*

\begin{tabular}{|c|c|c|c|c|c|c|c|c|c|c|c|}
\hline \multirow{3}{*}{ Job Category } & \multicolumn{5}{|c|}{ Women } & \multicolumn{5}{|c|}{ Men } & \multirow[b]{3}{*}{ TOTAL } \\
\hline & \multicolumn{4}{|c|}{ Age Group } & \multirow[b]{2}{*}{ TOTAL } & \multicolumn{4}{|c|}{ Age Group } & \multirow[b]{2}{*}{ TOTAL } & \\
\hline & $16-29$ & 30 - 39 & $40-49$ & $50+$ & & $16-29$ & 30 - 39 & $40-49$ & $50+$ & & \\
\hline Professional & 1 & 4 & 21 & 19 & 45 & 2 & 11 & 34 & 115 & 162 & 207 \\
\hline Administrative Support & 0 & 10 & 37 & 89 & 136 & 0 & 5 & 13 & 40 & 58 & 194 \\
\hline Technical Support & 1 & 4 & 4 & 8 & 17 & 2 & 2 & 16 & 26 & 46 & 63 \\
\hline Service & 0 & 1 & 3 & 11 & 15 & 0 & 0 & 5 & 18 & 23 & 38 \\
\hline Security and Fire & 0 & 0 & 2 & 1 & 3 & 3 & 4 & 3 & 6 & 16 & 19 \\
\hline Crafts & 0 & 0 & 1 & 0 & 1 & 0 & 3 & 4 & 13 & 20 & 21 \\
\hline TOTAL & 2 & 19 & 68 & 128 & 217 & 7 & 25 & 75 & 218 & 325 & 542 \\
\hline
\end{tabular}

*Only those job categories and gender/age combinations with at least one absence appear in this table. 
Lawrence Livermore National Laboratory 2010

Absence Data

Appendix E. Distribution of the Number of Calendar Days Missed per Absence by Gender and Age*

\begin{tabular}{|c|c|c|c|c|c|c|c|c|c|c|c|}
\hline \multirow{3}{*}{$\begin{array}{c}\text { Number of } \\
\text { Calendar Days }\end{array}$} & \multicolumn{5}{|c|}{ Women } & \multicolumn{5}{|c|}{ Men } & \multirow[b]{3}{*}{ TOTAL } \\
\hline & \multicolumn{4}{|c|}{ Age Group } & \multirow[b]{2}{*}{ TOTAL } & \multicolumn{4}{|c|}{ Age Group } & \multirow[b]{2}{*}{ TOTAL } & \\
\hline & $16-29$ & $30-39$ & $40-49$ & $50+$ & & $16-29$ & $30-39$ & $40-49$ & $50+$ & & \\
\hline$<15$ & 0 & 11 & 38 & 71 & 120 & 6 & 18 & 46 & 118 & 188 & 308 \\
\hline $15-28$ & 1 & 2 & 8 & 17 & 28 & 0 & 5 & 15 & 48 & 68 & 96 \\
\hline $29-42$ & 0 & 3 & 9 & 14 & 26 & 0 & 0 & 6 & 9 & 15 & 41 \\
\hline $43-56$ & 1 & 2 & 6 & 3 & 12 & 0 & 1 & 0 & 8 & 9 & 21 \\
\hline $57-91$ & 0 & 1 & 4 & 11 & 16 & 0 & 0 & 5 & 13 & 18 & 34 \\
\hline $92-182$ & 0 & 0 & 2 & 7 & 9 & 1 & 1 & 2 & 15 & 19 & 28 \\
\hline $183+$ & 0 & 0 & 1 & 5 & 6 & 0 & 0 & 1 & 7 & 8 & 14 \\
\hline TOTAL & 2 & 19 & 68 & 128 & 217 & 7 & 25 & 75 & 218 & 325 & 542 \\
\hline
\end{tabular}

*Only those gender/age combinations with at least one absence appear in this table. 
Lawrence Livermore National Laboratory 2010

Absence Data

Appendix F. Distribution of the Number of Calendar Days Missed per Absence by Gender and Job Category*

Women

\begin{tabular}{|c|c|c|c|c|c|c|c|}
\hline \multirow{2}{*}{$\begin{array}{c}\text { Number of } \\
\text { Calendar Days }\end{array}$} & \multicolumn{6}{|c|}{ Job Category } & \multirow[b]{2}{*}{ TOTAL } \\
\hline & Professional & $\begin{array}{c}\text { Administrative } \\
\text { Support }\end{array}$ & Technical Support & Service & Security and Fire & Crafts & \\
\hline$<15$ & 27 & 71 & 11 & 9 & 1 & 1 & 120 \\
\hline $15-28$ & 6 & 17 & 3 & 1 & 1 & 0 & 28 \\
\hline $29-42$ & 4 & 21 & 0 & 1 & 0 & 0 & 26 \\
\hline $43-56$ & 5 & 4 & 1 & 2 & 0 & 0 & 12 \\
\hline $57-91$ & 2 & 12 & 1 & 1 & 0 & 0 & 16 \\
\hline $92-182$ & 1 & 6 & 0 & 1 & 1 & 0 & 9 \\
\hline $183+$ & 0 & 5 & 1 & 0 & 0 & 0 & 6 \\
\hline TOTAL & 45 & 136 & 17 & 15 & 3 & 1 & 217 \\
\hline
\end{tabular}

Men

\begin{tabular}{|c|c|c|c|c|c|c|c|}
\hline \multirow{2}{*}{$\begin{array}{c}\text { Number of } \\
\text { Calendar Days }\end{array}$} & \multicolumn{6}{|c|}{ Job Category } & \multirow[b]{2}{*}{ TOTAL } \\
\hline & Professional & $\begin{array}{c}\text { Administrative } \\
\text { Support }\end{array}$ & Technical Support & Service & Security and Fire & Crafts & \\
\hline$<15$ & 101 & 32 & 25 & 14 & 9 & 7 & 188 \\
\hline $15-28$ & 36 & 13 & 8 & 5 & 2 & 4 & 68 \\
\hline $29-42$ & 7 & 2 & 2 & 1 & 2 & 1 & 15 \\
\hline $43-56$ & 3 & 4 & 1 & 1 & 0 & 0 & 9 \\
\hline $57-91$ & 7 & 1 & 5 & 1 & 0 & 4 & 18 \\
\hline $92-182$ & 7 & 4 & 4 & 0 & 3 & 1 & 19 \\
\hline $183+$ & 1 & 2 & 1 & 1 & 0 & 3 & 8 \\
\hline TOTAL & 162 & 58 & 46 & 23 & 16 & 20 & 325 \\
\hline
\end{tabular}

*Only those gender/job category combinations with at least one absence appear in this table. 


\section{Lawrence Livermore National Laboratory 2010}

Absence Data

Appendix G. Number of Diagnoses in Each Diagnostic Category by Gender and Age*

\begin{tabular}{|c|c|c|c|c|c|c|}
\hline & & \multicolumn{5}{|c|}{ Women } \\
\hline & & \multicolumn{4}{|c|}{ Age Group } & \multirow[b]{2}{*}{ TOTAL } \\
\hline & & $16-29$ & $30-39$ & $40-49$ & $50+$ & \\
\hline Diagnostic Category & ICD-9-CM Code & \multirow[b]{2}{*}{0} & \multirow[b]{2}{*}{0} & \multirow[b]{2}{*}{0} & \multirow[b]{2}{*}{8} & \multirow[b]{2}{*}{8} \\
\hline INFECTIOUS \& PARASITIC DISEASES (DIS) & 001-139 & & & & & \\
\hline -Intestinal Infectious Dis & 001-009 & 0 & 0 & 0 & 5 & 5 \\
\hline -Other Bacterial Dis & 030-041 & 0 & 0 & 0 & 0 & 0 \\
\hline -Polio \& Other Viral CNS Dis & 045-049 & 0 & 0 & 0 & 1 & 1 \\
\hline -Viral Dis with Exanthem & 050-059 & 0 & 0 & 0 & 1 & 1 \\
\hline -Other Viral Dis \& Chlamydiae & 070-079 & 0 & 0 & 0 & 1 & 1 \\
\hline MALIGNANT NEOPLASMS & $\begin{array}{l}140-208, \\
209.0-209.3 \\
230-234\end{array}$ & 0 & 0 & 2 & 3 & 5 \\
\hline -Digestive \& Peritoneal & $150-159$ & 0 & 0 & 0 & 0 & 0 \\
\hline -Respiratory \& Intrathoracic & $160-165$ & 0 & 0 & 0 & 0 & 0 \\
\hline -Breast & 174-175 & 0 & 0 & 2 & 3 & 5 \\
\hline -Genitourinary & 179-189 & 0 & 0 & 0 & 0 & 0 \\
\hline -Endocrine & $\begin{array}{l}\text { 193-194, } \\
209.0-209.3\end{array}$ & 0 & 0 & 0 & 0 & 0 \\
\hline -Other \& Unspecified Sites & 195-199, 209.7 & 0 & 0 & 0 & 0 & 0 \\
\hline -Lymphatic \& Hematopoietic & $200-208$ & 0 & 0 & 0 & 0 & 0 \\
\hline BENIGN \& UNCERTAIN NEOPLASMS & $\begin{array}{l}209.4-209.6 \\
210-229,235-239\end{array}$ & 0 & 1 & 2 & 2 & 5 \\
\hline ENDOCRINE/METABOLIC/IMMUNITY & $240-279$ & 0 & 0 & 0 & 3 & 3 \\
\hline -Other Endocrine Gland Dis & 249-259 & 0 & 0 & 0 & 1 & 1 \\
\hline -Other Metabolic \& Immunity Disorders & $270-279$ & 0 & 0 & 0 & 2 & 2 \\
\hline BLOOD \& BLOOD-FORMING ORGANS & $280-289$ & 0 & 0 & 0 & 0 & 0 \\
\hline MENTAL DISORDERS & $290-319$ & 0 & 0 & 1 & 0 & 1 \\
\hline -Non-Psychotic Disorders & $300-302,306-316$ & 0 & 0 & 1 & 0 & 1 \\
\hline -Alcohol Dependence & 303 & 0 & 0 & 0 & 0 & 0 \\
\hline -Drug Dependence & $304-305$ & 0 & 0 & 0 & 0 & 0 \\
\hline NERVOUS SYSTEM (NS) \& SENSE ORGANS & 320-389 & 1 & 1 & 1 & 7 & 10 \\
\hline -Hereditary/Degenerative Central NS Dis & 330-337 & 0 & 0 & 0 & 0 & 0 \\
\hline -Other Disorders of Central NS & $340-349$ & 1 & 1 & 1 & 0 & 3 \\
\hline -Disorders of Peripheral NS & $350-359$ & 0 & 0 & 0 & 5 & 5 \\
\hline -Disorders of Eye & $360-379$ & 0 & 0 & 0 & 2 & 2 \\
\hline -Diseases of Ear \& Mastoid & $380-389$ & 0 & 0 & 0 & 0 & 0 \\
\hline CIRCULATORY SYSTEM & $390-459$ & 0 & 0 & 1 & 8 & 9 \\
\hline -Hypertensive Dis & $401-405$ & 0 & 0 & 0 & 1 & 1 \\
\hline -Ischemic Heart Dis & $410-414$ & 0 & 0 & 0 & 0 & 0 \\
\hline -Dis of Pulmonary Circulation & $415-417$ & 0 & 0 & 0 & 1 & 1 \\
\hline -Other Heart Dis & $420-429$ & 0 & 0 & 0 & 3 & 3 \\
\hline
\end{tabular}

(Continued)

*Only those diagnostic categories and gender/age combinations with at least one occurrence appear in this table. 


\section{Lawrence Livermore National Laboratory 2010}

Absence Data

Appendix G. Number of Diagnoses in Each Diagnostic Category by Gender and Age*

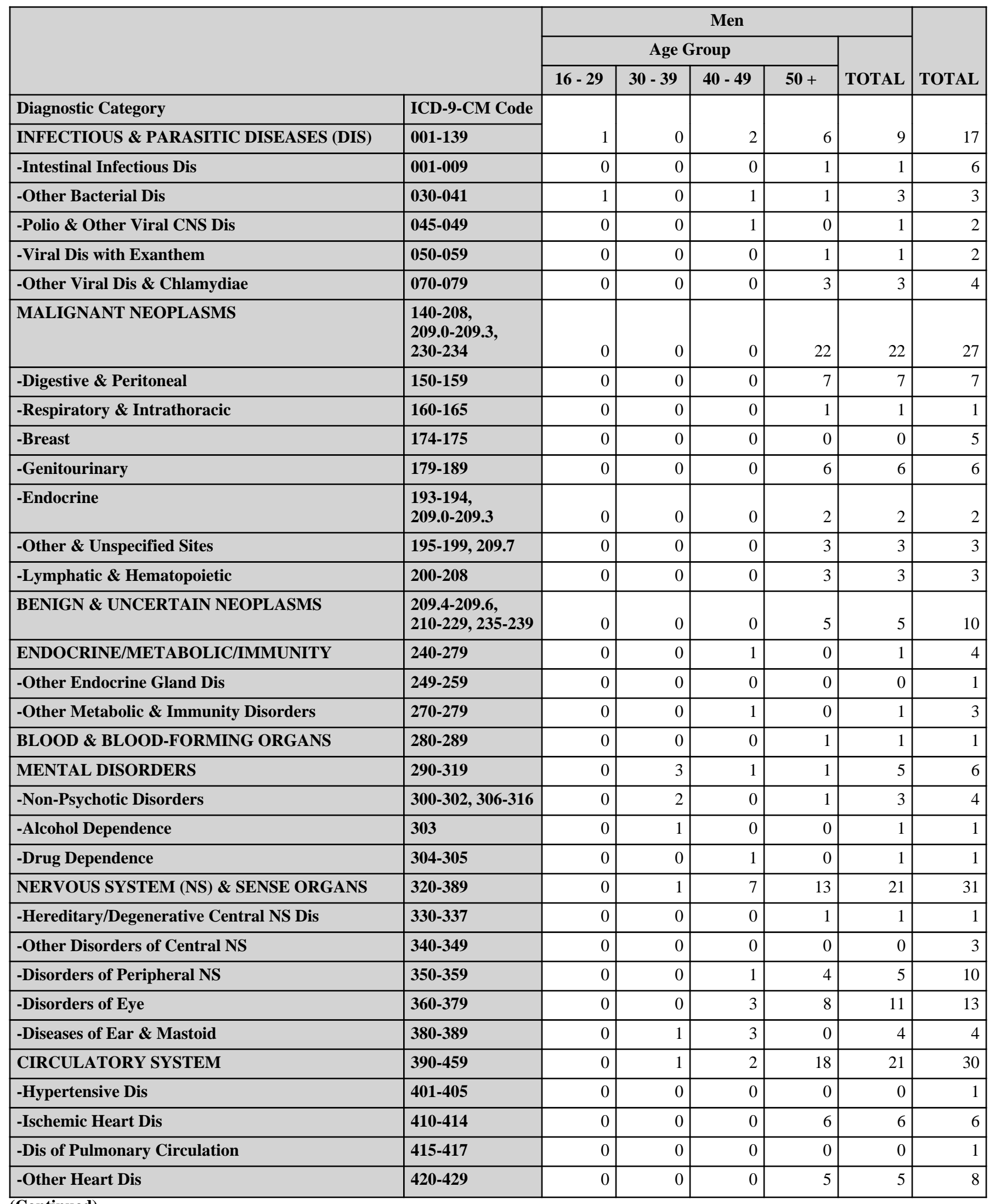

(Continued)

*Only those diagnostic categories and gender/age combinations with at least one occurrence appear in this table. 


\section{Lawrence Livermore National Laboratory 2010}

Absence Data

Appendix G. Number of Diagnoses in Each Diagnostic Category by Gender and Age*

\begin{tabular}{|c|c|c|c|c|c|c|}
\hline & & \multicolumn{5}{|c|}{ Women } \\
\hline & & \multicolumn{4}{|c|}{ Age Group } & \multirow[b]{2}{*}{ TOTAL } \\
\hline & & $16-29$ & 30 - 39 & $40-49$ & $50+$ & \\
\hline Diagnostic Category & ICD-9-CM Code & \multirow[b]{2}{*}{0} & \multirow[b]{2}{*}{0} & \multirow[b]{2}{*}{0} & \multirow[b]{2}{*}{1} & \multirow[b]{2}{*}{1} \\
\hline -Cerebrovascular Dis & $430-438$ & & & & & \\
\hline -Dis of Arteries \& Capillaries & $440-449$ & 0 & 0 & 0 & 0 & 0 \\
\hline -Dis of Veins, Lymphatics, Other & $451-459$ & 0 & 0 & 1 & 2 & 3 \\
\hline RESPIRATORY SYSTEM & $460-519$ & 0 & 5 & 15 & 25 & 45 \\
\hline -Acute Respiratory Infections & $460-466$ & 0 & 2 & 8 & 6 & 16 \\
\hline -Other Dis Upper Respiratory Tract & $470-478$ & 0 & 1 & 1 & 4 & 6 \\
\hline -Pneumonia \& Influenza & $480-488$ & 0 & 2 & 5 & 12 & 19 \\
\hline -Chronic Obstructive Dis & $490-496$ & 0 & 0 & 1 & 2 & 3 \\
\hline -Other Respiratory Dis & $510-519$ & 0 & 0 & 0 & 1 & 1 \\
\hline DIGESTIVE SYSTEM & $520-579$ & 0 & 3 & 6 & 16 & 25 \\
\hline -Oral Cavity, Saliva Glands, Jaw & $520-529$ & 0 & 1 & 0 & 2 & 3 \\
\hline -Esophagus, Stomach, Duodenum & $530-538$ & 0 & 0 & 0 & 2 & 2 \\
\hline -Appendicitis & $540-543$ & 0 & 0 & 3 & 1 & 4 \\
\hline -Hernias & $550-553$ & 0 & 0 & 0 & 2 & 2 \\
\hline -Enteritis, Colitis & $555-558$ & 0 & 0 & 0 & 1 & 1 \\
\hline -Other Intestinal Dis & $560-569$ & 0 & 0 & 2 & 3 & 5 \\
\hline -Other Digestive Dis & $570-579$ & 0 & 2 & 1 & 5 & 8 \\
\hline GENITOURINARY SYSTEM & $580-629$ & 0 & 3 & 11 & 6 & 20 \\
\hline -Other Urinary Dis & $590-599$ & 0 & 0 & 1 & 3 & 4 \\
\hline -Male Genital Organ Dis & $600-608$ & 0 & 0 & 0 & 0 & 0 \\
\hline -Breast Disorders & 610-612 & 0 & 0 & 1 & 0 & 1 \\
\hline -Other Female Disorders & 617-629 & 0 & 3 & 9 & 3 & 15 \\
\hline PREGNANCY \& CHILDBIRTH & $630-679$ & 0 & 1 & 0 & 0 & 1 \\
\hline -Pregnancy with Abortive Outcome & $634-639$ & 0 & 1 & 0 & 0 & 1 \\
\hline SKIN AND SUBCUTANEOUS TISSUE & 680-709 & 0 & 0 & 0 & 2 & 2 \\
\hline -Infections & $680-686$ & 0 & 0 & 0 & 1 & 1 \\
\hline -Other & 700-709 & 0 & 0 & 0 & 1 & 1 \\
\hline $\begin{array}{l}\text { MUSCULOSKELETAL \& CONNECTIVE } \\
\text { TISSUE }\end{array}$ & $710-739$ & 0 & 1 & 20 & 31 & 52 \\
\hline -Arthropathies & 710-719 & 0 & 0 & 6 & 12 & 18 \\
\hline -Dorsopathies & 720-724 & 0 & 1 & 6 & 12 & 19 \\
\hline -Rheumatism, Excluding Back & 725-729 & 0 & 0 & 7 & 6 & 13 \\
\hline -Other Dis \& Acquired Deformities & 730-739 & 0 & 0 & 1 & 1 & 2 \\
\hline $\begin{array}{l}\text { SYMPTOMS, SIGNS, \& ILL-DEFINED } \\
\text { CONDITIONS }\end{array}$ & 780-799 & 0 & 2 & 7 & 19 & 28 \\
\hline -Symptoms & 780-789 & 0 & 2 & 7 & 19 & 28 \\
\hline INJURY \& POISONING & $800-999$ & 1 & 2 & 7 & 15 & 25 \\
\hline
\end{tabular}

(Continued)

*Only those diagnostic categories and gender/age combinations with at least one occurrence appear in this table. 


\section{Lawrence Livermore National Laboratory 2010}

\section{Absence Data}

Appendix G. Number of Diagnoses in Each Diagnostic Category by Gender and Age*

\begin{tabular}{|c|c|c|c|c|c|c|c|}
\hline & & \multicolumn{5}{|c|}{ Men } & \multirow[b]{3}{*}{ TOTAL } \\
\hline & & \multicolumn{4}{|c|}{ Age Group } & \multirow[b]{2}{*}{ TOTAL } & \\
\hline & & $16-29$ & 30 - 39 & $40-49$ & $50+$ & & \\
\hline -Cerebrovascular Dis & $430-438$ & 0 & 0 & 1 & 3 & 4 & 5 \\
\hline -Dis of Arteries \& Capillaries & $440-449$ & 0 & 0 & 1 & 2 & 3 & 3 \\
\hline RESPIRATORY SYSTEM & $460-519$ & 3 & 5 & 20 & 36 & 64 & 109 \\
\hline -Acute Respiratory Infections & $460-466$ & 0 & 3 & 7 & 15 & 25 & 41 \\
\hline -Other Dis Upper Respiratory Tract & $470-478$ & 0 & 1 & 2 & 5 & 8 & 14 \\
\hline -Pneumonia \& Influenza & $480-488$ & 3 & 1 & 9 & 14 & 27 & 46 \\
\hline -Oral Cavity, Saliva Glands, Jaw & $520-529$ & 0 & 1 & 1 & 1 & 3 & 6 \\
\hline -Esophagus, Stomach, Duodenum & $530-538$ & 0 & 0 & 1 & 3 & 4 & 6 \\
\hline -Appendicitis & $540-543$ & 0 & 0 & 0 & 4 & 4 & 8 \\
\hline -Hernias & $550-553$ & 0 & 2 & 1 & 14 & 17 & 19 \\
\hline -Enteritis, Colitis & $555-558$ & 0 & 0 & 0 & 0 & 0 & 1 \\
\hline -Other Intestinal Dis & $560-569$ & 0 & 1 & 0 & 3 & 4 & 9 \\
\hline -Other Digestive Dis & $570-579$ & 0 & 0 & 4 & 5 & 9 & 17 \\
\hline GENITOURINARY SYSTEM & $580-629$ & 0 & 0 & 2 & 8 & 10 & 30 \\
\hline -Pregnancy with Abortive Outcome & 634-639 & 0 & 0 & 0 & 0 & 0 & 1 \\
\hline SKIN AND SUBCUTANEOUS TISSUE & $680-709$ & 0 & 0 & 1 & 2 & 3 & 5 \\
\hline -Infections & $680-686$ & 0 & 0 & 1 & 0 & 1 & 2 \\
\hline -Other & 700-709 & 0 & 0 & 0 & 2 & 2 & 3 \\
\hline $\begin{array}{l}\text { MUSCULOSKELETAL \& CONNECTIVE } \\
\text { TISSUE }\end{array}$ & $710-739$ & 1 & 3 & 14 & 51 & 69 & 121 \\
\hline -Arthropathies & 710-719 & 1 & 1 & 6 & 26 & 34 & 52 \\
\hline -Dorsopathies & $720-724$ & 0 & 1 & 7 & 15 & 23 & 42 \\
\hline -Rheumatism, Excluding Back & 725-729 & 0 & 1 & 1 & 9 & 11 & 24 \\
\hline -Other Dis \& Acquired Deformities & $730-739$ & 0 & 0 & 0 & 1 & 1 & 3 \\
\hline $\begin{array}{l}\text { SYMPTOMS, SIGNS, \& ILL-DEFINED } \\
\text { CONDITIONS }\end{array}$ & $780-799$ & 0 & 2 & 8 & 17 & 27 & 55 \\
\hline -Symptoms & 780-789 & 0 & 2 & 8 & 17 & 27 & 55 \\
\hline INJURY \& POISONING & $800-999$ & 3 & 6 & 18 & 32 & 59 & 84 \\
\hline
\end{tabular}

(Continued)

*Only those diagnostic categories and gender/age combinations with at least one occurrence appear in this table. 


\section{Lawrence Livermore National Laboratory 2010}

Absence Data

Appendix G. Number of Diagnoses in Each Diagnostic Category by Gender and Age*

\begin{tabular}{|c|c|c|c|c|c|c|}
\hline & & \multicolumn{5}{|c|}{ Women } \\
\hline & & \multicolumn{4}{|c|}{ Age Group } & \multirow[b]{2}{*}{ TOTAL } \\
\hline & & $16-29$ & $30-39$ & $40-49$ & $50+$ & \\
\hline Diagnostic Category & ICD-9-CM Code & \multirow[b]{2}{*}{0} & \multirow[b]{2}{*}{0} & \multirow[b]{2}{*}{1} & \multirow[b]{2}{*}{2} & \multirow[b]{2}{*}{3} \\
\hline -Fracture - Upper Limb & $810-819$ & & & & & \\
\hline -Fracture - Lower Limb & $820-829$ & 0 & 1 & 0 & 4 & 5 \\
\hline -Dislocation & 830-839 & 0 & 0 & 2 & 3 & 5 \\
\hline -Sprains \& Strains - Back & $846-847$ & 0 & 0 & 0 & 0 & 0 \\
\hline -Sprains \& Strains - Other & $840-845,848$ & 0 & 0 & 3 & 4 & 7 \\
\hline -Intracranial Injury & \begin{tabular}{|l|}
$850-854$ \\
\end{tabular} & 0 & 0 & 1 & 0 & 1 \\
\hline -Open Wound - Head, Neck, Trunk & $870-879$ & 0 & 0 & 0 & 0 & 0 \\
\hline -Open Wound - Lower Limb & \begin{tabular}{|l|}
$890-897$ \\
\end{tabular} & 0 & 0 & 0 & 1 & 1 \\
\hline -Superficial Injury & 910-919 & 0 & 0 & 0 & 0 & 0 \\
\hline -Contusion & $920-924$ & 0 & 0 & 0 & 1 & 1 \\
\hline -Complications \& Unspecified Injuries & 958-959 & 1 & 1 & 0 & 0 & 2 \\
\hline $\begin{array}{l}\text { HEALTH STATUS/HEALTH SERVICE } \\
\text { CONTACT }\end{array}$ & V01-V89 & 0 & 0 & 2 & 3 & 5 \\
\hline -Personal \& Family History & V10-V19 & 0 & 0 & 0 & 0 & 0 \\
\hline -Health Services Reproduction/Development & V20-V29 & 0 & 0 & 1 & 0 & 1 \\
\hline -Specific Procedures/Aftercare & V50-V59 & 0 & 0 & 1 & 3 & 4 \\
\hline -Examination \& Investigation & V70-V82 & 0 & 0 & 0 & 0 & 0 \\
\hline
\end{tabular}

*Only those diagnostic categories and gender/age combinations with at least one occurrence appear in this table. 


\section{Lawrence Livermore National Laboratory 2010}

Absence Data

Appendix G. Number of Diagnoses in Each Diagnostic Category by Gender and Age*

\begin{tabular}{|c|c|c|c|c|c|c|c|}
\hline & & \multicolumn{5}{|c|}{ Men } & \multirow[b]{3}{*}{ TOTAL } \\
\hline & & \multicolumn{4}{|c|}{ Age Group } & \multirow[b]{2}{*}{ TOTAL } & \\
\hline & & $16-29$ & $30-39$ & $40-49$ & $50+$ & & \\
\hline -Fracture - Upper Limb & 810-819 & 0 & 1 & 2 & 2 & 5 & 8 \\
\hline -Fracture - Lower Limb & 820-829 & 0 & 0 & 2 & 4 & 6 & 11 \\
\hline -Sprains \& Strains - Back & 846-847 & 0 & 0 & 2 & 3 & 5 & 5 \\
\hline -Sprains \& Strains - Other & $840-845,848$ & 3 & 4 & 9 & 11 & 27 & 34 \\
\hline -Intracranial Injury & $850-854$ & 0 & 0 & 0 & 0 & 0 & 1 \\
\hline -Open Wound - Head, Neck, Trunk & 870-879 & 0 & 0 & 0 & 1 & 1 & 1 \\
\hline -Complications \& Unspecified Injuries & 958-959 & 0 & 0 & 1 & 2 & 3 & 5 \\
\hline $\begin{array}{l}\text { HEALTH STATUS/HEALTH SERVICE } \\
\text { CONTACT }\end{array}$ & V01-V89 & 0 & 0 & 4 & 2 & 6 & 11 \\
\hline -Personal \& Family History & V10-V19 & 0 & 0 & 1 & 0 & 1 & 1 \\
\hline -Health Services Reproduction/Development & V20-V29 & 0 & 0 & 1 & 1 & 2 & 3 \\
\hline -Specific Procedures/Aftercare & V50-V59 & 0 & 0 & 0 & 0 & 0 & 4 \\
\hline -Examination \& Investigation & V70-V82 & 0 & 0 & 2 & 1 & 3 & 3 \\
\hline
\end{tabular}

\begin{tabular}{|c|c|c|c|c|c|c|c|c|c|c|c|}
\hline & \multicolumn{5}{|c|}{ Women } & \multicolumn{5}{|c|}{ Men } & \multirow[b]{3}{*}{ TOTAL } \\
\hline & \multicolumn{4}{|c|}{ Age Group } & \multirow[b]{2}{*}{ TOTAL } & \multicolumn{4}{|c|}{ Age Group } & \multirow[b]{2}{*}{ TOTAL } & \\
\hline & $16-29$ & 30 - 39 & $40-49$ & $50+$ & & $16-29$ & 30 - 39 & $40-49$ & $50+$ & & \\
\hline \multicolumn{12}{|l|}{ Diagnostic Category } \\
\hline Total & 2 & 19 & 75 & 148 & 244 & 8 & 25 & 87 & 244 & 364 & 608 \\
\hline
\end{tabular}

*Only those diagnostic categories and gender/age combinations with at least one occurrence appear in this table. 
Lawrence Livermore National Laboratory 2010

Absence Data

Appendix H. Total Number of Calendar Days Absent in Each Diagnostic Category by Gender and Age*

\begin{tabular}{|c|c|c|c|c|c|c|}
\hline & & \multicolumn{5}{|c|}{ Women } \\
\hline & & \multicolumn{4}{|c|}{ Age Group } & \multirow[b]{2}{*}{ TOTAL } \\
\hline & & $16-29$ & $30-39$ & $40-49$ & $50+$ & \\
\hline Diagnostic Category & ICD-9-CM Code & \multirow[b]{2}{*}{0} & \multirow[b]{2}{*}{0} & \multirow[b]{2}{*}{0} & \multirow[b]{2}{*}{91} & \multirow[b]{2}{*}{91} \\
\hline INFECTIOUS \& PARASITIC DISEASES (DIS) & 001-139 & & & & & \\
\hline MALIGNANT NEOPLASMS & $\begin{array}{l}140-208, \\
209.0-209.3 \\
230-234\end{array}$ & 0 & 0 & 10 & 86 & 96 \\
\hline BENIGN \& UNCERTAIN NEOPLASMS & $\begin{array}{l}209.4-209.6 \\
210-229,235-239\end{array}$ & 0 & 9 & 56 & 114 & 179 \\
\hline ENDOCRINE/METABOLIC/IMMUNITY & $240-279$ & 0 & 0 & 0 & 77 & 77 \\
\hline BLOOD \& BLOOD-FORMING ORGANS & $280-289$ & 0 & 0 & 0 & 0 & 0 \\
\hline MENTAL DISORDERS & 290-319 & 0 & 0 & 24 & 0 & 24 \\
\hline NERVOUS SYSTEM (NS) \& SENSE ORGANS & $320-389$ & 43 & 9 & 45 & 194 & 291 \\
\hline CIRCULATORY SYSTEM & $390-459$ & 0 & 0 & 23 & 222 & 245 \\
\hline RESPIRATORY SYSTEM & $460-519$ & 0 & 29 & 556 & 357 & 942 \\
\hline DIGESTIVE SYSTEM & $520-579$ & 0 & 50 & 78 & 363 & 491 \\
\hline GENITOURINARY SYSTEM & $580-629$ & 0 & 95 & 393 & 231 & 719 \\
\hline PREGNANCY \& CHILDBIRTH & $630-679$ & 0 & 12 & 0 & 0 & 12 \\
\hline SKIN AND SUBCUTANEOUS TISSUE & 680-709 & 0 & 0 & 0 & 10 & 10 \\
\hline $\begin{array}{l}\text { MUSCULOSKELETAL \& CONNECTIVE } \\
\text { TISSUE }\end{array}$ & $710-739$ & 0 & 59 & 593 & 1,876 & 2,528 \\
\hline $\begin{array}{l}\text { SYMPTOMS, SIGNS, \& ILL-DEFINED } \\
\text { CONDITIONS }\end{array}$ & $780-799$ & 0 & 61 & 527 & 498 & 1,086 \\
\hline INJURY \& POISONING & $800-999$ & 18 & 45 & 220 & 486 & 769 \\
\hline
\end{tabular}

\footnotetext{
*Absences with >1 ICD-9-CM code in the same diagnostic category were counted only once. Only those diagnostic categories and gender/age combinations with at least one occurrence appear in this table.
} 
Lawrence Livermore National Laboratory 2010

Absence Data

Appendix H. Total Number of Calendar Days Absent in Each Diagnostic Category by Gender and Age*

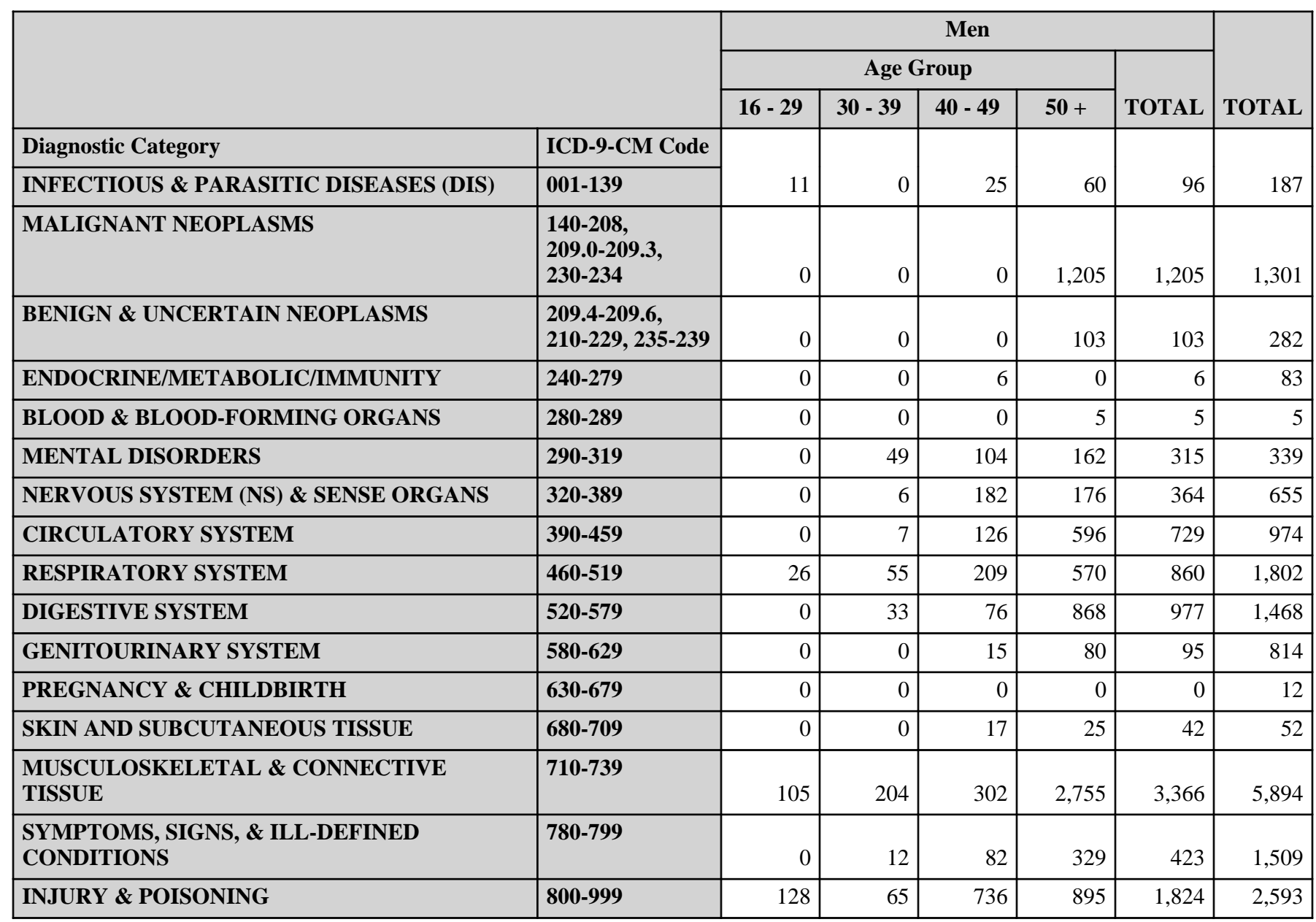

\footnotetext{
*Absences with >1 ICD-9-CM code in the same diagnostic category were counted only once. Only those diagnostic categories and gender/age combinations with at least one occurrence appear in this table.
} 


\section{Lawrence Livermore National Laboratory 2010}

Absence Data

Appendix I. Number of Diagnoses in Each Diagnostic Category by Gender and Job Category*

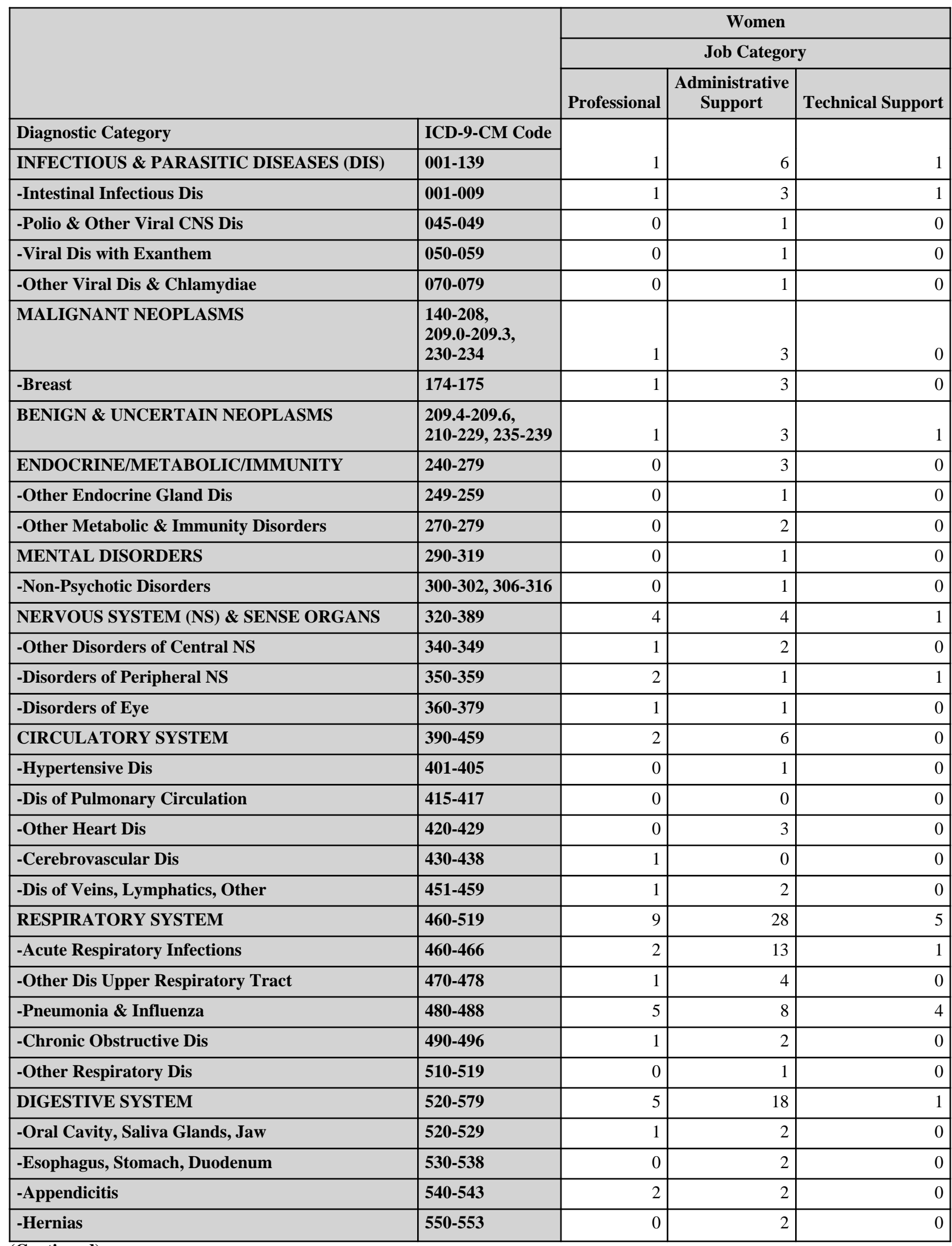

(Continued)

*Only those diagnostic categories and gender/job category combinations with at least one occurrence appear in this table. 


\section{Lawrence Livermore National Laboratory 2010}

Absence Data

Appendix I. Number of Diagnoses in Each Diagnostic Category by Gender and Job Category*

\begin{tabular}{|c|c|c|c|c|c|}
\hline & & \multicolumn{4}{|c|}{ Women } \\
\hline & & \multicolumn{3}{|c|}{ Job Category } & \multirow[b]{2}{*}{ TOTAL } \\
\hline & & Service & Security and Fire & Crafts & \\
\hline Diagnostic Category & ICD-9-CM Code & \multirow[b]{2}{*}{0} & \multirow[b]{2}{*}{0} & \multirow[b]{2}{*}{0} & \multirow[b]{2}{*}{8} \\
\hline INFECTIOUS \& PARASITIC DISEASES (DIS) & 001-139 & & & & \\
\hline -Intestinal Infectious Dis & 001-009 & 0 & 0 & 0 & 5 \\
\hline -Polio \& Other Viral CNS Dis & 045-049 & 0 & 0 & 0 & 1 \\
\hline -Viral Dis with Exanthem & $050-059$ & 0 & 0 & 0 & 1 \\
\hline -Other Viral Dis \& Chlamydiae & 070-079 & 0 & 0 & 0 & 1 \\
\hline MALIGNANT NEOPLASMS & $\begin{array}{l}140-208, \\
209.0-209.3 \\
230-234\end{array}$ & 1 & 0 & 0 & 5 \\
\hline -Breast & $174-175$ & 1 & 0 & 0 & 5 \\
\hline BENIGN \& UNCERTAIN NEOPLASMS & $\begin{array}{l}209.4-209.6 \\
210-229,235-239\end{array}$ & 0 & 0 & 0 & 5 \\
\hline ENDOCRINE/METABOLIC/IMMUNITY & $240-279$ & 0 & 0 & 0 & 3 \\
\hline -Other Endocrine Gland Dis & 249-259 & 0 & 0 & 0 & 1 \\
\hline -Other Metabolic \& Immunity Disorders & 270-279 & 0 & 0 & 0 & 2 \\
\hline MENTAL DISORDERS & $290-319$ & 0 & 0 & 0 & 1 \\
\hline -Non-Psychotic Disorders & $300-302,306-316$ & 0 & 0 & 0 & 1 \\
\hline NERVOUS SYSTEM (NS) \& SENSE ORGANS & $320-389$ & 1 & 0 & 0 & 10 \\
\hline -Other Disorders of Central NS & $340-349$ & 0 & 0 & 0 & 3 \\
\hline -Disorders of Peripheral NS & $350-359$ & 1 & 0 & 0 & 5 \\
\hline -Disorders of Eye & $360-379$ & 0 & 0 & 0 & 2 \\
\hline CIRCULATORY SYSTEM & $390-459$ & 1 & 0 & 0 & 9 \\
\hline -Hypertensive Dis & $401-405$ & 0 & 0 & 0 & 1 \\
\hline -Dis of Pulmonary Circulation & $415-417$ & 1 & 0 & 0 & 1 \\
\hline -Other Heart Dis & $420-429$ & 0 & 0 & 0 & 3 \\
\hline -Cerebrovascular Dis & $430-438$ & 0 & 0 & 0 & 1 \\
\hline -Dis of Veins, Lymphatics, Other & $451-459$ & 0 & 0 & 0 & 3 \\
\hline RESPIRATORY SYSTEM & $460-519$ & 3 & 0 & 0 & 45 \\
\hline -Acute Respiratory Infections & $460-466$ & 0 & 0 & 0 & 16 \\
\hline -Other Dis Upper Respiratory Tract & $470-478$ & 1 & 0 & 0 & 6 \\
\hline -Pneumonia \& Influenza & $480-488$ & 2 & 0 & 0 & 19 \\
\hline -Chronic Obstructive Dis & $490-496$ & 0 & 0 & 0 & 3 \\
\hline -Other Respiratory Dis & $510-519$ & 0 & 0 & 0 & 1 \\
\hline DIGESTIVE SYSTEM & $520-579$ & 1 & 0 & 0 & 25 \\
\hline -Oral Cavity, Saliva Glands, Jaw & $520-529$ & 0 & 0 & 0 & 3 \\
\hline -Esophagus, Stomach, Duodenum & $530-538$ & 0 & 0 & 0 & 2 \\
\hline -Appendicitis & $540-543$ & 0 & 0 & 0 & 4 \\
\hline -Hernias & $550-553$ & 0 & 0 & 0 & 2 \\
\hline
\end{tabular}

(Continued)

*Only those diagnostic categories and gender/job category combinations with at least one occurrence appear in this table. 


\section{Lawrence Livermore National Laboratory 2010}

Absence Data

Appendix I. Number of Diagnoses in Each Diagnostic Category by Gender and Job Category*

\begin{tabular}{|c|c|c|c|c|}
\hline & \multicolumn{3}{|c|}{ Women } \\
\hline & & \multicolumn{3}{|c|}{ Job Category } \\
\hline & & Professional & $\begin{array}{c}\text { Administrative } \\
\text { Support }\end{array}$ & Technical Support \\
\hline Diagnostic Category & ICD-9-CM Code & \multirow[b]{2}{*}{0} & \multirow[b]{2}{*}{1} & \multirow[b]{2}{*}{0} \\
\hline -Enteritis, Colitis & $555-558$ & & & \\
\hline -Other Intestinal Dis & $560-569$ & 0 & 4 & 1 \\
\hline -Other Digestive Dis & \begin{tabular}{|l|}
$570-579$ \\
\end{tabular} & 2 & 5 & 0 \\
\hline GENITOURINARY SYSTEM & $580-629$ & 1 & 16 & 2 \\
\hline -Other Urinary Dis & \begin{tabular}{|c|}
$590-599$ \\
\end{tabular} & 0 & 3 & 1 \\
\hline -Breast Disorders & $610-612$ & 0 & 1 & 0 \\
\hline -Other Female Disorders & $617-629$ & 1 & 12 & 1 \\
\hline PREGNANCY \& CHILDBIRTH & $630-679$ & 0 & 1 & $\overline{0}$ \\
\hline -Pregnancy with Abortive Outcome & $634-639$ & 0 & 1 & 0 \\
\hline SKIN AND SUBCUTANEOUS TISSUE & $680-709$ & 0 & 2 & $\overline{0}$ \\
\hline -Infections & $680-686$ & 0 & 1 & 0 \\
\hline -Other & \begin{tabular}{|l|}
$700-709$ \\
\end{tabular} & 0 & 1 & 0 \\
\hline $\begin{array}{l}\text { MUSCULOSKELETAL \& CONNECTIVE } \\
\text { TISSUE }\end{array}$ & \begin{tabular}{|l|}
$710-739$ \\
\end{tabular} & 11 & 30 & 2 \\
\hline -Arthropathies & $710-719$ & 4 & 13 & 0 \\
\hline -Dorsopathies & $720-724$ & 2 & 13 & 1 \\
\hline -Rheumatism, Excluding Back & 725-729 & 4 & 4 & 1 \\
\hline -Other Dis \& Acquired Deformities & $730-739$ & 1 & 0 & 0 \\
\hline $\begin{array}{l}\text { SYMPTOMS, SIGNS, \& ILL-DEFINED } \\
\text { CONDITIONS }\end{array}$ & 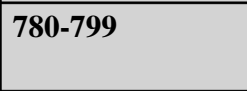 & 5 & 19 & 3 \\
\hline -Symptoms & $780-789$ & 5 & 19 & 3 \\
\hline INJURY \& POISONING & $800-999$ & 5 & 16 & 2 \\
\hline -Fracture - Upper Limb & \begin{tabular}{|l|}
$810-819$ \\
\end{tabular} & 0 & 3 & 0 \\
\hline -Fracture - Lower Limb & $820-829$ & 2 & 3 & 0 \\
\hline -Dislocation & 830-839 & 1 & 4 & 0 \\
\hline -Sprains \& Strains - Other & $840-845,848$ & 2 & 5 & 0 \\
\hline -Intracranial Injury & $850-854$ & 0 & 1 & $\overline{0}$ \\
\hline -Open Wound - Lower Limb & 890-897 & 0 & 0 & 0 \\
\hline -Contusion & $920-924$ & 0 & 0 & 1 \\
\hline -Complications \& Unspecified Injuries & 958-959 & 0 & 0 & 1 \\
\hline $\begin{array}{l}\text { HEALTH STATUS/HEALTH SERVICE } \\
\text { CONTACT }\end{array}$ & V01-V89 & 0 & 3 & 2 \\
\hline -Health Services Reproduction/Development & V20-V29 & 0 & 1 & 0 \\
\hline -Specific Procedures/Aftercare & V50-V59 & 0 & 2 & 2 \\
\hline
\end{tabular}

*Only those diagnostic categories and gender/job category combinations with at least one occurrence appear in this table. 


\section{Lawrence Livermore National Laboratory 2010}

Absence Data

Appendix I. Number of Diagnoses in Each Diagnostic Category by Gender and Job Category*

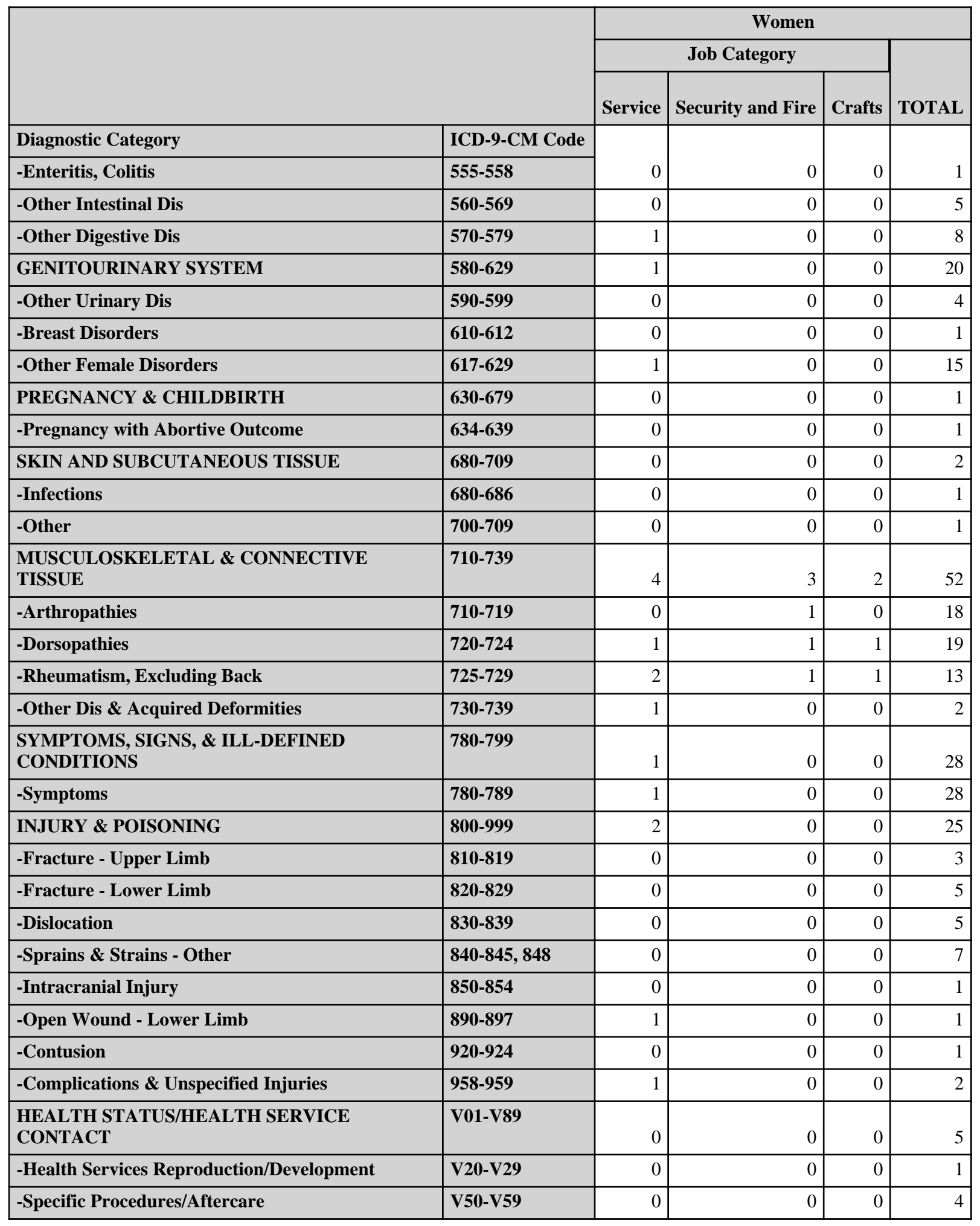

*Only those diagnostic categories and gender/job category combinations with at least one occurrence appear in this table. 
Lawrence Livermore National Laboratory 2010

Absence Data

Appendix I. Number of Diagnoses in Each Diagnostic Category by Gender and Job Category*

\begin{tabular}{|c|c|c|c|c|c|c|c|}
\hline & \multicolumn{7}{|c|}{ Women } \\
\hline & \multicolumn{6}{|c|}{ Job Category } & \multirow[b]{2}{*}{ TOTAI } \\
\hline & Professional & $\begin{array}{c}\text { Administrative } \\
\text { Support }\end{array}$ & Technical Support & Service & Security and Fire & Crafts & \\
\hline Diagnostic Category & \multirow[b]{2}{*}{45} & \multirow[b]{2}{*}{159} & \multirow[b]{2}{*}{20} & \multirow[b]{2}{*}{15} & \multirow[b]{2}{*}{3} & \multirow[b]{2}{*}{2} & \multirow[b]{2}{*}{244} \\
\hline Total & & & & & & & \\
\hline
\end{tabular}

*Only those diagnostic categories and gender/job category combinations with at least one occurrence appear in this table. 


\section{Lawrence Livermore National Laboratory 2010}

Absence Data

Appendix I. Number of Diagnoses in Each Diagnostic Category by Gender and Job Category*

\begin{tabular}{|c|c|c|c|c|}
\hline & \multicolumn{3}{|c|}{ Men } \\
\hline & & \multicolumn{3}{|c|}{ Job Category } \\
\hline & & Professional & $\begin{array}{c}\text { Administrative } \\
\text { Support }\end{array}$ & Technical Support \\
\hline Diagnostic Category & ICD-9-CM Code & \multirow[b]{2}{*}{5} & \multirow[b]{2}{*}{2} & \multirow[b]{2}{*}{0} \\
\hline INFECTIOUS \& PARASITIC DISEASES (DIS) & 001-139 & & & \\
\hline -Intestinal Infectious Dis & \begin{tabular}{|c|}
$001-009$ \\
\end{tabular} & 0 & 0 & $\overline{0}$ \\
\hline -Other Bacterial Dis & $030-041$ & 2 & 0 & 0 \\
\hline -Polio \& Other Viral CNS Dis & 045-049 & 1 & 0 & 0 \\
\hline -Viral Dis with Exanthem & $050-059$ & 0 & 1 & 0 \\
\hline -Other Viral Dis \& Chlamydiae & 070-079 & 2 & 1 & 0 \\
\hline MALIGNANT NEOPLASMS & $\begin{array}{l}140-208, \\
209.0-209.3 \\
230-234\end{array}$ & 14 & 3 & 3 \\
\hline -Digestive \& Peritoneal & $150-159$ & 4 & 1 & 2 \\
\hline -Respiratory \& Intrathoracic & $160-165$ & 0 & 0 & 1 \\
\hline -Genitourinary & 179-189 & 5 & 0 & 0 \\
\hline -Endocrine & $\begin{array}{l}193-194, \\
209.0-209.3\end{array}$ & 2 & 0 & 0 \\
\hline -Other \& Unspecified Sites & 195-199, 209.7 & 1 & 1 & 0 \\
\hline -Lymphatic \& Hematopoietic & $200-208$ & 2 & 1 & 0 \\
\hline BENIGN \& UNCERTAIN NEOPLASMS & $\begin{array}{l}209.4-209.6 \\
210-229,235-239\end{array}$ & 3 & 1 & 0 \\
\hline ENDOCRINE/METABOLIC/IMMUNITY & $240-279$ & 0 & 0 & 1 \\
\hline -Other Metabolic \& Immunity Disorders & $270-279$ & 0 & 0 & 1 \\
\hline BLOOD \& BLOOD-FORMING ORGANS & $280-289$ & 0 & 1 & 0 \\
\hline MENTAL DISORDERS & 290-319 & 1 & 3 & 0 \\
\hline -Non-Psychotic Disorders & $300-302,306-316$ & 0 & 2 & 0 \\
\hline -Alcohol Dependence & 303 & 0 & 1 & 0 \\
\hline -Drug Dependence & 304-305 & 1 & 0 & 0 \\
\hline NERVOUS SYSTEM (NS) \& SENSE ORGANS & 320-389 & 10 & 4 & 3 \\
\hline -Hereditary/Degenerative Central NS Dis & 330-337 & 1 & 0 & 0 \\
\hline -Disorders of Peripheral NS & $350-359$ & 2 & 0 & 2 \\
\hline -Disorders of Eye & $360-379$ & 5 & 3 & 1 \\
\hline -Diseases of Ear \& Mastoid & 380-389 & 2 & 1 & 0 \\
\hline CIRCULATORY SYSTEM & $390-459$ & 10 & 3 & 6 \\
\hline -Ischemic Heart Dis & $410-414$ & 3 & 2 & 1 \\
\hline -Other Heart Dis & $420-429$ & 4 & 0 & 1 \\
\hline -Cerebrovascular Dis & $430-438$ & 2 & 0 & 2 \\
\hline -Dis of Arteries \& Capillaries & $440-449$ & 0 & 1 & 1 \\
\hline -Dis of Veins, Lymphatics, Other & 451-459 & 1 & 0 & 1 \\
\hline RESPIRATORY SYSTEM & \begin{tabular}{|l|}
$460-519$ \\
\end{tabular} & 34 & 11 & 8 \\
\hline
\end{tabular}

(Continued)

*Only those diagnostic categories and gender/job category combinations with at least one occurrence appear in this table. 


\section{Lawrence Livermore National Laboratory 2010}

Absence Data

Appendix I. Number of Diagnoses in Each Diagnostic Category by Gender and Job Category*

\begin{tabular}{|c|c|c|c|c|c|}
\hline & & \multicolumn{4}{|c|}{ Men } \\
\hline & & \multicolumn{3}{|c|}{ Job Category } & \multirow[b]{2}{*}{ TOTAL } \\
\hline & & Service & Security and Fire & Crafts & \\
\hline Diagnostic Category & ICD-9-CM Code & & & & \\
\hline INFECTIOUS \& PARASITIC DISEASES (DIS) & 001-139 & 1 & 1 & 0 & 9 \\
\hline -Intestinal Infectious Dis & 001-009 & 1 & 0 & 0 & 1 \\
\hline -Other Bacterial Dis & 030-041 & 0 & 1 & 0 & 3 \\
\hline -Polio \& Other Viral CNS Dis & 045-049 & 0 & 0 & 0 & 1 \\
\hline -Viral Dis with Exanthem & 050-059 & 0 & 0 & 0 & 1 \\
\hline -Other Viral Dis \& Chlamydiae & 070-079 & 0 & 0 & 0 & 3 \\
\hline MALIGNANT NEOPLASMS & $\begin{array}{l}140-208 \\
209.0-209.3 \\
230-234\end{array}$ & 1 & 0 & 1 & 22 \\
\hline -Digestive \& Peritoneal & $150-159$ & 0 & 0 & 0 & 7 \\
\hline -Respiratory \& Intrathoracic & $160-165$ & 0 & 0 & 0 & 1 \\
\hline -Genitourinary & $179-189$ & 0 & 0 & 1 & 6 \\
\hline -Endocrine & $\begin{array}{l}193-194, \\
209.0-209.3\end{array}$ & 0 & 0 & 0 & 2 \\
\hline -Other \& Unspecified Sites & 195-199, 209.7 & 1 & 0 & 0 & 3 \\
\hline -Lymphatic \& Hematopoietic & $200-208$ & 0 & 0 & 0 & 3 \\
\hline BENIGN \& UNCERTAIN NEOPLASMS & $\begin{array}{l}209.4-209.6, \\
210-229,235-239\end{array}$ & 1 & 0 & 0 & 5 \\
\hline ENDOCRINE/METABOLIC/IMMUNITY & $240-279$ & 0 & 0 & 0 & 1 \\
\hline -Other Metabolic \& Immunity Disorders & 270-279 & 0 & 0 & 0 & 1 \\
\hline BLOOD \& BLOOD-FORMING ORGANS & $280-289$ & 0 & 0 & 0 & 1 \\
\hline MENTAL DISORDERS & $290-319$ & 0 & 1 & 0 & 5 \\
\hline -Non-Psychotic Disorders & $300-302,306-316$ & 0 & 1 & 0 & 3 \\
\hline -Alcohol Dependence & 303 & 0 & 0 & 0 & 1 \\
\hline -Drug Dependence & $304-305$ & 0 & 0 & 0 & 1 \\
\hline NERVOUS SYSTEM (NS) \& SENSE ORGANS & 320-389 & 2 & 2 & 0 & 21 \\
\hline -Hereditary/Degenerative Central NS Dis & 330-337 & 0 & 0 & 0 & 1 \\
\hline -Disorders of Peripheral NS & $350-359$ & 0 & 1 & 0 & 5 \\
\hline -Disorders of Eye & $360-379$ & 2 & 0 & 0 & 11 \\
\hline -Diseases of Ear \& Mastoid & 380-389 & 0 & 1 & 0 & 4 \\
\hline CIRCULATORY SYSTEM & $390-459$ & 1 & 0 & 1 & 21 \\
\hline -Ischemic Heart Dis & $410-414$ & 0 & 0 & 0 & 6 \\
\hline -Other Heart Dis & $420-429$ & 0 & 0 & 0 & 5 \\
\hline -Cerebrovascular Dis & $430-438$ & 0 & 0 & 0 & 4 \\
\hline -Dis of Arteries \& Capillaries & $440-449$ & 1 & 0 & 0 & 3 \\
\hline -Dis of Veins, Lymphatics, Other & $451-459$ & 0 & 0 & 1 & 3 \\
\hline RESPIRATORY SYSTEM & $460-519$ & 4 & 4 & 3 & 64 \\
\hline
\end{tabular}

(Continued)

*Only those diagnostic categories and gender/job category combinations with at least one occurrence appear in this table. 


\section{Lawrence Livermore National Laboratory 2010}

Absence Data

Appendix I. Number of Diagnoses in Each Diagnostic Category by Gender and Job Category*

\begin{tabular}{|c|c|c|c|c|}
\hline & \multicolumn{3}{|c|}{ Men } \\
\hline & & \multicolumn{3}{|c|}{ Job Category } \\
\hline & & Professional & $\begin{array}{l}\text { Administrative } \\
\text { Support }\end{array}$ & Technical Support \\
\hline Diagnostic Category & ICD-9-CM Code & \multirow[b]{2}{*}{16} & \multirow[b]{2}{*}{5} & \multirow[b]{2}{*}{1} \\
\hline -Acute Respiratory Infections & $460-466$ & & & \\
\hline -Other Dis Upper Respiratory Tract & $470-478$ & 4 & 2 & 1 \\
\hline -Pneumonia \& Influenza & $480-488$ & 13 & 3 & 6 \\
\hline -Chronic Obstructive Dis & $490-496$ & 1 & 1 & 0 \\
\hline DIGESTIVE SYSTEM & $520-579$ & 21 & 7 & 5 \\
\hline -Oral Cavity, Saliva Glands, Jaw & $520-529$ & 1 & 0 & 2 \\
\hline -Esophagus, Stomach, Duodenum & $530-538$ & 3 & 1 & 0 \\
\hline -Appendicitis & $540-543$ & 2 & 0 & 2 \\
\hline -Hernias & $550-553$ & 7 & 3 & 1 \\
\hline -Other Intestinal Dis & $560-569$ & 3 & 0 & 0 \\
\hline -Other Digestive Dis & $570-579$ & 5 & 3 & 0 \\
\hline GENITOURINARY SYSTEM & $580-629$ & 6 & 1 & 1 \\
\hline -Other Urinary Dis & \begin{tabular}{|l|}
$590-599$ \\
\end{tabular} & 3 & 0 & 1 \\
\hline -Male Genital Organ Dis & $600-608$ & 3 & 1 & 0 \\
\hline SKIN AND SUBCUTANEOUS TISSUE & 680-709 & 1 & 1 & 0 \\
\hline -Infections & $680-686$ & 0 & 0 & 0 \\
\hline -Other & \begin{tabular}{|c|}
$700-709$ \\
\end{tabular} & 1 & 1 & 0 \\
\hline $\begin{array}{l}\text { MUSCULOSKELETAL \& CONNECTIVE } \\
\text { TISSUE }\end{array}$ & $710-739$ & 31 & 16 & 7 \\
\hline -Arthropathies & $710-719$ & 18 & 3 & 4 \\
\hline -Dorsopathies & $720-724$ & 8 & 8 & 2 \\
\hline -Rheumatism, Excluding Back & 725-729 & 5 & 5 & 1 \\
\hline -Other Dis \& Acquired Deformities & $730-739$ & 0 & 0 & 0 \\
\hline $\begin{array}{l}\text { SYMPTOMS, SIGNS, \& ILL-DEFINED } \\
\text { CONDITIONS }\end{array}$ & 780-799 & 13 & 5 & 7 \\
\hline -Symptoms & 780-789 & 13 & 5 & 7 \\
\hline INJURY \& POISONING & $800-999$ & 24 & 7 & 14 \\
\hline -Fracture - Upper Limb & $810-819$ & 1 & 2 & 0 \\
\hline -Fracture - Lower Limb & 820-829 & 3 & 0 & 2 \\
\hline -Dislocation & $830-839$ & 4 & 0 & 0 \\
\hline -Sprains \& Strains - Back & 846-847 & 0 & 0 & 3 \\
\hline -Sprains \& Strains - Other & $840-845,848$ & 12 & 4 & 7 \\
\hline -Open Wound - Head, Neck, Trunk & $870-879$ & 1 & 0 & 0 \\
\hline -Open Wound - Lower Limb & $890-897$ & 0 & 0 & $\overline{0}$ \\
\hline -Superficial Injury & 910-919 & 0 & 0 & 1 \\
\hline -Contusion & $920-924$ & 2 & 0 & 1 \\
\hline -Complications \& Unspecified Injuries & 958-959 & 1 & 1 & 0 \\
\hline
\end{tabular}

(Continued)

*Only those diagnostic categories and gender/job category combinations with at least one occurrence appear in this table. 


\section{Lawrence Livermore National Laboratory 2010}

Absence Data

Appendix I. Number of Diagnoses in Each Diagnostic Category by Gender and Job Category*

\begin{tabular}{|c|c|c|c|c|c|}
\hline & & \multicolumn{4}{|c|}{ Men } \\
\hline & & \multicolumn{3}{|c|}{ Job Category } & \multirow[b]{2}{*}{ TOTAL } \\
\hline & & Service & Security and Fire & Crafts & \\
\hline Diagnostic Category & ICD-9-CM Code & \multirow[b]{2}{*}{0} & \multirow[b]{2}{*}{1} & \multirow[b]{2}{*}{2} & \multirow[b]{2}{*}{25} \\
\hline -Acute Respiratory Infections & $460-466$ & & & & \\
\hline -Other Dis Upper Respiratory Tract & $470-478$ & 1 & 0 & 0 & 8 \\
\hline -Pneumonia \& Influenza & $480-488$ & 2 & 3 & 0 & 27 \\
\hline -Chronic Obstructive Dis & $490-496$ & 1 & 0 & 1 & 4 \\
\hline DIGESTIVE SYSTEM & $520-579$ & 5 & 1 & 2 & 41 \\
\hline -Oral Cavity, Saliva Glands, Jaw & $520-529$ & 0 & 0 & 0 & 3 \\
\hline -Esophagus, Stomach, Duodenum & $530-538$ & 0 & 0 & 0 & 4 \\
\hline -Appendicitis & $540-543$ & 0 & 0 & 0 & 4 \\
\hline -Hernias & $550-553$ & 4 & 0 & 2 & 17 \\
\hline -Other Intestinal Dis & $560-569$ & 0 & 1 & 0 & 4 \\
\hline -Other Digestive Dis & $570-579$ & 1 & 0 & 0 & 9 \\
\hline GENITOURINARY SYSTEM & $580-629$ & 2 & 0 & 0 & 10 \\
\hline -Other Urinary Dis & $590-599$ & 2 & 0 & 0 & 6 \\
\hline -Male Genital Organ Dis & $600-608$ & 0 & 0 & 0 & 4 \\
\hline SKIN AND SUBCUTANEOUS TISSUE & 680-709 & 0 & 0 & 1 & 3 \\
\hline -Infections & $680-686$ & 0 & 0 & 1 & 1 \\
\hline -Other & 700-709 & 0 & 0 & 0 & 2 \\
\hline $\begin{array}{l}\text { MUSCULOSKELETAL \& CONNECTIVE } \\
\text { TISSUE }\end{array}$ & 710-739 & 4 & 4 & 7 & 69 \\
\hline -Arthropathies & $710-719$ & 2 & 4 & 3 & 34 \\
\hline -Dorsopathies & $720-724$ & 1 & 0 & 4 & 23 \\
\hline -Rheumatism, Excluding Back & 725-729 & 0 & 0 & 0 & 11 \\
\hline -Other Dis \& Acquired Deformities & $730-739$ & 1 & 0 & 0 & 1 \\
\hline $\begin{array}{l}\text { SYMPTOMS, SIGNS, \& ILL-DEFINED } \\
\text { CONDITIONS }\end{array}$ & 780-799 & 0 & 0 & 2 & 27 \\
\hline -Symptoms & 780-789 & 0 & 0 & 2 & 27 \\
\hline INJURY \& POISONING & $800-999$ & 5 & 4 & 5 & 59 \\
\hline -Fracture - Upper Limb & $810-819$ & 1 & 1 & 0 & 5 \\
\hline -Fracture - Lower Limb & 820-829 & 1 & 0 & 0 & 6 \\
\hline -Dislocation & 830-839 & 1 & 0 & 0 & 5 \\
\hline -Sprains \& Strains - Back & 846-847 & 2 & 0 & 0 & 5 \\
\hline -Sprains \& Strains - Other & $840-845,848$ & 0 & 2 & 2 & 27 \\
\hline -Open Wound - Head, Neck, Trunk & 870-879 & 0 & 0 & 0 & 1 \\
\hline -Open Wound - Lower Limb & 890-897 & 0 & 1 & 0 & 1 \\
\hline -Superficial Injury & 910-919 & 0 & 0 & 1 & 2 \\
\hline -Contusion & $920-924$ & 0 & 0 & 1 & 4 \\
\hline -Complications \& Unspecified Injuries & 958-959 & 0 & 0 & 1 & 3 \\
\hline
\end{tabular}

(Continued)

*Only those diagnostic categories and gender/job category combinations with at least one occurrence appear in this table. 
Lawrence Livermore National Laboratory 2010

Absence Data

Appendix I. Number of Diagnoses in Each Diagnostic Category by Gender and Job Category*

\begin{tabular}{|c|c|c|c|c|}
\hline & \multicolumn{3}{|c|}{ Men } \\
\hline & & \multicolumn{3}{|c|}{ Job Category } \\
\hline & & Professional & \begin{tabular}{|c|} 
Administrative \\
Support
\end{tabular} & Technical Support \\
\hline Diagnostic Category & ICD-9-CM Code & \multirow[b]{2}{*}{4} & \multirow[b]{2}{*}{0} & \multirow[b]{2}{*}{1} \\
\hline $\begin{array}{l}\text { HEALTH STATUS/HEALTH SERVICE } \\
\text { CONTACT }\end{array}$ & V01-V89 & & & \\
\hline -Personal \& Family History & V10-V19 & 1 & 0 & 0 \\
\hline -Health Services Reproduction/Development & V20-V29 & 1 & 0 & 1 \\
\hline -Examination \& Investigation & V70-V82 & 2 & 0 & 0 \\
\hline
\end{tabular}

*Only those diagnostic categories and gender/job category combinations with at least one occurrence appear in this table. 
Lawrence Livermore National Laboratory 2010

Absence Data

Appendix I. Number of Diagnoses in Each Diagnostic Category by Gender and Job Category*

\begin{tabular}{|c|c|c|c|c|c|}
\hline & & \multicolumn{4}{|c|}{ Men } \\
\hline & & \multicolumn{3}{|c|}{ Job Category } & \multirow[b]{2}{*}{ TOTAL } \\
\hline & & Service & Security and Fire & Crafts & \\
\hline Diagnostic Category & ICD-9-CM Code & & & & \\
\hline $\begin{array}{l}\text { HEALTH STATUS/HEALTH SERVICE } \\
\text { CONTACT }\end{array}$ & V01-V89 & 0 & 0 & 1 & 6 \\
\hline -Personal \& Family History & V10-V19 & 0 & 0 & 0 & 1 \\
\hline -Health Services Reproduction/Development & V20-V29 & 0 & 0 & 0 & 2 \\
\hline -Examination \& Investigation & V70-V82 & 0 & 0 & 1 & 3 \\
\hline
\end{tabular}

\begin{tabular}{|c|c|c|c|c|c|c|c|}
\hline & \multicolumn{7}{|c|}{ Men } \\
\hline & \multicolumn{6}{|c|}{ Job Category } & \multirow[b]{2}{*}{ TOTAL } \\
\hline & Professional & $\begin{array}{c}\text { Administrative } \\
\text { Support }\end{array}$ & Technical Support & Service & Security and Fire & Crafts & \\
\hline Diagnostic Category & \multirow[b]{2}{*}{177} & \multirow[b]{2}{*}{65} & \multirow[b]{2}{*}{56} & \multirow[b]{2}{*}{26} & \multirow[b]{2}{*}{17} & \multirow[b]{2}{*}{23} & \multirow[b]{2}{*}{364} \\
\hline Total & & & & & & & \\
\hline
\end{tabular}

*Only those diagnostic categories and gender/job category combinations with at least one occurrence appear in this table. 
Lawrence Livermore National Laboratory 2010

Absence Data

Appendix J. Total Number of Calendar Days Absent in Each Diagnostic Category by Gender and Job Category*

\begin{tabular}{|c|c|c|c|c|}
\hline & & \multicolumn{3}{|c|}{ Women } \\
\hline & & \multicolumn{3}{|c|}{ Job Category } \\
\hline & & Professional & $\begin{array}{l}\text { Administrative } \\
\text { Support }\end{array}$ & Technical Support \\
\hline Diagnostic Category & ICD-9-CM Code & \multirow[b]{2}{*}{10} & \multirow[b]{2}{*}{70} & \multirow[b]{2}{*}{11} \\
\hline INFECTIOUS \& PARASITIC DISEASES (DIS) & 001-139 & & & \\
\hline MALIGNANT NEOPLASMS & $\begin{array}{l}140-208, \\
209.0-209.3 \\
230-234\end{array}$ & 31 & 22 & 0 \\
\hline BENIGN \& UNCERTAIN NEOPLASMS & $\begin{array}{l}209.4-209.6 \\
210-229,235-239\end{array}$ & 33 & 137 & 9 \\
\hline ENDOCRINE/METABOLIC/IMMUNITY & $240-279$ & 0 & 77 & 0 \\
\hline MENTAL DISORDERS & $290-319$ & 0 & 24 & 0 \\
\hline NERVOUS SYSTEM (NS) \& SENSE ORGANS & 320-389 & 152 & 120 & 9 \\
\hline CIRCULATORY SYSTEM & $390-459$ & 19 & 213 & 0 \\
\hline RESPIRATORY SYSTEM & $460-519$ & 88 & 371 & 457 \\
\hline DIGESTIVE SYSTEM & $520-579$ & 60 & 386 & 27 \\
\hline GENITOURINARY SYSTEM & $580-629$ & 50 & 603 & 15 \\
\hline PREGNANCY \& CHILDBIRTH & $630-679$ & 0 & 12 & 0 \\
\hline SKIN AND SUBCUTANEOUS TISSUE & 680-709 & 0 & 10 & 0 \\
\hline $\begin{array}{l}\text { MUSCULOSKELETAL \& CONNECTIVE } \\
\text { TISSUE }\end{array}$ & $710-739$ & 217 & 1,792 & 64 \\
\hline $\begin{array}{l}\text { SYMPTOMS, SIGNS, \& ILL-DEFINED } \\
\text { CONDITIONS }\end{array}$ & $780-799$ & 80 & 527 & 473 \\
\hline INJURY \& POISONING & $800-999$ & 125 & 613 & 26 \\
\hline
\end{tabular}

\footnotetext{
*Absences with >1 ICD-9-CM code in the same diagnostic category were counted only once. Only those diagnostic categories and gender/job category combinations with at least one occurrence appear in this table.
} 
Lawrence Livermore National Laboratory 2010

Absence Data

Appendix J. Total Number of Calendar Days Absent in Each Diagnostic Category by Gender and Job Category*

\begin{tabular}{|c|c|c|c|c|c|}
\hline & & \multicolumn{4}{|c|}{ Women } \\
\hline & & \multicolumn{3}{|c|}{ Job Category } & \multirow[b]{2}{*}{ TOTAL } \\
\hline & & Service & Security and Fire & Crafts & \\
\hline Diagnostic Category & ICD-9-CM Code & \multirow[b]{2}{*}{0} & \multirow[b]{2}{*}{0} & \multirow[b]{2}{*}{0} & \multirow[b]{2}{*}{91} \\
\hline INFECTIOUS \& PARASITIC DISEASES (DIS) & 001-139 & & & & \\
\hline MALIGNANT NEOPLASMS & $\begin{array}{l}140-208 \\
209.0-209.3 \\
230-234\end{array}$ & 43 & 0 & 0 & 96 \\
\hline BENIGN \& UNCERTAIN NEOPLASMS & $\begin{array}{l}209.4-209.6 \\
210-229,235-239\end{array}$ & 0 & 0 & 0 & 179 \\
\hline ENDOCRINE/METABOLIC/IMMUNITY & $240-279$ & 0 & 0 & 0 & 77 \\
\hline MENTAL DISORDERS & $290-319$ & 0 & 0 & 0 & 24 \\
\hline NERVOUS SYSTEM (NS) \& SENSE ORGANS & $320-389$ & 10 & 0 & 0 & 291 \\
\hline CIRCULATORY SYSTEM & $390-459$ & 13 & 0 & 0 & 245 \\
\hline RESPIRATORY SYSTEM & $460-519$ & 26 & 0 & 0 & 942 \\
\hline DIGESTIVE SYSTEM & $520-579$ & 18 & 0 & 0 & 491 \\
\hline GENITOURINARY SYSTEM & $580-629$ & 51 & 0 & 0 & 719 \\
\hline PREGNANCY \& CHILDBIRTH & $630-679$ & 0 & 0 & 0 & 12 \\
\hline SKIN AND SUBCUTANEOUS TISSUE & $680-709$ & 0 & 0 & 0 & 10 \\
\hline $\begin{array}{l}\text { MUSCULOSKELETAL \& CONNECTIVE } \\
\text { TISSUE }\end{array}$ & $710-739$ & 288 & 161 & 6 & 2,528 \\
\hline $\begin{array}{l}\text { SYMPTOMS, SIGNS, \& ILL-DEFINED } \\
\text { CONDITIONS }\end{array}$ & $780-799$ & 6 & 0 & 0 & 1,086 \\
\hline INJURY \& POISONING & 800-999 & 5 & 0 & 0 & 769 \\
\hline
\end{tabular}

\footnotetext{
*Absences with >1 ICD-9-CM code in the same diagnostic category were counted only once. Only those diagnostic categories and gender/job category combinations with at least one occurrence appear in this table.
} 
Lawrence Livermore National Laboratory 2010

Absence Data

Appendix J. Total Number of Calendar Days Absent in Each Diagnostic Category by Gender and Job Category*

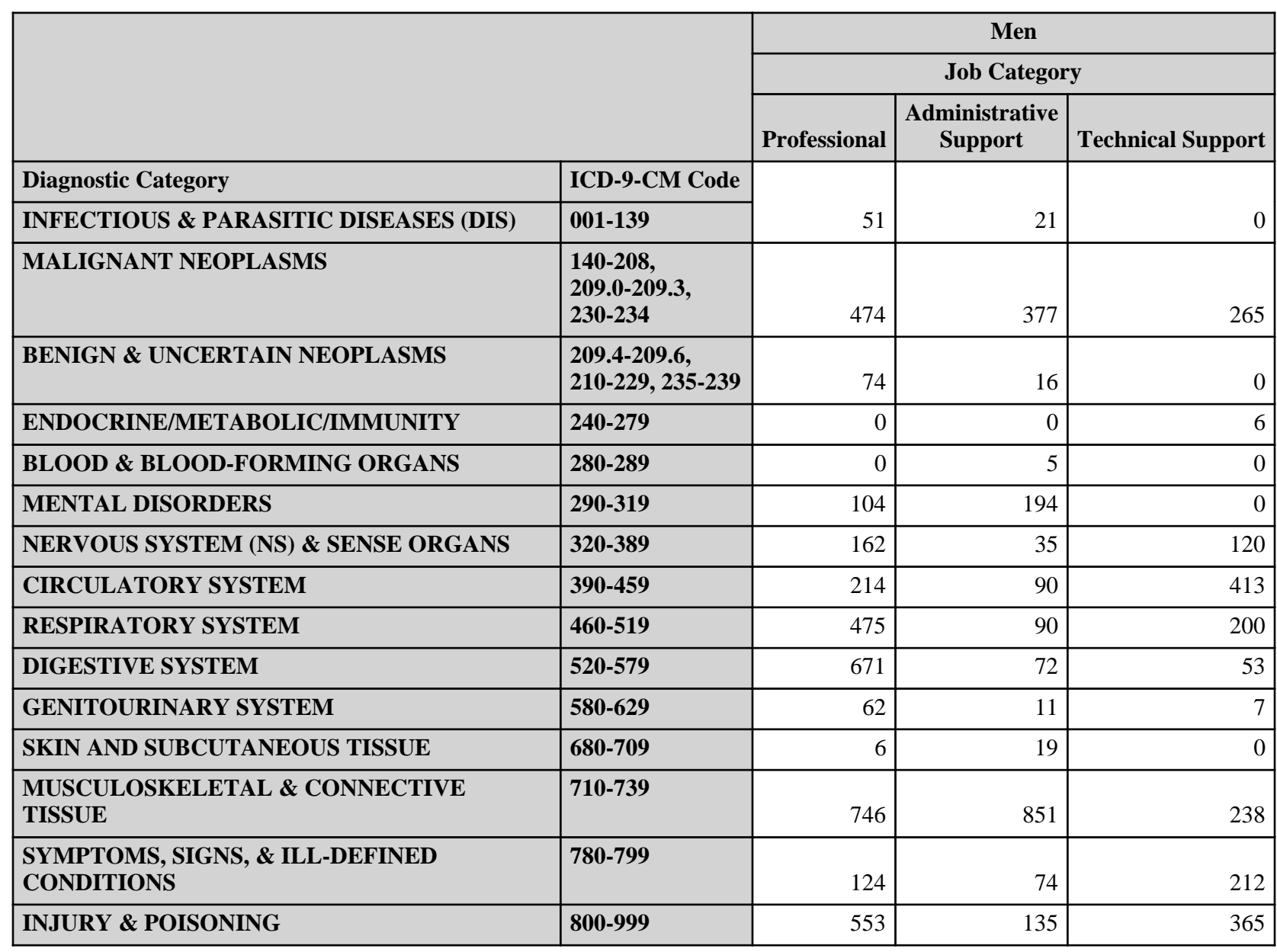

\footnotetext{
*Absences with >1 ICD-9-CM code in the same diagnostic category were counted only once. Only those diagnostic categories and gender/job category combinations with at least one occurrence appear in this table.
} 
Lawrence Livermore National Laboratory 2010

Absence Data

Appendix J. Total Number of Calendar Days Absent in Each Diagnostic Category by Gender and Job Category*

\begin{tabular}{|c|c|c|c|c|c|}
\hline & & \multicolumn{4}{|c|}{ Men } \\
\hline & & \multicolumn{3}{|c|}{ Job Category } & \multirow[b]{2}{*}{ TOTAI } \\
\hline & & Service & Security and Fire & Crafts & \\
\hline Diagnostic Category & ICD-9-CM Code & \multirow[b]{2}{*}{11} & \multirow[b]{2}{*}{13} & \multirow[b]{2}{*}{0} & \multirow[b]{2}{*}{96} \\
\hline INFECTIOUS \& PARASITIC DISEASES (DIS) & 001-139 & & & & \\
\hline MALIGNANT NEOPLASMS & $\begin{array}{l}140-208, \\
209.0-209.3 \\
230-234\end{array}$ & 7 & 0 & 82 & 1,205 \\
\hline BENIGN \& UNCERTAIN NEOPLASMS & $\begin{array}{l}209.4-209.6 \\
210-229,235-239\end{array}$ & 13 & 0 & 0 & 103 \\
\hline ENDOCRINE/METABOLIC/IMMUNITY & $240-279$ & 0 & 0 & 0 & 6 \\
\hline BLOOD \& BLOOD-FORMING ORGANS & $280-289$ & 0 & 0 & 0 & 5 \\
\hline MENTAL DISORDERS & $290-319$ & 0 & 17 & 0 & 315 \\
\hline NERVOUS SYSTEM (NS) \& SENSE ORGANS & 320-389 & 23 & 24 & 0 & 364 \\
\hline CIRCULATORY SYSTEM & $390-459$ & 5 & 0 & 7 & 729 \\
\hline RESPIRATORY SYSTEM & $460-519$ & 37 & 31 & 27 & 860 \\
\hline DIGESTIVE SYSTEM & $520-579$ & 114 & 8 & 59 & 977 \\
\hline GENITOURINARY SYSTEM & $580-629$ & 15 & 0 & 0 & 95 \\
\hline SKIN AND SUBCUTANEOUS TISSUE & $680-709$ & 0 & 0 & 17 & 42 \\
\hline $\begin{array}{l}\text { MUSCULOSKELETAL \& CONNECTIVE } \\
\text { TISSUE }\end{array}$ & $710-739$ & 62 & 395 & 1,074 & 3,366 \\
\hline $\begin{array}{l}\text { SYMPTOMS, SIGNS, \& ILL-DEFINED } \\
\text { CONDITIONS }\end{array}$ & $780-799$ & 0 & 0 & 13 & 423 \\
\hline INJURY \& POISONING & 800-999 & 485 & 166 & 120 & 1,824 \\
\hline
\end{tabular}

\footnotetext{
*Absences with >1 ICD-9-CM code in the same diagnostic category were counted only once. Only those diagnostic categories and gender/job category combinations with at least one occurrence appear in this table.
} 


\section{Lawrence Livermore National Laboratory 2010}

Absence Data

Appendix K. Age-Adjusted Illness and Injury Rates by Diagnostic Category*

Part 1. Men

\begin{tabular}{|c|c|c|c|c|c|}
\hline & & $\begin{array}{l}\text { Number of } \\
\text { Diagnoses }\end{array}$ & $\begin{array}{c}\text { Age-Adjusted } \\
\text { Rate per } 1,000 * *\end{array}$ & $\begin{array}{c}\text { Lower } 95 \% \\
\text { Confidence } \\
\text { Limit per 1,000 }\end{array}$ & $\begin{array}{c}\text { Upper } 95 \% \\
\text { Confidence } \\
\text { Limit per 1,000 }\end{array}$ \\
\hline Diagnostic Category & ICD-9-CM Code & \multirow[b]{2}{*}{9} & \multirow[b]{2}{*}{3.0} & \multirow[b]{2}{*}{0.9} & \multirow[b]{2}{*}{9.5} \\
\hline INFECTIOUS \& PARASITIC DISEASES (DIS) & 001-139 & & & & \\
\hline -Intestinal Infectious Dis & 001-009 & 1 & 0.2 & 0.0 & 1.3 \\
\hline -Other Bacterial Dis & 030-041 & 3 & 2.1 & 0.4 & 10.6 \\
\hline -Polio \& Other Viral CNS Dis & $045-049$ & 1 & 0.2 & 0.0 & 1.4 \\
\hline -Viral Dis with Exanthem & 050-059 & 1 & 0.1 & 0.0 & 0.7 \\
\hline -Other Viral Dis \& Chlamydiae & 070-079 & 3 & 0.5 & 0.1 & 1.5 \\
\hline MALIGNANT NEOPLASMS & $\begin{array}{l}140-208, \\
209.0-209.3 \\
230-234\end{array}$ & 22 & 2.4 & 1.5 & 3.6 \\
\hline -Digestive \& Peritoneal & $150-159$ & 7 & 0.8 & 0.4 & $\overline{1.6}$ \\
\hline -Respiratory \& Intrathoracic & $160-165$ & 1 & 0.1 & 0.0 & 0.7 \\
\hline -Genitourinary & 179-189 & 6 & 0.7 & 0.3 & 1.5 \\
\hline -Endocrine & $\begin{array}{l}193-194, \\
209.0-209.3\end{array}$ & 2 & 0.2 & 0.0 & 0.8 \\
\hline -Other \& Unspecified Sites & 195-199, 209.7 & 3 & 0.4 & 0.1 & 1.2 \\
\hline -Lymphatic \& Hematopoietic & $200-208$ & 3 & 0.3 & 0.1 & 0.9 \\
\hline BENIGN \& UNCERTAIN NEOPLASMS & $\begin{array}{l}209.4-209.6 \\
210-229,235-239\end{array}$ & 5 & 0.6 & 0.3 & 1.6 \\
\hline ENDOCRINE/METABOLIC/IMMUNITY & $240-279$ & 1 & 0.2 & 0.0 & 1.4 \\
\hline -Other Metabolic \& Immunity Disorders & $270-279$ & 1 & 0.2 & 0.0 & 1.4 \\
\hline BLOOD \& BLOOD-FORMING ORGANS & $280-289$ & 1 & 0.1 & 0.0 & 0.7 \\
\hline MENTAL DISORDERS & $290-319$ & 5 & 1.4 & 0.5 & 3.6 \\
\hline -Non-Psychotic Disorders & $300-302,306-316$ & 3 & 0.8 & 0.2 & 2.9 \\
\hline -Alcohol Dependence & 303 & 1 & 0.4 & 0.1 & 2.7 \\
\hline -Drug Dependence & 304-305 & 1 & 0.2 & 0.0 & 1.4 \\
\hline NERVOUS SYSTEM (NS) \& SENSE ORGANS & 320-389 & 21 & 3.4 & 2.1 & 5.4 \\
\hline -Hereditary/Degenerative Central NS Dis & 330-337 & 1 & 0.1 & 0.0 & 0.7 \\
\hline -Disorders of Peripheral NS & $350-359$ & 5 & 0.7 & 0.3 & 1.7 \\
\hline -Disorders of Eye & $360-379$ & 11 & 1.7 & 0.9 & 3.1 \\
\hline -Diseases of Ear \& Mastoid & 380-389 & 4 & 1.0 & 0.3 & 2.7 \\
\hline CIRCULATORY SYSTEM & $390-459$ & 21 & 3.0 & 1.9 & 4.8 \\
\hline -Ischemic Heart Dis & $410-414$ & 6 & 0.8 & 0.4 & 1.9 \\
\hline -Other Heart Dis & $420-429$ & 5 & 0.6 & 0.3 & 1.6 \\
\hline -Cerebrovascular Dis & $430-438$ & 4 & 0.6 & 0.2 & 1.6 \\
\hline -Dis of Arteries \& Capillaries & $440-449$ & 3 & 0.4 & 0.1 & 1.3 \\
\hline -Dis of Veins, Lymphatics, Other & $451-459$ & 3 & 0.6 & 0.1 & 2.2 \\
\hline RESPIRATORY SYSTEM & $460-519$ & 64 & 15.2 & 10.0 & 23.2 \\
\hline
\end{tabular}

(Continued)

* Only those diagnostic categories with at least one occurrence appear in this table.

**Standardized to age distribution of 2000 U.S. population. 


\section{Lawrence Livermore National Laboratory 2010}

Absence Data

Appendix K. Age-Adjusted Illness and Injury Rates by Diagnostic Category*

Part 1. Men

\begin{tabular}{|c|c|c|c|c|c|}
\hline & & $\begin{array}{l}\text { Number of } \\
\text { Diagnoses }\end{array}$ & $\begin{array}{c}\text { Age-Adjusted } \\
\text { Rate per } 1,000 * *\end{array}$ & $\begin{array}{c}\text { Lower } 95 \% \\
\text { Confidence } \\
\text { Limit per 1,000 }\end{array}$ & $\begin{array}{c}\text { Upper } 95 \% \\
\text { Confidence } \\
\text { Limit per 1,000 }\end{array}$ \\
\hline Diagnostic Category & ICD-9-CM Code & \multirow[b]{2}{*}{25} & \multirow[b]{2}{*}{4.4} & \multirow[b]{2}{*}{2.9} & \multirow[b]{2}{*}{6.8} \\
\hline -Acute Respiratory Infections & $460-466$ & & & & \\
\hline -Other Dis Upper Respiratory Tract & $470-478$ & 8 & 1.2 & 0.6 & 2.8 \\
\hline -Pneumonia \& Influenza & $480-488$ & 27 & 9.0 & 4.6 & 17.5 \\
\hline -Chronic Obstructive Dis & $490-496$ & 4 & 0.6 & 0.2 & 1.6 \\
\hline DIGESTIVE SYSTEM & $520-579$ & 41 & 6.6 & 4.6 & 9.2 \\
\hline -Oral Cavity, Saliva Glands, Jaw & $520-529$ & 3 & 0.7 & 0.2 & 2.4 \\
\hline -Esophagus, Stomach, Duodenum & $530-538$ & 4 & 0.6 & 0.2 & 1.6 \\
\hline -Appendicitis & $540-543$ & 4 & 0.4 & 0.1 & 1.0 \\
\hline -Hernias & $550-553$ & 17 & 2.9 & 1.7 & 5.0 \\
\hline -Other Intestinal Dis & $560-569$ & 4 & 0.7 & 0.2 & 2.2 \\
\hline -Other Digestive Dis & $570-579$ & 9 & 1.3 & 0.7 & 2.6 \\
\hline GENITOURINARY SYSTEM & $580-629$ & 10 & 1.7 & 0.9 & 3.2 \\
\hline -Other Urinary Dis & $590-599$ & 6 & 1.1 & 0.5 & 2.4 \\
\hline -Male Genital Organ Dis & $600-608$ & 4 & 0.6 & 0.2 & 1.7 \\
\hline SKIN AND SUBCUTANEOUS TISSUE & $680-709$ & 3 & 0.5 & 0.1 & 1.5 \\
\hline -Infections & $680-686$ & 1 & 0.2 & 0.0 & 1.4 \\
\hline -Other & 700-709 & 2 & 0.3 & 0.1 & 1.2 \\
\hline $\begin{array}{l}\text { MUSCULOSKELETAL \& CONNECTIVE } \\
\text { TISSUE }\end{array}$ & 710-739 & 69 & 11.4 & 7.9 & 16.5 \\
\hline -Arthropathies & 710-719 & 34 & 6.3 & 3.5 & 11.4 \\
\hline -Dorsopathies & $720-724$ & 23 & 3.4 & 2.2 & 5.3 \\
\hline -Rheumatism, Excluding Back & 725-729 & 11 & 1.6 & 0.8 & 3.1 \\
\hline -Other Dis \& Acquired Deformities & $730-739$ & 1 & 0.1 & 0.0 & 0.7 \\
\hline $\begin{array}{l}\text { SYMPTOMS, SIGNS, \& ILL-DEFINED } \\
\text { CONDITIONS }\end{array}$ & 780-799 & 27 & 4.3 & 2.8 & 6.5 \\
\hline -Symptoms & 780-789 & 27 & 4.3 & 2.8 & 6.5 \\
\hline INJURY \& POISONING & 800-999 & 59 & 14.7 & 9.5 & 22.7 \\
\hline -Fracture - Upper Limb & $810-819$ & 5 & 1.0 & 0.4 & 2.6 \\
\hline -Fracture - Lower Limb & 820-829 & 6 & 1.0 & 0.4 & 2.3 \\
\hline -Dislocation & $830-839$ & 5 & 1.1 & 0.4 & 2.8 \\
\hline -Sprains \& Strains - Back & 846-847 & 5 & 0.8 & 0.3 & 1.9 \\
\hline -Sprains \& Strains - Other & $840-845,848$ & 27 & 9.6 & 5.1 & 18.2 \\
\hline -Open Wound - Head, Neck, Trunk & 870-879 & 2 & 0.2 & 0.0 & 0.8 \\
\hline -Superficial Injury & 910-919 & 2 & 0.2 & 0.0 & 0.8 \\
\hline -Contusion & $920-924$ & 4 & 0.4 & 0.1 & 1.0 \\
\hline
\end{tabular}

(Continued)

* Only those diagnostic categories with at least one occurrence appear in this table.

**Standardized to age distribution of 2000 U.S. population. 
Lawrence Livermore National Laboratory 2010

Absence Data

Appendix K. Age-Adjusted IIIness and Injury Rates by Diagnostic Category*

Part 1. Men

\begin{tabular}{|c|c|c|c|c|c|}
\hline & & $\begin{array}{l}\text { Number of } \\
\text { Diagnoses }\end{array}$ & $\begin{array}{c}\text { Age-Adjusted } \\
\text { Rate per } 1,000^{* * *}\end{array}$ & $\begin{array}{c}\text { Lower } 95 \% \\
\text { Confidence } \\
\text { Limit per } 1,000\end{array}$ & $\begin{array}{c}\text { Upper 95\% } \\
\text { Confidence } \\
\text { Limit per 1,000 }\end{array}$ \\
\hline Diagnostic Category & ICD-9-CM Code & \multirow[b]{2}{*}{3} & \multirow[b]{2}{*}{0.5} & \multirow[b]{2}{*}{0.1} & \multirow[b]{2}{*}{1.5} \\
\hline -Complications \& Unspecified Injuries & 958-959 & & & & \\
\hline Total & & 358 & 68.4 & 57.9 & 80.7 \\
\hline
\end{tabular}

* Only those diagnostic categories with at least one occurrence appear in this table.

**Standardized to age distribution of 2000 U.S. population. 


\section{Lawrence Livermore National Laboratory 2010}

Absence Data

Appendix K. Age-Adjusted Illness and Injury Rates by Diagnostic Category*

Part 2. Women

\begin{tabular}{|c|c|c|c|c|c|}
\hline & & $\begin{array}{l}\text { Number of } \\
\text { Diagnoses }\end{array}$ & $\begin{array}{c}\text { Age-Adjusted } \\
\text { Rate per } 1,000 * *\end{array}$ & $\begin{array}{c}\text { Lower } 95 \% \\
\text { Confidence } \\
\text { Limit per 1,000 }\end{array}$ & $\begin{array}{c}\text { Upper } 95 \% \\
\text { Confidence } \\
\text { Limit per 1,000 }\end{array}$ \\
\hline Diagnostic Category & ICD-9-CM Code & & & & \\
\hline INFECTIOUS \& PARASITIC DISEASES (DIS) & 001-139 & 8 & 2.3 & 1.0 & 5.0 \\
\hline -Intestinal Infectious Dis & 001-009 & 5 & 1.6 & 0.6 & 4.4 \\
\hline -Polio \& Other Viral CNS Dis & 045-049 & 1 & 0.2 & 0.0 & 1.6 \\
\hline -Viral Dis with Exanthem & 050-059 & 1 & 0.2 & 0.0 & 1.6 \\
\hline -Other Viral Dis \& Chlamydiae & 070-079 & 1 & 0.2 & 0.0 & 1.6 \\
\hline MALIGNANT NEOPLASMS & $\begin{array}{l}140-208, \\
209.0-209.3, \\
230-234\end{array}$ & 5 & 1.5 & 0.6 & 3.6 \\
\hline -Breast & $174-175$ & 5 & 1.5 & 0.6 & 3.6 \\
\hline BENIGN \& UNCERTAIN NEOPLASMS & $\begin{array}{l}209.4-209.6 \\
210-229,235-239\end{array}$ & 5 & 2.5 & 1.0 & 6.4 \\
\hline ENDOCRINE/METABOLIC/IMMUNITY & 240-279 & 3 & 0.7 & 0.2 & 2.1 \\
\hline -Other Endocrine Gland Dis & $249-259$ & 1 & 0.2 & 0.0 & 1.6 \\
\hline -Other Metabolic \& Immunity Disorders & $270-279$ & 2 & 0.5 & 0.1 & 1.8 \\
\hline MENTAL DISORDERS & 290-319 & 1 & 0.4 & 0.1 & 2.7 \\
\hline -Non-Psychotic Disorders & $300-302,306-316$ & 1 & 0.4 & 0.1 & 2.7 \\
\hline NERVOUS SYSTEM (NS) \& SENSE ORGANS & $320-389$ & 10 & 9.1 & 2.5 & 33.1 \\
\hline -Other Disorders of Central NS & 340-349 & 3 & 7.0 & 1.3 & 36.7 \\
\hline -Disorders of Peripheral NS & $350-359$ & 5 & 1.6 & 0.6 & 4.4 \\
\hline -Disorders of Eye & $360-379$ & 2 & 0.5 & 0.1 & 1.8 \\
\hline CIRCULATORY SYSTEM & $390-459$ & 9 & 2.7 & 1.3 & 5.6 \\
\hline -Hypertensive Dis & $401-405$ & 1 & 0.2 & 0.0 & 1.6 \\
\hline -Dis of Pulmonary Circulation & $415-417$ & 1 & 0.2 & 0.0 & 1.6 \\
\hline -Other Heart Dis & $420-429$ & 3 & 1.2 & 0.3 & 4.3 \\
\hline -Cerebrovascular Dis & $430-438$ & 1 & 0.2 & 0.0 & 1.6 \\
\hline -Dis of Veins, Lymphatics, Other & 451-459 & 3 & 0.8 & 0.3 & 2.7 \\
\hline RESPIRATORY SYSTEM & $460-519$ & 45 & 16.8 & 12.1 & 23.3 \\
\hline -Acute Respiratory Infections & $460-466$ & 16 & 6.0 & 3.5 & 10.3 \\
\hline -Other Dis Upper Respiratory Tract & $470-478$ & 6 & 2.1 & 0.8 & 5.3 \\
\hline -Pneumonia \& Influenza & $480-488$ & 19 & 7.2 & 4.3 & 11.9 \\
\hline -Chronic Obstructive Dis & $490-496$ & 3 & 0.8 & 0.3 & 2.7 \\
\hline -Other Respiratory Dis & $510-519$ & 1 & 0.7 & 0.1 & 5.0 \\
\hline DIGESTIVE SYSTEM & $520-579$ & 25 & 11.6 & 7.5 & 17.9 \\
\hline -Oral Cavity, Saliva Glands, Jaw & \begin{tabular}{|l|}
$520-529$ \\
\end{tabular} & 3 & 1.2 & 0.3 & 4.7 \\
\hline -Esophagus, Stomach, Duodenum & $530-538$ & 2 & 0.5 & 0.1 & 1.8 \\
\hline -Appendicitis & $540-543$ & 4 & 1.8 & 0.7 & 5.1 \\
\hline
\end{tabular}

(Continued)

*Only those diagnostic categories with at least one occurrence appear in this table.

**Standardized to age distribution of 2000 U.S. population. 


\section{Lawrence Livermore National Laboratory 2010}

Absence Data

Appendix K. Age-Adjusted Illness and Injury Rates by Diagnostic Category*

Part 2. Women

\begin{tabular}{|c|c|c|c|c|c|}
\hline & & $\begin{array}{l}\text { Number of } \\
\text { Diagnoses }\end{array}$ & $\begin{array}{c}\text { Age-Adjusted } \\
\text { Rate per } 1,000 * *\end{array}$ & \begin{tabular}{|c|} 
Lower $95 \%$ \\
Confidence \\
Limit per 1,000
\end{tabular} & $\begin{array}{c}\text { Upper } 95 \% \\
\text { Confidence } \\
\text { Limit per 1,000 }\end{array}$ \\
\hline Diagnostic Category & ICD-9-CM Code & & & & \\
\hline -Hernias & $550-553$ & 2 & 1.4 & 0.3 & 5.6 \\
\hline -Enteritis, Colitis & 555-558 & 1 & 0.2 & 0.0 & 1.6 \\
\hline -Other Intestinal Dis & $560-569$ & 5 & 2.4 & 0.9 & 6.1 \\
\hline -Other Digestive Dis & $570-579$ & 8 & 4.0 & 1.9 & 8.7 \\
\hline GENITOURINARY SYSTEM & $580-629$ & 20 & 7.9 & 4.9 & 12.8 \\
\hline -Other Urinary Dis & 590-599 & 4 & 1.1 & 0.4 & 2.9 \\
\hline -Breast Disorders & $610-612$ & 1 & 0.4 & 0.1 & 2.7 \\
\hline -Other Female Disorders & 617-629 & 15 & 6.5 & 3.7 & 11.3 \\
\hline PREGNANCY \& CHILDBIRTH & $630-679$ & 1 & 0.8 & 0.1 & 5.6 \\
\hline -Pregnancy with Abortive Outcome & 634-639 & 1 & 0.8 & 0.1 & 5.6 \\
\hline SKIN AND SUBCUTANEOUS TISSUE & 680-709 & 2 & 0.5 & 0.1 & 1.8 \\
\hline -Infections & $680-686$ & 1 & 0.2 & 0.0 & 1.6 \\
\hline -Other & 700-709 & 1 & 0.2 & 0.0 & 1.6 \\
\hline $\begin{array}{l}\text { MUSCULOSKELETAL \& CONNECTIVE } \\
\text { TISSUE }\end{array}$ & 710-739 & 52 & 19.8 & 14.6 & 26.6 \\
\hline -Arthropathies & 710-719 & 18 & 6.9 & 4.2 & 11.5 \\
\hline -Dorsopathies & 720-724 & 19 & 7.2 & 4.4 & 11.9 \\
\hline -Rheumatism, Excluding Back & 725-729 & 13 & 4.5 & 2.5 & 8.1 \\
\hline -Other Dis \& Acquired Deformities & 730-739 & 2 & 1.1 & 0.3 & 4.6 \\
\hline $\begin{array}{l}\text { SYMPTOMS, SIGNS, \& ILL-DEFINED } \\
\text { CONDITIONS }\end{array}$ & 780-799 & 28 & 12.8 & 8.5 & 19.3 \\
\hline -Symptoms & 780-789 & 28 & 12.8 & 8.5 & 19.3 \\
\hline INJURY \& POISONING & $800-999$ & 25 & 14.0 & 5.9 & 33.2 \\
\hline -Fracture - Upper Limb & $810-819$ & 3 & 0.8 & 0.3 & 2.7 \\
\hline -Fracture - Lower Limb & 820-829 & 5 & 2.2 & 0.8 & 6.0 \\
\hline -Dislocation & 830-839 & 5 & 1.5 & 0.6 & 3.6 \\
\hline -Sprains \& Strains - Other & $840-845,848$ & 7 & 2.1 & 1.0 & 4.4 \\
\hline -Intracranial Injury & $850-854$ & 1 & 0.4 & 0.1 & 2.7 \\
\hline -Open Wound - Head, Neck, Trunk & $870-879$ & 1 & 0.2 & 0.0 & 1.6 \\
\hline -Contusion & 920-924 & 1 & 0.2 & 0.0 & 1.6 \\
\hline -Complications \& Unspecified Injuries & 958-959 & 2 & 6.7 & 1.2 & 38.1 \\
\hline Total & & 239 & 103.2 & 84.3 & 126.4 \\
\hline
\end{tabular}

*Only those diagnostic categories with at least one occurrence appear in this table.

**Standardized to age distribution of 2000 U.S. population. 


\section{Lawrence Livermore National Laboratory 2010}

Absence Data

Appendix K. Age-Adjusted Illness and Injury Rates by Diagnostic Category*

Part 3. Men and Women

\begin{tabular}{|c|c|c|c|c|c|}
\hline & & $\begin{array}{l}\text { Number of } \\
\text { Diagnoses }\end{array}$ & $\begin{array}{c}\text { Age-Adjusted } \\
\text { Rate per } 1,000 * *\end{array}$ & $\begin{array}{c}\text { Lower } 95 \% \\
\text { Confidence } \\
\text { Limit per } 1,000\end{array}$ & $\begin{array}{c}\text { Upper } 95 \% \\
\text { Confidence } \\
\text { Limit per 1,000 }\end{array}$ \\
\hline Diagnostic Category & ICD-9-CM Code & & & & \\
\hline INFECTIOUS \& PARASITIC DISEASES (DIS) & 001-139 & 17 & 2.9 & 1.2 & 7.3 \\
\hline -Intestinal Infectious Dis & 001-009 & 6 & 0.6 & 0.2 & 1.3 \\
\hline -Other Bacterial Dis & 030-041 & 3 & 1.6 & 0.3 & 8.1 \\
\hline -Polio \& Other Viral CNS Dis & 045-049 & 2 & 0.2 & 0.0 & 0.8 \\
\hline -Viral Dis with Exanthem & 050-059 & 2 & 0.1 & 0.0 & 0.5 \\
\hline -Other Viral Dis \& Chlamydiae & 070-079 & 4 & 0.4 & 0.2 & 1.2 \\
\hline MALIGNANT NEOPLASMS & $\begin{array}{l}140-208, \\
209.0-209.3 \\
230-234\end{array}$ & 27 & 2.2 & 1.5 & 3.3 \\
\hline -Digestive \& Peritoneal & $150-159$ & 7 & 0.6 & 0.3 & 1.2 \\
\hline -Respiratory \& Intrathoracic & $160-165$ & 1 & 0.1 & 0.0 & 0.5 \\
\hline -Breast & 174-175 & 5 & 0.5 & 0.2 & 1.2 \\
\hline -Genitourinary & $179-189$ & 6 & 0.5 & 0.2 & 1.1 \\
\hline -Endocrine & $\begin{array}{l}193-194, \\
209.0-209.3\end{array}$ & 2 & 0.1 & 0.0 & 0.5 \\
\hline -Other \& Unspecified Sites & $195-199,209.7$ & 3 & 0.3 & 0.1 & 1.0 \\
\hline -Lymphatic \& Hematopoietic & $200-208$ & 3 & 0.2 & 0.1 & 0.6 \\
\hline BENIGN \& UNCERTAIN NEOPLASMS & $\begin{array}{l}209.4-209.6 \\
210-229,235-239\end{array}$ & 10 & 1.2 & 0.6 & 2.4 \\
\hline ENDOCRINE/METABOLIC/IMMUNITY & $240-279$ & 4 & 0.3 & 0.1 & 0.9 \\
\hline -Other Endocrine Gland Dis & $249-259$ & 1 & 0.1 & 0.0 & 0.5 \\
\hline -Other Metabolic \& Immunity Disorders & 270-279 & 3 & 0.3 & 0.1 & 0.9 \\
\hline BLOOD \& BLOOD-FORMING ORGANS & 280-289 & 1 & 0.1 & 0.0 & 0.5 \\
\hline MENTAL DISORDERS & 290-319 & 6 & 1.1 & 0.5 & 2.6 \\
\hline -Non-Psychotic Disorders & $300-302,306-316$ & 4 & 0.7 & 0.2 & 2.1 \\
\hline -Alcohol Dependence & 303 & 1 & 0.3 & 0.0 & 1.8 \\
\hline -Drug Dependence & $304-305$ & 1 & 0.1 & 0.0 & 0.9 \\
\hline NERVOUS SYSTEM (NS) \& SENSE ORGANS & 320-389 & 31 & 4.7 & 2.5 & 8.6 \\
\hline -Hereditary/Degenerative Central NS Dis & 330-337 & 1 & 0.1 & 0.0 & 0.5 \\
\hline -Other Disorders of Central NS & $340-349$ & 3 & 1.7 & 0.4 & 7.9 \\
\hline -Disorders of Peripheral NS & 350-359 & 10 & 0.9 & 0.5 & 1.7 \\
\hline -Disorders of Eye & $360-379$ & 13 & 1.4 & 0.8 & 2.4 \\
\hline -Diseases of Ear \& Mastoid & 380-389 & 4 & 0.6 & 0.2 & 1.8 \\
\hline CIRCULATORY SYSTEM & $390-459$ & 30 & 3.0 & 2.0 & 4.4 \\
\hline -Hypertensive Dis & $401-405$ & 1 & 0.1 & 0.0 & 0.5 \\
\hline -Ischemic Heart Dis & $410-414$ & 6 & 0.6 & 0.3 & 1.5 \\
\hline -Dis of Pulmonary Circulation & $415-417$ & 1 & 0.1 & 0.0 & 0.5 \\
\hline
\end{tabular}

(Continued)

* Only those diagnostic categories with at least one occurrence appear in this table.

**Standardized to age distribution of 2000 U.S. population. 


\section{Lawrence Livermore National Laboratory 2010}

\section{Absence Data}

Appendix K. Age-Adjusted IIIness and Injury Rates by Diagnostic Category*

Part 3. Men and Women

\begin{tabular}{|c|c|c|c|c|c|}
\hline & & $\begin{array}{l}\text { Number of } \\
\text { Diagnoses }\end{array}$ & $\begin{array}{l}\text { Age-Adjusted } \\
\text { Rate per } 1,000 * *\end{array}$ & $\begin{array}{c}\text { Lower } 95 \% \\
\text { Confidence } \\
\text { Limit per 1,000 }\end{array}$ & $\begin{array}{c}\text { Upper } 95 \% \\
\text { Confidence } \\
\text { Limit per 1,000 }\end{array}$ \\
\hline Diagnostic Category & ICD-9-CM Code & \multirow[b]{2}{*}{8} & \multirow[b]{2}{*}{0.8} & \multirow[b]{2}{*}{0.4} & \multirow[b]{2}{*}{1.6} \\
\hline -Other Heart Dis & $420-429$ & & & & \\
\hline -Cerebrovascular Dis & $430-438$ & 5 & 0.5 & 0.2 & 1.2 \\
\hline -Dis of Arteries \& Capillaries & $440-449$ & 3 & 0.3 & 0.1 & 0.9 \\
\hline -Dis of Veins, Lymphatics, Other & $451-459$ & 6 & 0.7 & 0.3 & 1.7 \\
\hline RESPIRATORY SYSTEM & $460-519$ & 109 & 16.2 & 11.8 & 22.2 \\
\hline -Acute Respiratory Infections & $460-466$ & 41 & 5.1 & 3.6 & 7.1 \\
\hline -Other Dis Upper Respiratory Tract & $470-478$ & 14 & 1.5 & 0.8 & 2.8 \\
\hline -Pneumonia \& Influenza & $480-488$ & 46 & 8.8 & 5.1 & 15.0 \\
\hline -Chronic Obstructive Dis & $490-496$ & 7 & 0.7 & 0.3 & 1.4 \\
\hline -Other Respiratory Dis & $510-519$ & 1 & 0.1 & 0.0 & 1.0 \\
\hline DIGESTIVE SYSTEM & $520-579$ & 66 & 7.9 & 6.1 & 10.3 \\
\hline -Oral Cavity, Saliva Glands, Jaw & $520-529$ & 6 & 0.8 & 0.3 & 2.1 \\
\hline -Esophagus, Stomach, Duodenum & $530-538$ & 6 & 0.5 & 0.2 & 1.3 \\
\hline -Appendicitis & $540-543$ & 8 & 0.8 & 0.4 & 1.7 \\
\hline -Hernias & $550-553$ & 19 & 2.5 & 1.5 & 4.1 \\
\hline -Enteritis, Colitis & $555-558$ & 1 & 0.1 & 0.0 & 0.5 \\
\hline -Other Intestinal Dis & $560-569$ & 9 & 1.1 & 0.5 & 2.2 \\
\hline -Other Digestive Dis & $570-579$ & 17 & 2.1 & 1.2 & 3.5 \\
\hline GENITOURINARY SYSTEM & $580-629$ & 30 & 3.9 & 2.7 & $\overline{5.8}$ \\
\hline -Other Urinary Dis & $590-599$ & 10 & 1.2 & 0.6 & 2.2 \\
\hline -Male Genital Organ Dis & $600-608$ & 4 & 0.5 & 0.2 & 1.4 \\
\hline -Breast Disorders & $610-612$ & 1 & 0.1 & 0.0 & 0.9 \\
\hline -Other Female Disorders & $617-629$ & 15 & 2.1 & 1.2 & 3.7 \\
\hline PREGNANCY \& CHILDBIRTH & $630-679$ & 1 & 0.3 & 0.0 & 1.8 \\
\hline -Pregnancy with Abortive Outcome & $634-639$ & 1 & 0.3 & 0.0 & 1.8 \\
\hline SKIN AND SUBCUTANEOUS TISSUE & 680-709 & 5 & 0.5 & 0.2 & 1.2 \\
\hline -Infections & $680-686$ & 2 & 0.2 & 0.0 & 0.8 \\
\hline -Other & 700-709 & 3 & 0.3 & 0.1 & 1.0 \\
\hline $\begin{array}{l}\text { MUSCULOSKELETAL \& CONNECTIVE } \\
\text { TISSUE }\end{array}$ & 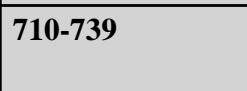 & 121 & 13.9 & 10.8 & 17.9 \\
\hline -Arthropathies & 710-719 & 52 & 6.5 & 4.2 & 10.3 \\
\hline -Dorsopathies & $720-724$ & 42 & 4.5 & 3.2 & 6.2 \\
\hline -Rheumatism, Excluding Back & 725-729 & 24 & 2.5 & 1.6 & 3.9 \\
\hline -Other Dis \& Acquired Deformities & 730-739 & 3 & 0.3 & 0.1 & 1.1 \\
\hline $\begin{array}{l}\text { SYMPTOMS, SIGNS, \& ILL-DEFINED } \\
\text { CONDITIONS }\end{array}$ & \begin{tabular}{|l|}
$780-799$ \\
\end{tabular} & 55 & 6.4 & 4.8 & 8.5 \\
\hline
\end{tabular}

(Continued)

*Only those diagnostic categories with at least one occurrence appear in this table.

**Standardized to age distribution of 2000 U.S. population. 
Lawrence Livermore National Laboratory 2010

Absence Data

Appendix K. Age-Adjusted Illness and Injury Rates by Diagnostic Category*

Part 3. Men and Women

\begin{tabular}{|c|c|c|c|c|c|}
\hline & & $\begin{array}{c}\text { Number of } \\
\text { Diagnoses }\end{array}$ & $\begin{array}{c}\text { Age-Adjusted } \\
\text { Rate per } 1,000^{* *}\end{array}$ & $\begin{array}{c}\text { Lower } 95 \% \\
\text { Confidence } \\
\text { Limit per 1,000 }\end{array}$ & $\begin{array}{c}\text { Upper } 95 \% \\
\text { Confidence } \\
\text { Limit per 1,000 }\end{array}$ \\
\hline Diagnostic Category & ICD-9-CM Code & \multirow[b]{2}{*}{55} & \multirow[b]{2}{*}{6.4} & \multirow[b]{2}{*}{4.8} & \multirow[b]{2}{*}{8.5} \\
\hline -Symptoms & 780-789 & & & & \\
\hline INJURY \& POISONING & 800-999 & 84 & 14.5 & 9.9 & 21.3 \\
\hline -Fracture - Upper Limb & 810-819 & 8 & 0.9 & 0.4 & 2.0 \\
\hline -Fracture - Lower Limb & 820-829 & 11 & 1.4 & 0.7 & 2.6 \\
\hline -Dislocation & 830-839 & 10 & 1.3 & 0.6 & 2.5 \\
\hline -Sprains \& Strains - Back & 846-847 & 5 & 0.5 & 0.2 & 1.3 \\
\hline -Sprains \& Strains - Other & $840-845,848$ & 34 & 7.7 & 4.2 & 14.1 \\
\hline -Intracranial Injury & $850-854$ & 1 & 0.1 & 0.0 & 0.9 \\
\hline -Open Wound - Head, Neck, Trunk & 870-879 & 3 & 0.2 & 0.1 & 0.6 \\
\hline -Superficial Injury & 910-919 & 2 & 0.1 & 0.0 & 0.5 \\
\hline -Contusion & $920-924$ & 5 & 0.3 & 0.1 & 0.8 \\
\hline -Complications \& Unspecified Injuries & 958-959 & 5 & 1.9 & 0.5 & 7.6 \\
\hline Total & & 597 & 79.0 & 69.6 & 89.6 \\
\hline
\end{tabular}

* Only those diagnostic categories with at least one occurrence appear in this table.

**Standardized to age distribution of 2000 U.S. population. 
Lawrence Livermore National Laboratory 2010

OSHA Data

Appendix L. Number of Workers with at Least One OSHA Event by Gender, Age, and Job Category*

\begin{tabular}{|c|c|c|c|c|c|c|c|c|c|c|}
\hline \multirow{3}{*}{ Job Category } & \multicolumn{5}{|c|}{ Women } & \multicolumn{4}{|c|}{ Men } & \multirow[b]{3}{*}{ TOTAL } \\
\hline & \multicolumn{4}{|c|}{ Age Group } & \multirow[b]{2}{*}{ TOTAL } & \multicolumn{3}{|c|}{ Age Group } & \multirow[b]{2}{*}{ TOTAL } & \\
\hline & $16-29$ & $30-39$ & $40-49$ & $\mathbf{5 0 +}$ & & $30-39$ & $40-49$ & $50+$ & & \\
\hline Professional & 1 & 3 & 9 & 14 & 27 & 1 & 5 & 11 & 17 & 44 \\
\hline Technical Support & 0 & 2 & 0 & 1 & 3 & 0 & 3 & 19 & 22 & 25 \\
\hline Service & 0 & 0 & 0 & 1 & 1 & 0 & 0 & 1 & 1 & 2 \\
\hline Crafts & 0 & 0 & 0 & 0 & 0 & 0 & 2 & 2 & 4 & 4 \\
\hline TOTAL & 1 & 5 & 9 & 16 & 31 & 1 & 10 & 33 & 44 & 75 \\
\hline
\end{tabular}

*Only those job categories and gender/age combinations with at least one OSHA event appear in this table.

Appendix M. Total Number of Workdays Lost or with Restricted Activity from OSHA Events by Gender and Age

\begin{tabular}{|c|c|c|c|c|c|c|c|c|c|}
\hline \multirow[b]{2}{*}{ Age Group } & \multicolumn{3}{|c|}{ Women } & \multicolumn{3}{|c|}{ Men } & \multicolumn{3}{|c|}{ TOTAL } \\
\hline & $\begin{array}{c}\text { Number of } \\
\text { Events }\end{array}$ & \begin{tabular}{c|} 
Days \\
Restricted
\end{tabular} & Days Lost & $\begin{array}{c}\text { Number of } \\
\text { Events }\end{array}$ & $\begin{array}{c}\text { Days } \\
\text { Restricted }\end{array}$ & Days Lost & $\begin{array}{c}\text { Number of } \\
\text { Events }\end{array}$ & $\begin{array}{c}\text { Days } \\
\text { Restricted }\end{array}$ & Days Lost \\
\hline $16-29$ & 1 & 0 & 0 & 0 & 0 & 0 & 1 & 0 & 0 \\
\hline 30 - 39 & 5 & 0 & 0 & 1 & 54 & 0 & 6 & 54 & 0 \\
\hline $40-49$ & 9 & 25 & 3 & 10 & 178 & 0 & 19 & 203 & 3 \\
\hline $50+$ & 16 & 239 & 271 & 33 & 368 & 178 & 49 & 607 & 449 \\
\hline TOTAL & 31 & 264 & 274 & 44 & 600 & 178 & 75 & 864 & 452 \\
\hline
\end{tabular}

Appendix N. Total Number of Workdays Lost or with Restricted Activity from OSHA Events by Gender and Job Category*

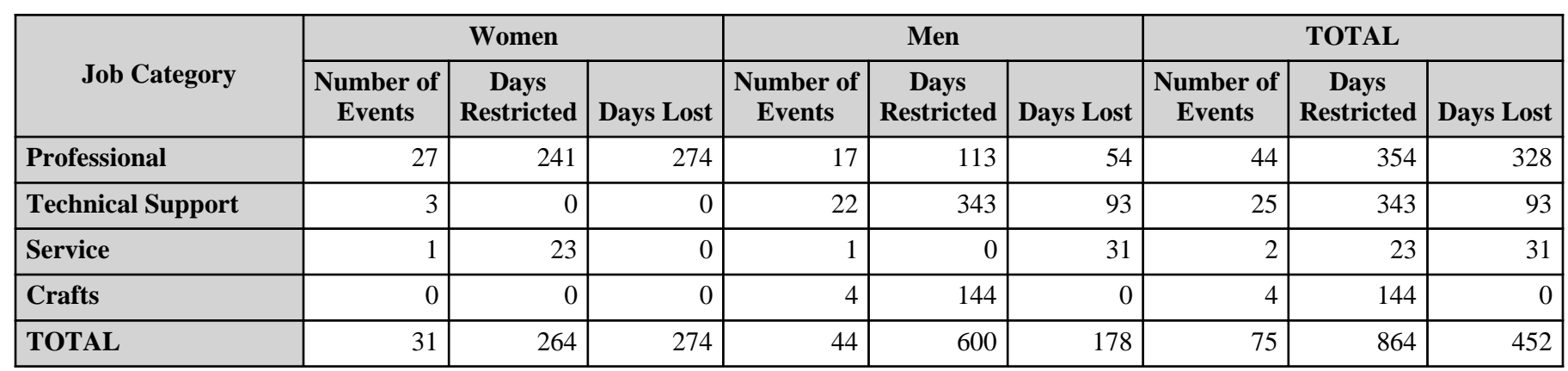

*Only those job categories with at least one OSHA event appear in this table. 
Lawrence Livermore National Laboratory 2010

OSHA Data

Appendix O. Number of Diagnoses in Each Diagnostic Category by Gender and Age*

\begin{tabular}{|c|c|c|c|c|c|c|}
\hline & & \multicolumn{5}{|c|}{ Women } \\
\hline & & \multicolumn{4}{|c|}{ Age Group } & \multirow[b]{2}{*}{ TOTAL } \\
\hline & & $16-29$ & 30 - 39 & $40-49$ & $\mathbf{5 0 +}$ & \\
\hline Diagnostic Category & ICD-9-CM code & \multirow[b]{2}{*}{0} & \multirow[b]{2}{*}{0} & \multirow[b]{2}{*}{1} & \multirow[b]{2}{*}{2} & \multirow[b]{2}{*}{3} \\
\hline NERVOUS SYSTEM (NS) \& SENSE ORGANS & $320-389$ & & & & & \\
\hline -Disorders of Peripheral NS & 350-359 & 0 & 0 & 1 & 2 & 3 \\
\hline -Diseases of Ear \& Mastoid & 380-389 & 0 & 0 & 0 & 0 & 0 \\
\hline SKIN AND SUBCUTANEOUS TISSUE & 680-709 & 0 & 0 & 0 & 0 & 0 \\
\hline -Other Inflammatory Conditions & 690-698 & 0 & 0 & 0 & 0 & 0 \\
\hline $\begin{array}{l}\text { MUSCULOSKELETAL \& CONNECTIVE } \\
\text { TISSUE }\end{array}$ & 710-739 & 0 & 2 & 10 & 13 & 25 \\
\hline -Arthropathies & 710-719 & 0 & 1 & 8 & 5 & 14 \\
\hline -Dorsopathies & $720-724$ & 0 & 0 & 0 & 2 & 2 \\
\hline -Rheumatism, Excluding Back & 725-729 & 0 & 1 & 2 & 6 & 9 \\
\hline $\begin{array}{l}\text { SYMPTOMS, SIGNS, \& ILL-DEFINED } \\
\text { CONDITIONS }\end{array}$ & $780-799$ & 0 & 1 & 1 & 4 & 6 \\
\hline -Symptoms & 780-789 & 0 & 1 & 1 & 4 & 6 \\
\hline INJURY \& POISONING & $800-999$ & 1 & 9 & 9 & 12 & 31 \\
\hline -Fracture - Lower Limb & $820-829$ & 0 & 0 & 0 & 2 & 2 \\
\hline -Sprains \& Strains - Back & 846-847 & 0 & 0 & 1 & 0 & 1 \\
\hline -Sprains \& Strains - Other & $840-845,848$ & 0 & 0 & 2 & 2 & 4 \\
\hline -Open Wound - Upper Limb & $880-887$ & 0 & 0 & 0 & 0 & 0 \\
\hline -Open Wound - Lower Limb & 890-897 & 0 & 1 & 0 & 0 & 1 \\
\hline -Superficial Injury & 910-919 & 0 & 1 & 0 & 0 & 1 \\
\hline -Contusion & $920-924$ & 0 & 2 & 0 & 1 & 3 \\
\hline -Burns & 940-949 & 1 & 0 & 0 & 0 & 1 \\
\hline -Complications \& Unspecified Injuries & 958-959 & 0 & 5 & 6 & 6 & 17 \\
\hline -Poisoning - Medicinal/Biological & 960-979 & 0 & 0 & 0 & 0 & 0 \\
\hline -Toxic Effects - Non-medicinal & 980-989 & 0 & 0 & 0 & 1 & 1 \\
\hline
\end{tabular}

*Only those diagnostic categories and gender/age combinations with at least one OSHA event appear in this table. 
Lawrence Livermore National Laboratory 2010

OSHA Data

Appendix O. Number of Diagnoses in Each Diagnostic Category by Gender and Age*

\begin{tabular}{|c|c|c|c|c|c|c|}
\hline & & \multicolumn{4}{|c|}{ Men } & \multirow[b]{3}{*}{ TOTAL } \\
\hline & & \multicolumn{3}{|c|}{ Age Group } & \multirow[b]{2}{*}{ TOTAL } & \\
\hline & & $30-39$ & $40-49$ & $50+$ & & \\
\hline Diagnostic Category & ICD-9-CM code & & & & & \\
\hline NERVOUS SYSTEM (NS) \& SENSE ORGANS & 320-389 & 0 & 2 & 9 & 11 & 14 \\
\hline -Disorders of Peripheral NS & 350-359 & 0 & 0 & 1 & 1 & 4 \\
\hline -Diseases of Ear \& Mastoid & $380-389$ & 0 & 2 & 8 & 10 & 10 \\
\hline SKIN AND SUBCUTANEOUS TISSUE & $680-709$ & 0 & 1 & 2 & 3 & 3 \\
\hline -Other Inflammatory Conditions & 690-698 & 0 & 1 & 2 & 3 & 3 \\
\hline $\begin{array}{l}\text { MUSCULOSKELETAL \& CONNECTIVE } \\
\text { TISSUE }\end{array}$ & \begin{tabular}{|l|}
$710-739$ \\
\end{tabular} & 1 & 7 & 19 & 27 & 52 \\
\hline -Arthropathies & $710-719$ & 0 & 3 & 14 & 17 & 31 \\
\hline -Dorsopathies & $720-724$ & 1 & 0 & 5 & 6 & 8 \\
\hline -Rheumatism, Excluding Back & 725-729 & 0 & 4 & 0 & 4 & 13 \\
\hline $\begin{array}{l}\text { SYMPTOMS, SIGNS, \& ILL-DEFINED } \\
\text { CONDITIONS }\end{array}$ & 780-799 & 0 & 0 & 2 & 2 & 8 \\
\hline -Symptoms & 780-789 & 0 & 0 & 2 & 2 & 8 \\
\hline INJURY \& POISONING & $800-999$ & 1 & 6 & 23 & 30 & 61 \\
\hline -Fracture - Lower Limb & $820-829$ & 0 & 0 & 1 & 1 & 3 \\
\hline -Sprains \& Strains - Back & 846-847 & 1 & 0 & 4 & 5 & 6 \\
\hline -Sprains \& Strains - Other & $840-845,848$ & 0 & 1 & 6 & 7 & 11 \\
\hline -Open Wound - Upper Limb & 880-887 & 0 & 1 & 3 & 4 & 4 \\
\hline -Open Wound - Lower Limb & $890-897$ & 0 & 0 & 0 & 0 & 1 \\
\hline -Superficial Injury & 910-919 & 0 & 0 & 0 & 0 & 1 \\
\hline -Contusion & 920-924 & 0 & 0 & 1 & 1 & 4 \\
\hline -Burns & 940-949 & 0 & 0 & 0 & 0 & 1 \\
\hline -Complications \& Unspecified Injuries & 958-959 & 0 & 2 & 8 & 10 & 27 \\
\hline -Poisoning - Medicinal/Biological & 960-979 & 0 & 1 & 0 & 1 & 1 \\
\hline -Toxic Effects - Non-medicinal & 980-989 & 0 & 1 & 0 & 1 & 2 \\
\hline
\end{tabular}

\begin{tabular}{|c|c|c|c|c|c|c|c|c|c|c|}
\hline & \multicolumn{5}{|c|}{ Women } & \multicolumn{4}{|c|}{ Men } & \multirow[b]{3}{*}{ TOTAI } \\
\hline & \multicolumn{4}{|c|}{ Age Group } & \multirow[b]{2}{*}{ TOTAL } & \multicolumn{3}{|c|}{ Age Group } & \multirow[b]{2}{*}{ TOTAL } & \\
\hline & $16-29$ & 30 - 39 & 40 - 49 & $50+$ & & 30 - 39 & $40-49$ & $50+$ & & \\
\hline Diagnostic Category & & & & & & & & & & \\
\hline Total & 1 & 12 & 21 & 31 & 65 & 2 & 16 & 55 & 73 & 138 \\
\hline
\end{tabular}

*Only those diagnostic categories and gender/age combinations with at least one OSHA event appear in this table. 


\section{Lawrence Livermore National Laboratory 2010}

OSHA Data

Appendix P. Number of Workdays Lost or with Restricted Activity in Each Diagnostic Category by Gender and Age*

\begin{tabular}{|c|c|c|c|c|c|c|c|c|c|}
\hline & & \multicolumn{8}{|c|}{ Women } \\
\hline & & \multicolumn{8}{|c|}{ Age Group } \\
\hline & & \multicolumn{2}{|c|}{$16-29$} & \multicolumn{2}{|c|}{30 - 39} & \multicolumn{2}{|c|}{$40-49$} & \multicolumn{2}{|c|}{$50+$} \\
\hline & & $\begin{array}{c}\text { Days } \\
\text { Restricted }\end{array}$ & $\begin{array}{l}\text { Days } \\
\text { Lost }\end{array}$ & $\begin{array}{c}\text { Days } \\
\text { Restricted }\end{array}$ & $\begin{array}{l}\text { Days } \\
\text { Lost }\end{array}$ & $\begin{array}{c}\text { Days } \\
\text { Restricted }\end{array}$ & $\begin{array}{l}\text { Days } \\
\text { Lost }\end{array}$ & \begin{tabular}{c|} 
Days \\
Restricted
\end{tabular} & $\begin{array}{l}\text { Days } \\
\text { Lost }\end{array}$ \\
\hline Diagnostic Category & ICD-9-CM Codes & & & & & & & & \\
\hline -Disorders of Peripheral NS & $350-359$ & 0 & 0 & 0 & 0 & 0 & 0 & 39 & 94 \\
\hline -Arthropathies & $710-719$ & 0 & 0 & 0 & 0 & 25 & 0 & 193 & 115 \\
\hline -Dorsopathies & $720-724$ & 0 & 0 & 0 & 0 & 0 & 0 & 0 & 0 \\
\hline -Rheumatism, Excluding Back & $725-729$ & 0 & 0 & 0 & 0 & 0 & 0 & 0 & 105 \\
\hline -Symptoms & 780-789 & 0 & 0 & 0 & 0 & 0 & 0 & 0 & 11 \\
\hline -Fracture - Lower Limb & $820-829$ & 0 & 0 & 0 & 0 & 0 & 0 & 0 & 1 \\
\hline -Sprains \& Strains - Back & 846-847 & 0 & 0 & 0 & 0 & 0 & 0 & 0 & 0 \\
\hline -Sprains \& Strains - Other & $840-845,848$ & 0 & 0 & 0 & 0 & 0 & 3 & 170 & 10 \\
\hline -Open Wound - Lower Limb & 890-897 & 0 & 0 & 0 & 0 & 0 & 0 & 0 & $\overline{0}$ \\
\hline -Superficial Injury & $910-919$ & 0 & 0 & 0 & 0 & 0 & 0 & 0 & 0 \\
\hline -Contusion & $920-924$ & 0 & 0 & 0 & 0 & 0 & 0 & 0 & 0 \\
\hline -Burns & $940-949$ & 0 & 0 & 0 & 0 & 0 & 0 & 0 & 0 \\
\hline $\begin{array}{l}\text {-Complications \& Unspecified } \\
\text { Injuries }\end{array}$ & 958-959 & 0 & 0 & 0 & 0 & 25 & 0 & 30 & 50 \\
\hline -Toxic Effects - Non-medicinal & $980-989$ & 0 & 0 & 0 & 0 & 0 & 0 & 0 & 11 \\
\hline
\end{tabular}

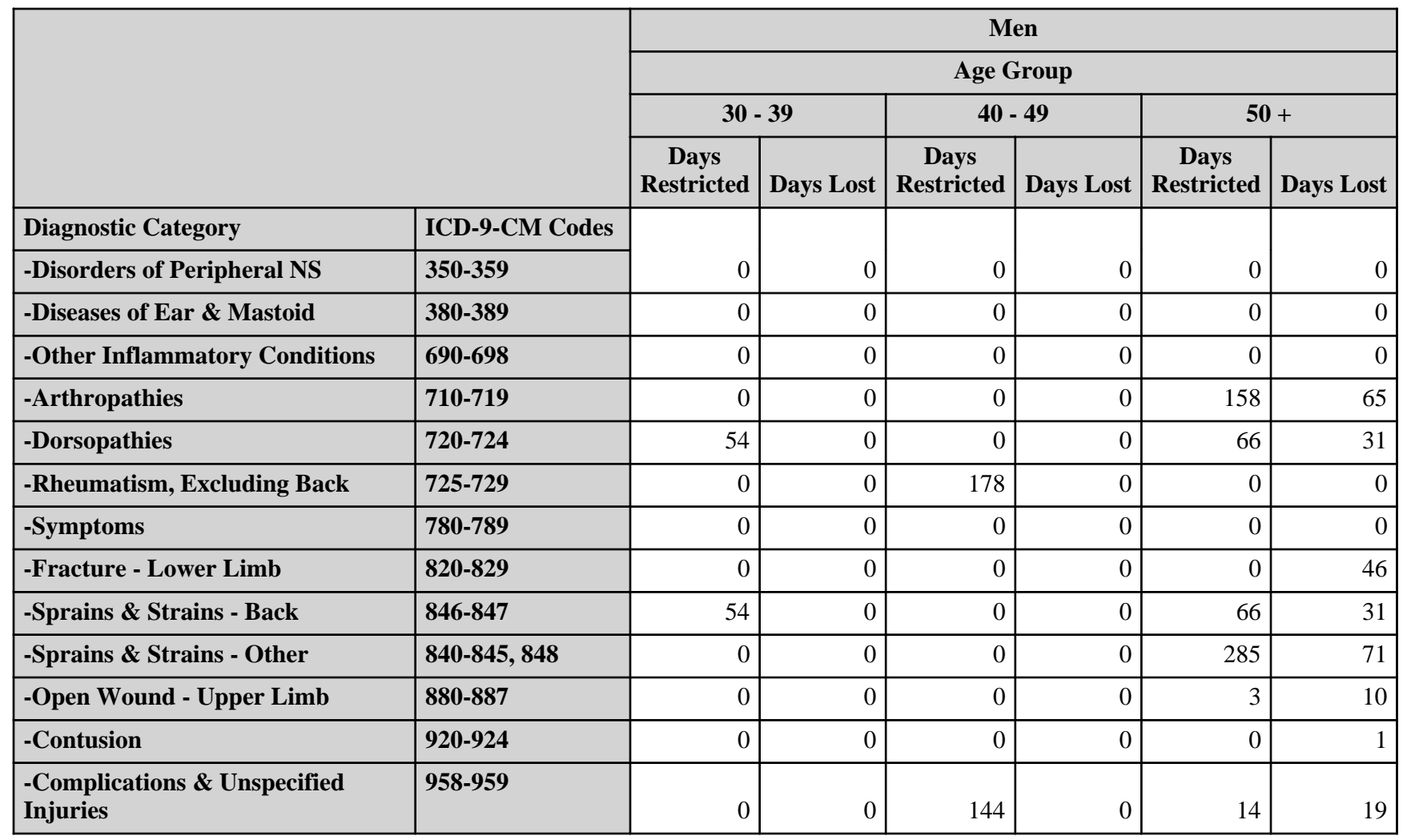

(Continued)

*OSHA events with >1 ICD-9-CM code in the same diagnostic category were counted only once. Only those diagnostic categories and gender/age combinations with at least one occurrence appear in this table. 
Lawrence Livermore National Laboratory 2010

OSHA Data

Appendix P. Number of Workdays Lost or with Restricted Activity in Each Diagnostic Category by Gender and Age*

\begin{tabular}{|c|c|c|c|c|c|c|c|}
\hline & & \multicolumn{6}{|c|}{ Men } \\
\hline & & \multicolumn{6}{|c|}{ Age Group } \\
\hline & & \multicolumn{2}{|c|}{$30-39$} & \multicolumn{2}{|c|}{$40-49$} & \multicolumn{2}{|c|}{$50+$} \\
\hline & & $\begin{array}{c}\text { Days } \\
\text { Restricted }\end{array}$ & Days Lost & $\begin{array}{c}\text { Days } \\
\text { Restricted }\end{array}$ & Days Lost & $\begin{array}{c}\text { Days } \\
\text { Restricted }\end{array}$ & Days Lost \\
\hline Diagnostic Category & ICD-9-CM Codes & \multirow[b]{2}{*}{0} & \multirow[b]{2}{*}{0} & \multirow[b]{2}{*}{0} & \multirow[b]{2}{*}{0} & \multirow[b]{2}{*}{0} & \multirow[b]{2}{*}{0} \\
\hline -Poisoning - Medicinal/Biological & $960-979$ & & & & & & \\
\hline -Toxic Effects - Non-medicinal & 980-989 & 0 & 0 & 0 & 0 & 0 & 0 \\
\hline
\end{tabular}

*OSHA events with >1 ICD-9-CM code in the same diagnostic category were counted only once. Only those diagnostic categories and gender/age combinations with at least one occurrence appear in this table. 
Lawrence Livermore National Laboratory 2010

OSHA Data

Appendix Q. Number of Occurrences in Each Accident Category by Gender and Age*

\begin{tabular}{|c|c|c|c|c|c|c|c|c|c|c|c|}
\hline & & \multicolumn{5}{|c|}{ Women } & \multicolumn{4}{|c|}{ Men } & \multirow[b]{3}{*}{ TOTAL } \\
\hline & & \multicolumn{4}{|c|}{ Age Group } & \multirow[b]{2}{*}{ TOTAL } & \multicolumn{3}{|c|}{ Age Group } & \multirow[b]{2}{*}{ TOTAL } & \\
\hline & & $16-29$ & 30 - 39 & $40-49$ & $\begin{array}{c}50 \\
+\end{array}$ & & 30 - 39 & $40-49$ & $\begin{array}{c}50 \\
+\end{array}$ & & \\
\hline Type of Accident & E Codes & & & & & & & & & & \\
\hline Other Road Vehicle & E826-E829 & 0 & 1 & 0 & 0 & 1 & 0 & 0 & 1 & 1 & 2 \\
\hline $\begin{array}{l}\text { Accidental } \\
\text { Poisoning - Medicinal/Biological }\end{array}$ & E850-E858 & 0 & 0 & 0 & 0 & 0 & 0 & 1 & 0 & 1 & 1 \\
\hline Accidental Poisoning - Non-medicinal & E860-E869 & 0 & 0 & 0 & 1 & 1 & 0 & 0 & 0 & 0 & 1 \\
\hline Falls & E880-E888 & 0 & 1 & 3 & 2 & 6 & 0 & 0 & 3 & 3 & 9 \\
\hline Natural/Environmental Factors & E900-E909 & 0 & 0 & 0 & 0 & 0 & 0 & 1 & 0 & 1 & 1 \\
\hline Other Accidents & E916-E928 & 1 & 3 & 6 & 13 & 23 & 1 & 7 & 27 & 35 & 58 \\
\hline
\end{tabular}

*Only those accident types and gender/age combinations with at least one occurrence appear in this table. 
Lawrence Livermore National Laboratory 2010

OSHA Data

Appendix R. Number of Workdays Lost or with Restricted Activity in Each Accident Category by Gender and Age*

\begin{tabular}{|c|c|c|c|c|c|c|c|c|c|}
\hline & & \multicolumn{8}{|c|}{ Women } \\
\hline & & \multicolumn{8}{|c|}{ Age Group } \\
\hline & & \multicolumn{2}{|c|}{$16-29$} & \multicolumn{2}{|c|}{30 - 39} & \multicolumn{2}{|c|}{$40-49$} & \multicolumn{2}{|c|}{$\mathbf{5 0 +}$} \\
\hline & & $\begin{array}{c}\text { Days } \\
\text { Restricted }\end{array}$ & $\begin{array}{l}\text { Days } \\
\text { Lost }\end{array}$ & $\begin{array}{c}\text { Days } \\
\text { Restricted }\end{array}$ & $\begin{array}{l}\text { Days } \\
\text { Lost }\end{array}$ & $\begin{array}{c}\text { Days } \\
\text { Restricted }\end{array}$ & $\begin{array}{l}\text { Days } \\
\text { Lost }\end{array}$ & $\begin{array}{c}\text { Days } \\
\text { Restricted }\end{array}$ & $\begin{array}{l}\text { Days } \\
\text { Lost }\end{array}$ \\
\hline Type of Accident & E Codes & \multirow[b]{2}{*}{0} & \multirow[b]{2}{*}{0} & \multirow[b]{2}{*}{0} & \multirow[b]{2}{*}{0} & \multirow[b]{2}{*}{0} & \multirow[b]{2}{*}{0} & \multirow[b]{2}{*}{0} & \multirow[b]{2}{*}{0} \\
\hline Other Road Vehicle & E826-E829 & & & & & & & & \\
\hline Accidental Poisoning - Non-medicinal & E860-E869 & 0 & 0 & 0 & 0 & 0 & 0 & 0 & 11 \\
\hline Falls & E880-E888 & 0 & 0 & 0 & 0 & 0 & 3 & 0 & 0 \\
\hline Other Accidents & E916-E928 & 0 & 0 & 0 & 0 & 25 & 0 & 239 & 260 \\
\hline
\end{tabular}

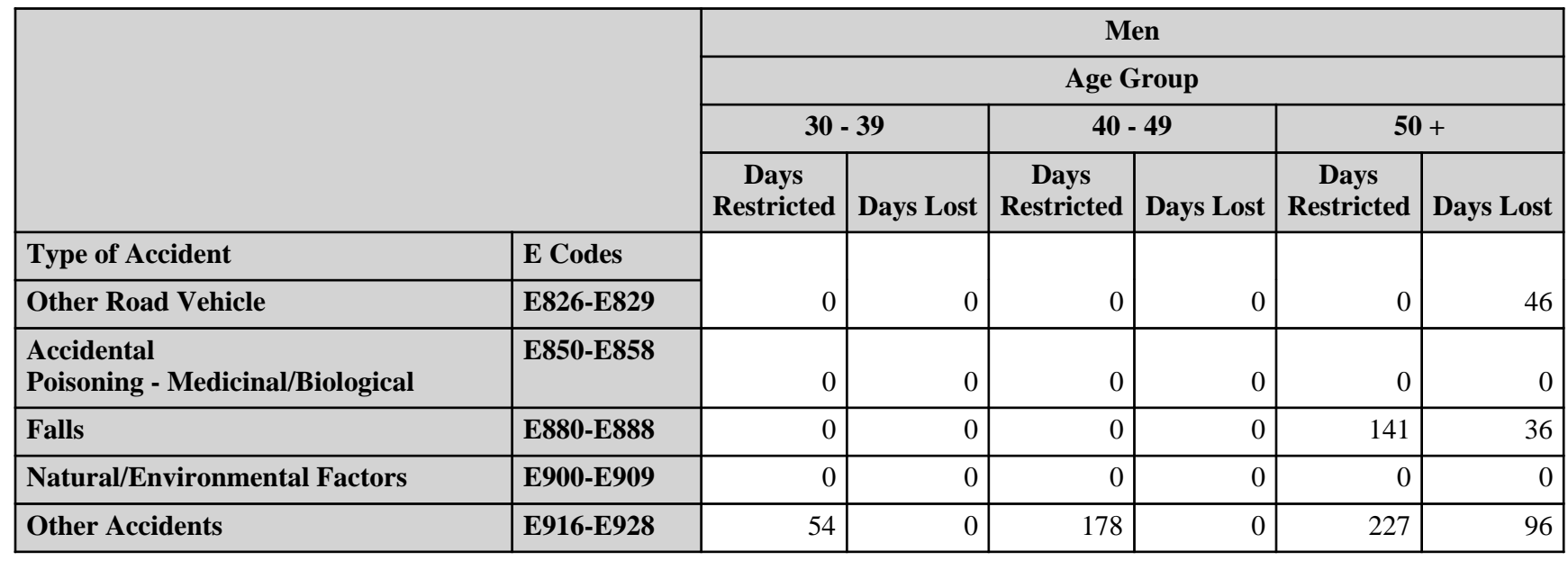

*OSHA events with $>1 \mathrm{E}$ code in the same accident type were counted only once. Only those accident types and gender/age combinations with at least one occurrence appear in this table. 


\section{Lawrence Livermore National Laboratory 2010}

OSHA Data

Appendix S. Number of Diagnoses in Each Diagnostic Category by Gender and Job Category*

\begin{tabular}{|c|c|c|c|c|c|}
\hline & \multicolumn{4}{|c|}{ Women } \\
\hline & & \multicolumn{3}{|c|}{ Job Category } & \multirow[b]{2}{*}{ TOTAL } \\
\hline & & Professional & Technical Support & Service & \\
\hline Diagnostic Category & ICD-9-CM Code & & & & \\
\hline $\begin{array}{l}\text { NERVOUS SYSTEM } \\
\text { (NS) \& SENSE ORGANS }\end{array}$ & 320-389 & 3 & 0 & 0 & 3 \\
\hline $\begin{array}{l}\text {-Disorders of Peripheral } \\
\text { NS }\end{array}$ & $350-359$ & 3 & 0 & 0 & 3 \\
\hline $\begin{array}{l}\text { MUSCULOSKELETAL } \\
\text { \& CONNECTIVE } \\
\text { TISSUE }\end{array}$ & 710-739 & 22 & 2 & 1 & 25 \\
\hline -Arthropathies & $710-719$ & 12 & 1 & 1 & 14 \\
\hline -Dorsopathies & $720-724$ & 2 & 0 & 0 & 2 \\
\hline $\begin{array}{l}\text {-Rheumatism, Excluding } \\
\text { Back }\end{array}$ & 725-729 & 8 & 1 & 0 & 9 \\
\hline $\begin{array}{l}\text { SYMPTOMS, } \\
\text { SIGNS, \& ILL-DEFINED } \\
\text { CONDITIONS }\end{array}$ & 780-799 & 5 & 1 & 0 & 6 \\
\hline -Symptoms & 780-789 & 5 & 1 & 0 & 6 \\
\hline INJURY \& POISONING & $800-999$ & 27 & 3 & 1 & 31 \\
\hline -Fracture - Lower Limb & 820-829 & 2 & 0 & 0 & 2 \\
\hline -Sprains \& Strains - Back & 846-847 & 1 & 0 & 0 & 1 \\
\hline $\begin{array}{l}\text {-Sprains \& Strains - } \\
\text { Other }\end{array}$ & $840-845,848$ & 4 & 0 & 0 & 4 \\
\hline $\begin{array}{l}\text {-Open Wound - Lower } \\
\text { Limb }\end{array}$ & 890-897 & 1 & 0 & 0 & 1 \\
\hline -Superficial Injury & 910-919 & 1 & 0 & 0 & 1 \\
\hline -Contusion & $920-924$ & 3 & 0 & 0 & 3 \\
\hline -Burns & $940-949$ & 1 & 0 & 0 & 1 \\
\hline $\begin{array}{l}\text {-Complications \& } \\
\text { Unspecified Injuries }\end{array}$ & 958-959 & 13 & 3 & 1 & 17 \\
\hline $\begin{array}{l}\text {-Toxic } \\
\text { Effects - Non-medicinal }\end{array}$ & 980-989 & 1 & 0 & 0 & 1 \\
\hline
\end{tabular}

\begin{tabular}{|l|r|r|r|r|}
\hline \multirow{4}{*}{} & \multicolumn{3}{|c|}{ Women } & \multirow{3}{*}{ Job Category } \\
\cline { 2 - 5 } & \multicolumn{3}{|c|}{ TOTAL } \\
\cline { 2 - 5 } & Professional & Technical Support & Service & TOT \\
\cline { 1 - 5 } Diagnostic Category & & & & \\
\cline { 1 - 5 } Total & 57 & & 65 \\
\hline
\end{tabular}

*Only those diagnostic categories and gender/job category combinations with at least one occurrence appear in this table. 


\section{Lawrence Livermore National Laboratory 2010}

OSHA Data

Appendix S. Number of Diagnoses in Each Diagnostic Category by Gender and Job Category*

\begin{tabular}{|c|c|c|c|c|c|c|}
\hline & & \multicolumn{5}{|c|}{ Men } \\
\hline & & \multicolumn{4}{|c|}{ Job Category } & \multirow[b]{2}{*}{ TOTAL } \\
\hline & & Professional & Technical Support & Service & Crafts & \\
\hline Diagnostic Category & ICD-9-CM Code & & & & & \\
\hline $\begin{array}{l}\text { NERVOUS SYSTEM } \\
\text { (NS) \& SENSE ORGANS }\end{array}$ & $320-389$ & 0 & 9 & 0 & 2 & 11 \\
\hline $\begin{array}{l}\text {-Disorders of Peripheral } \\
\text { NS }\end{array}$ & $350-359$ & 0 & 1 & 0 & 0 & 1 \\
\hline $\begin{array}{l}\text {-Diseases of } \\
\text { Ear \& Mastoid }\end{array}$ & $380-389$ & 0 & 8 & 0 & 2 & 10 \\
\hline $\begin{array}{l}\text { SKIN AND } \\
\text { SUBCUTANEOUS } \\
\text { TISSUE }\end{array}$ & 680-709 & 0 & 3 & 0 & 0 & 3 \\
\hline $\begin{array}{l}\text {-Other Inflammatory } \\
\text { Conditions }\end{array}$ & 690-698 & 0 & 3 & 0 & 0 & 3 \\
\hline $\begin{array}{l}\text { MUSCULOSKELETAL } \\
\text { \& CONNECTIVE } \\
\text { TISSUE }\end{array}$ & $710-739$ & 19 & 5 & 1 & 2 & 27 \\
\hline -Arthropathies & $710-719$ & 12 & 4 & 0 & 1 & 17 \\
\hline -Dorsopathies & $720-724$ & 4 & 1 & 1 & 0 & 6 \\
\hline $\begin{array}{l}\text {-Rheumatism, Excluding } \\
\text { Back }\end{array}$ & $725-729$ & 3 & 0 & 0 & 1 & 4 \\
\hline $\begin{array}{l}\text { SYMPTOMS, } \\
\text { SIGNS, \& ILL-DEFINED } \\
\text { CONDITIONS }\end{array}$ & $780-799$ & 1 & 1 & 0 & 0 & 2 \\
\hline -Symptoms & 780-789 & 1 & 1 & 0 & 0 & 2 \\
\hline INJURY \& POISONING & $800-999$ & 16 & 11 & 1 & 2 & 30 \\
\hline -Fracture - Lower Limb & $820-829$ & 1 & 0 & 0 & 0 & 1 \\
\hline -Sprains \& Strains - Back & 846-847 & 3 & 1 & 1 & 0 & 5 \\
\hline $\begin{array}{l}\text {-Sprains \& Strains - } \\
\text { Other }\end{array}$ & $840-845,848$ & 2 & 4 & 0 & 1 & 7 \\
\hline $\begin{array}{l}\text {-Open Wound - Upper } \\
\text { Limb }\end{array}$ & $880-887$ & 0 & 4 & 0 & 0 & 4 \\
\hline -Contusion & $920-924$ & 1 & 0 & 0 & 0 & 1 \\
\hline $\begin{array}{l}\text {-Complications \& } \\
\text { Unspecified Injuries }\end{array}$ & 958-959 & 7 & 2 & 0 & 1 & 10 \\
\hline $\begin{array}{l}\text {-Poisoning - Medicinal/ } \\
\text { Biological }\end{array}$ & $960-979$ & 1 & 0 & 0 & 0 & 1 \\
\hline $\begin{array}{l}\text {-Toxic } \\
\text { Effects - Non-medicinal }\end{array}$ & 980-989 & 1 & 0 & 0 & 0 & 1 \\
\hline
\end{tabular}

*Only those diagnostic categories and gender/job category combinations with at least one occurrence appear in this table. 
Lawrence Livermore National Laboratory 2010

OSHA Data

Appendix S. Number of Diagnoses in Each Diagnostic Category by Gender and Job Category*

\begin{tabular}{|c|c|c|c|c|c|}
\hline & \multicolumn{5}{|c|}{ Men } \\
\hline & \multicolumn{4}{|c|}{ Job Category } & \multirow[b]{2}{*}{ TOTAL } \\
\hline & Professional & Technical Support & Service & Crafts & \\
\hline Diagnostic Category & \multirow[b]{2}{*}{36} & \multirow[b]{2}{*}{29} & \multirow[b]{2}{*}{2} & \multirow[b]{2}{*}{6} & \multirow[b]{2}{*}{73} \\
\hline Total & & & & & \\
\hline
\end{tabular}

*Only those diagnostic categories and gender/job category combinations with at least one occurrence appear in this table. 


\section{Lawrence Livermore National Laboratory 2010}

OSHA Data

Appendix T. Number of Workdays Lost or with Restricted Activity in Each Diagnostic Category by Gender and Job Category*

\begin{tabular}{|c|c|c|c|c|c|c|c|}
\hline & & \multicolumn{6}{|c|}{ Women } \\
\hline & & \multicolumn{6}{|c|}{ Job Category } \\
\hline & & \multicolumn{2}{|c|}{ Professional } & \multicolumn{2}{|c|}{ Technical Support } & \multicolumn{2}{|c|}{ Service } \\
\hline & & $\begin{array}{c}\text { Days } \\
\text { Restricted }\end{array}$ & Days Lost & $\begin{array}{c}\text { Days } \\
\text { Restricted }\end{array}$ & Days Lost & $\begin{array}{c}\text { Days } \\
\text { Restricted }\end{array}$ & Days Lost \\
\hline Diagnostic Category & ICD-9-CM Codes & \multirow[b]{2}{*}{39} & \multirow[b]{2}{*}{94} & \multirow[b]{2}{*}{0} & \multirow[b]{2}{*}{0} & \multirow[b]{2}{*}{0} & \multirow[b]{2}{*}{0} \\
\hline -Disorders of Peripheral NS & $350-359$ & & & & & & \\
\hline -Arthropathies & $710-719$ & 195 & 115 & 0 & 0 & 23 & 0 \\
\hline -Dorsopathies & $720-724$ & 0 & 0 & 0 & 0 & 0 & 0 \\
\hline -Rheumatism, Excluding Back & $725-729$ & 0 & 105 & 0 & 0 & 0 & 0 \\
\hline -Symptoms & 780-789 & 0 & 11 & 0 & 0 & 0 & 0 \\
\hline -Fracture - Lower Limb & $820-829$ & 0 & 1 & 0 & 0 & 0 & 0 \\
\hline -Sprains \& Strains - Back & 846-847 & 0 & 0 & 0 & 0 & 0 & 0 \\
\hline -Sprains \& Strains - Other & $840-845,848$ & 170 & 13 & 0 & 0 & 0 & 0 \\
\hline -Open Wound - Lower Limb & 890-897 & 0 & 0 & 0 & 0 & 0 & 0 \\
\hline -Superficial Injury & $910-919$ & 0 & 0 & 0 & 0 & 0 & 0 \\
\hline -Contusion & $920-924$ & 0 & 0 & 0 & 0 & 0 & 0 \\
\hline -Burns & $940-949$ & 0 & 0 & 0 & 0 & 0 & 0 \\
\hline $\begin{array}{l}\text {-Complications \& Unspecified } \\
\text { Injuries }\end{array}$ & 958-959 & 32 & 50 & 0 & 0 & 23 & 0 \\
\hline -Toxic Effects - Non-medicinal & 980-989 & 0 & 11 & 0 & 0 & 0 & 0 \\
\hline
\end{tabular}

\begin{tabular}{|c|c|c|c|c|c|c|c|c|c|}
\hline & & \multicolumn{8}{|c|}{ Men } \\
\hline & & \multicolumn{8}{|c|}{ Job Category } \\
\hline & & \multicolumn{2}{|c|}{ Professional } & \multicolumn{2}{|c|}{ Technical Support } & \multicolumn{2}{|c|}{ Service } & \multicolumn{2}{|c|}{ Crafts } \\
\hline & & $\begin{array}{c}\text { Days } \\
\text { Restricted }\end{array}$ & $\begin{array}{l}\text { Days } \\
\text { Lost }\end{array}$ & $\begin{array}{c}\text { Days } \\
\text { Restricted }\end{array}$ & $\begin{array}{l}\text { Days } \\
\text { Lost }\end{array}$ & $\begin{array}{c}\text { Days } \\
\text { Restricted }\end{array}$ & $\begin{array}{l}\text { Days } \\
\text { Lost }\end{array}$ & $\begin{array}{c}\text { Days } \\
\text { Restricted }\end{array}$ & $\begin{array}{l}\text { Days } \\
\text { Lost }\end{array}$ \\
\hline Diagnostic Category & ICD-9-CM Codes & & & & & & & & \\
\hline -Disorders of Peripheral NS & $350-359$ & 0 & 0 & 0 & 0 & 0 & 0 & 0 & 0 \\
\hline -Diseases of Ear \& Mastoid & 380-389 & 0 & 0 & 0 & 0 & 0 & 0 & 0 & 0 \\
\hline -Other Inflammatory Conditions & $690-698$ & 0 & 0 & 0 & 0 & 0 & 0 & 0 & 0 \\
\hline -Arthropathies & $710-719$ & 0 & 7 & 158 & 58 & 0 & 0 & 0 & 0 \\
\hline -Dorsopathies & $720-724$ & 79 & 0 & 41 & 0 & 0 & 31 & 0 & 0 \\
\hline -Rheumatism, Excluding Back & 725-729 & 34 & 0 & 0 & 0 & 0 & 0 & 144 & 0 \\
\hline -Symptoms & 780-789 & 0 & 0 & 0 & 0 & 0 & 0 & 0 & 0 \\
\hline -Fracture - Lower Limb & 820-829 & 0 & 46 & 0 & 0 & 0 & 0 & 0 & 0 \\
\hline -Sprains \& Strains - Back & 846-847 & 79 & 0 & 41 & 0 & 0 & 31 & 0 & 0 \\
\hline -Sprains \& Strains - Other & $840-845,848$ & 0 & 0 & 285 & 71 & 0 & 0 & 0 & 0 \\
\hline -Open Wound - Upper Limb & $880-887$ & 0 & 0 & 3 & 10 & 0 & 0 & 0 & 0 \\
\hline -Contusion & $920-924$ & 0 & 1 & 0 & 0 & 0 & 0 & 0 & 0 \\
\hline $\begin{array}{l}\text {-Complications \& Unspecified } \\
\text { Injuries }\end{array}$ & 958-959 & 0 & 7 & 14 & 12 & 0 & 0 & 144 & 0 \\
\hline
\end{tabular}

(Continued)

*OSHA events with >1 ICD-9-CM code in the same diagnostic category were counted only once. Only those diagnostic categories and gender/job category combinations with at least one occurrence appear in this table. 
Lawrence Livermore National Laboratory 2010

OSHA Data

Appendix T. Number of Workdays Lost or with Restricted Activity in Each Diagnostic Category by Gender and Job Category*

\begin{tabular}{|c|c|c|c|c|c|c|c|c|c|}
\hline & & \multicolumn{8}{|c|}{ Men } \\
\hline & & \multicolumn{8}{|c|}{ Job Category } \\
\hline & & \multicolumn{2}{|c|}{ Professional } & \multicolumn{2}{|c|}{ Technical Support } & \multicolumn{2}{|c|}{ Service } & \multicolumn{2}{|c|}{ Crafts } \\
\hline & & $\begin{array}{c}\text { Days } \\
\text { Restricted }\end{array}$ & $\begin{array}{l}\text { Days } \\
\text { Lost }\end{array}$ & $\begin{array}{c}\text { Days } \\
\text { Restricted }\end{array}$ & $\begin{array}{l}\text { Days } \\
\text { Lost }\end{array}$ & $\begin{array}{c}\text { Days } \\
\text { Restricted }\end{array}$ & $\begin{array}{l}\text { Days } \\
\text { Lost }\end{array}$ & $\begin{array}{c}\text { Days } \\
\text { Restricted }\end{array}$ & $\begin{array}{l}\text { Days } \\
\text { Lost }\end{array}$ \\
\hline Diagnostic Category & ICD-9-CM Codes & \multirow[b]{2}{*}{0} & \multirow[b]{2}{*}{0} & \multirow[b]{2}{*}{0} & \multirow[b]{2}{*}{0} & \multirow[b]{2}{*}{0} & \multirow[b]{2}{*}{0} & \multirow[b]{2}{*}{0} & \multirow[b]{2}{*}{0} \\
\hline -Poisoning - Medicinal/Biological & $960-979$ & & & & & & & & \\
\hline -Toxic Effects - Non-medicinal & $980-989$ & 0 & 0 & 0 & 0 & 0 & 0 & 0 & 0 \\
\hline
\end{tabular}

*OSHA events with >1 ICD-9-CM code in the same diagnostic category were counted only once. Only those diagnostic categories and gender/job category combinations with at least one occurrence appear in this table. 


\section{Lawrence Livermore National Laboratory 2010}

OSHA Data

Appendix U. Number of Occurrences in Each Accident Category by Gender and Job Category*

\begin{tabular}{|c|c|c|c|c|c|}
\hline & & \multicolumn{4}{|c|}{ Women } \\
\hline & & \multicolumn{3}{|c|}{ Job Category } & \multirow[b]{2}{*}{ TOTAL } \\
\hline & & Professional & \begin{tabular}{|c|} 
Technical \\
Support
\end{tabular} & Service & \\
\hline Type of Accident & E CODES & \multirow[b]{2}{*}{1} & \multirow[b]{2}{*}{0} & \multirow[b]{2}{*}{0} & \multirow[b]{2}{*}{1} \\
\hline Other Road Vehicle & E826-E829 & & & & \\
\hline Accidental Poisoning - Non-medicinal & E860-E869 & 1 & 0 & 0 & 1 \\
\hline Falls & E880-E888 & 6 & 0 & 0 & 6 \\
\hline Other Accidents & E916-E928 & 19 & 3 & 1 & 23 \\
\hline
\end{tabular}

\begin{tabular}{|c|c|c|c|c|c|c|}
\hline & & \multicolumn{5}{|c|}{ Men } \\
\hline & & \multicolumn{4}{|c|}{ Job Category } & \multirow[b]{2}{*}{ TOTAL } \\
\hline & & Professional & $\begin{array}{l}\text { Technical } \\
\text { Support }\end{array}$ & Service & Crafts & \\
\hline Type of Accident & E CODES & \multirow[b]{2}{*}{1} & \multirow[b]{2}{*}{0} & \multirow[b]{2}{*}{0} & \multirow[b]{2}{*}{0} & \multirow[b]{2}{*}{1} \\
\hline Other Road Vehicle & E826-E829 & & & & & \\
\hline $\begin{array}{l}\text { Accidental } \\
\text { Poisoning - Medicinal/Biological }\end{array}$ & E850-E858 & 1 & 0 & 0 & 0 & 1 \\
\hline Falls & E880-E888 & 2 & 1 & 0 & 0 & 3 \\
\hline Natural/Environmental Factors & E900-E909 & 1 & 0 & 0 & 0 & 1 \\
\hline Other Accidents & E916-E928 & 12 & 18 & 1 & 4 & 35 \\
\hline
\end{tabular}

*Only those accident types and gender/job category combinations with at least one occurrence appear in this table. 
Lawrence Livermore National Laboratory 2010

OSHA Data

Appendix V. Number of Workdays Lost or with Restricted Activity in Each Accident Category by Gender and Job Category*

\begin{tabular}{|c|c|c|c|c|c|c|c|}
\hline & & \multicolumn{6}{|c|}{ Women } \\
\hline & & \multicolumn{6}{|c|}{ Job Category } \\
\hline & & \multicolumn{2}{|c|}{ Professional } & \multicolumn{2}{|c|}{ Technical Support } & \multicolumn{2}{|c|}{ Service } \\
\hline & & $\begin{array}{c}\text { Days } \\
\text { Restricted }\end{array}$ & Days Lost & $\begin{array}{c}\text { Days } \\
\text { Restricted }\end{array}$ & Days Lost & $\begin{array}{c}\text { Days } \\
\text { Restricted }\end{array}$ & Days Lost \\
\hline Type of Accident & E Codes & \multirow[b]{2}{*}{0} & \multirow[b]{2}{*}{0} & \multirow[b]{2}{*}{0} & \multirow[b]{2}{*}{0} & \multirow[b]{2}{*}{0} & \multirow[b]{2}{*}{0} \\
\hline Other Road Vehicle & E826-E829 & & & & & & \\
\hline Accidental Poisoning - Non-medicinal & E860-E869 & 0 & 11 & 0 & 0 & 0 & 0 \\
\hline Falls & E880-E888 & 0 & 3 & 0 & 0 & 0 & 0 \\
\hline Other Accidents & E916-E928 & 241 & 260 & 0 & 0 & 23 & 0 \\
\hline
\end{tabular}

\begin{tabular}{|c|c|c|c|c|c|c|c|c|c|}
\hline & & \multicolumn{8}{|c|}{ Men } \\
\hline & & \multicolumn{8}{|c|}{ Job Category } \\
\hline & & \multicolumn{2}{|c|}{ Professional } & \multicolumn{2}{|c|}{ Technical Support } & \multicolumn{2}{|c|}{ Service } & \multicolumn{2}{|c|}{ Crafts } \\
\hline & & $\begin{array}{c}\text { Days } \\
\text { Restricted }\end{array}$ & $\begin{array}{l}\text { Days } \\
\text { Lost }\end{array}$ & $\begin{array}{c}\text { Days } \\
\text { Restricted }\end{array}$ & $\begin{array}{l}\text { Days } \\
\text { Lost }\end{array}$ & $\begin{array}{c}\text { Days } \\
\text { Restricted }\end{array}$ & $\begin{array}{l}\text { Days } \\
\text { Lost }\end{array}$ & $\begin{array}{c}\text { Days } \\
\text { Restricted }\end{array}$ & $\begin{array}{l}\text { Days } \\
\text { Lost }\end{array}$ \\
\hline Type of Accident & E Codes & \multirow[b]{2}{*}{0} & \multirow[b]{2}{*}{46} & \multirow[b]{2}{*}{0} & \multirow[b]{2}{*}{0} & \multirow[b]{2}{*}{0} & \multirow[b]{2}{*}{0} & \multirow[b]{2}{*}{0} & \multirow[b]{2}{*}{0} \\
\hline Other Road Vehicle & E826-E829 & & & & & & & & \\
\hline $\begin{array}{l}\text { Accidental } \\
\text { Poisoning - Medicinal/Biological }\end{array}$ & E850-E858 & 0 & 0 & 0 & 0 & 0 & 0 & 0 & 0 \\
\hline Falls & E880-E888 & 0 & 1 & 141 & 35 & 0 & 0 & 0 & 0 \\
\hline Natural/Environmental Factors & E900-E909 & 0 & 0 & 0 & 0 & 0 & 0 & 0 & 0 \\
\hline Other Accidents & E916-E928 & 113 & 7 & 202 & 58 & 0 & 31 & 144 & 0 \\
\hline
\end{tabular}

*OSHA events with >1 E code in the same accident type were counted only once. Only those accident types and gender/job category combinations with at least one occurrence appear in this table. 


\section{Lawrence Livermore National Laboratory 2010}

OSHA Data

Appendix W. Age-Adjusted OSHA Illness and Injury Rates by Diagnostic Category*

Part 1. Men

\begin{tabular}{|c|c|c|c|c|c|}
\hline & & $\begin{array}{l}\text { Number of } \\
\text { Diagnoses }\end{array}$ & $\begin{array}{l}\text { Age-Adjusted } \\
\text { Rate per } 1,000 * *\end{array}$ & $\begin{array}{c}\text { Lower } 95 \% \\
\text { Confidence } \\
\text { Limit per 1,000 }\end{array}$ & $\begin{array}{c}\text { Upper } 95 \% \\
\text { Confidence } \\
\text { Limit per 1,000 }\end{array}$ \\
\hline Diagnostic Category & ICD-9-CM Code & \multirow[b]{2}{*}{11} & \multirow[b]{2}{*}{1.3} & \multirow[b]{2}{*}{0.7} & \multirow[b]{2}{*}{2.3} \\
\hline NERVOUS SYSTEM (NS) \& SENSE ORGANS & 320-389 & & & & \\
\hline -Disorders of Peripheral NS & 350-359 & 1 & 0.1 & 0.0 & 0.7 \\
\hline -Diseases of Ear \& Mastoid & 380-389 & 10 & 1.2 & 0.6 & 2.2 \\
\hline SKIN AND SUBCUTANEOUS TISSUE & $680-709$ & 3 & 0.4 & 0.1 & 1.3 \\
\hline -Other Inflammatory Conditions & 690-698 & 3 & 0.4 & 0.1 & 1.3 \\
\hline $\begin{array}{l}\text { MUSCULOSKELETAL \& CONNECTIVE } \\
\text { TISSUE }\end{array}$ & 710-739 & 27 & 3.9 & 2.6 & 5.9 \\
\hline -Arthropathies & $710-719$ & 17 & 2.2 & 1.3 & 3.6 \\
\hline -Dorsopathies & 720-724 & 6 & 0.9 & 0.4 & 2.4 \\
\hline -Rheumatism, Excluding Back & $725-729$ & 4 & 0.8 & 0.3 & 2.0 \\
\hline $\begin{array}{l}\text { SYMPTOMS, SIGNS, \& ILL-DEFINED } \\
\text { CONDITIONS }\end{array}$ & 780-799 & 2 & 0.2 & 0.0 & 0.8 \\
\hline -Symptoms & $780-789$ & 2 & 0.2 & 0.0 & 0.8 \\
\hline INJURY \& POISONING & $800-999$ & 30 & 4.0 & 2.7 & 5.9 \\
\hline -Fracture - Lower Limb & 820-829 & 1 & 0.1 & 0.0 & 0.7 \\
\hline -Sprains \& Strains - Back & 846-847 & 5 & 0.8 & 0.3 & 2.4 \\
\hline -Sprains \& Strains - Other & $840-845,848$ & 7 & 0.9 & 0.4 & 1.9 \\
\hline -Open Wound - Upper Limb & 880-887 & 4 & 0.5 & 0.2 & 1.4 \\
\hline -Contusion & $920-924$ & 1 & 0.1 & 0.0 & 0.7 \\
\hline -Toxic Effects - Non-medicinal & $980-989$ & 1 & 0.2 & 0.0 & 1.4 \\
\hline -Complications of Surgical/Medical Care & 996-999 & 11 & 1.4 & 0.8 & 2.7 \\
\hline Total & & 73 & 9.7 & 7.5 & 12.5 \\
\hline
\end{tabular}

* Only those diagnostic categories with at least one occurrence appear in this table.

**Standardized to age distribution of 2000 U.S. population. 


\section{Lawrence Livermore National Laboratory 2010}

OSHA Data

Appendix W. Age-Adjusted OSHA IIlness and Injury Rates by Diagnostic Category*

Part 2. Women

\begin{tabular}{|c|c|c|c|c|c|}
\hline & & $\begin{array}{l}\text { Number of } \\
\text { Diagnoses }\end{array}$ & $\begin{array}{c}\text { Age-Adjusted } \\
\text { Rate per 1,000** }\end{array}$ & $\begin{array}{c}\text { Lower } 95 \% \\
\text { Confidence } \\
\text { Limit per 1,000 }\end{array}$ & $\begin{array}{c}\text { Upper } 95 \% \\
\text { Confidence } \\
\text { Limit per 1,000 }\end{array}$ \\
\hline Diagnostic Category & ICD-9-CM Code & \multirow[b]{2}{*}{3} & \multirow[b]{2}{*}{0.8} & \multirow[b]{2}{*}{0.3} & \multirow[b]{2}{*}{2.7} \\
\hline NERVOUS SYSTEM (NS) \& SENSE ORGANS & 320-389 & & & & \\
\hline -Disorders of Peripheral NS & $350-359$ & 3 & 0.8 & 0.3 & 2.7 \\
\hline $\begin{array}{l}\text { MUSCULOSKELETAL \& CONNECTIVE } \\
\text { TISSUE }\end{array}$ & 710-739 & 25 & 8.4 & 5.4 & 12.9 \\
\hline -Arthropathies & 710-719 & 14 & 5.0 & 2.8 & 8.8 \\
\hline -Dorsopathies & $720-724$ & 2 & 0.5 & 0.1 & 1.8 \\
\hline -Rheumatism, Excluding Back & 725-729 & 9 & 2.9 & 1.4 & 6.1 \\
\hline $\begin{array}{l}\text { SYMPTOMS, SIGNS, \& ILL-DEFINED } \\
\text { CONDITIONS }\end{array}$ & 780-799 & 6 & 2.1 & 0.8 & 5.3 \\
\hline -Symptoms & $780-789$ & 6 & 2.1 & 0.8 & 5.3 \\
\hline INJURY \& POISONING & $800-999$ & 31 & 19.6 & 10.2 & 37.6 \\
\hline -Fracture - Lower Limb & 820-829 & 2 & 0.5 & 0.1 & 1.8 \\
\hline -Sprains \& Strains - Back & 846-847 & 1 & 0.4 & 0.1 & 2.7 \\
\hline -Sprains \& Strains - Other & $840-845,848$ & 4 & 1.2 & 0.4 & 3.4 \\
\hline -Open Wound - Lower Limb & 890-897 & 1 & 0.8 & 0.1 & $\overline{5.6}$ \\
\hline -Superficial Injury & $910-919$ & 1 & 0.8 & 0.1 & 5.6 \\
\hline -Contusion & $920-924$ & 3 & 2.3 & 0.7 & 7.1 \\
\hline -Burns & $940-949$ & 1 & 5.9 & 0.8 & 41.7 \\
\hline -Toxic Effects - Non-medicinal & $980-989$ & 1 & 0.2 & 0.0 & 1.6 \\
\hline -Complications of Surgical/Medical Care & 996-999 & 17 & 7.6 & 4.5 & 13.0 \\
\hline Total & & 65 & 30.9 & 20.0 & 47.7 \\
\hline
\end{tabular}

* Only those diagnostic categories with at least one occurrence appear in this table.

**Standardized to age distribution of 2000 U.S. population. 


\section{Lawrence Livermore National Laboratory 2010}

OSHA Data

Appendix W. Age-Adjusted OSHA Illness and Injury Rates by Diagnostic Category*

Part 3. Men and Women

\begin{tabular}{|c|c|c|c|c|c|}
\hline & & $\begin{array}{c}\text { Number of } \\
\text { Diagnoses }\end{array}$ & $\begin{array}{c}\text { Age-Adjusted } \\
\text { Rate per } 1,000 * *\end{array}$ & $\begin{array}{c}\text { Lower } 95 \% \\
\text { Confidence } \\
\text { Limit per 1,000 }\end{array}$ & $\begin{array}{c}\text { Upper } 95 \% \\
\text { Confidence } \\
\text { Limit per 1,000 }\end{array}$ \\
\hline Diagnostic Category & ICD-9-CM Code & & & & \\
\hline NERVOUS SYSTEM (NS) \& SENSE ORGANS & 320-389 & 14 & 1.1 & 0.7 & 2.0 \\
\hline -Disorders of Peripheral NS & $350-359$ & 4 & 0.3 & 0.1 & 0.9 \\
\hline -Diseases of Ear \& Mastoid & 380-389 & 10 & 0.8 & 0.4 & 1.5 \\
\hline SKIN AND SUBCUTANEOUS TISSUE & $680-709$ & 3 & 0.3 & 0.1 & 0.9 \\
\hline -Other Inflammatory Conditions & $690-698$ & 3 & 0.3 & 0.1 & 0.9 \\
\hline $\begin{array}{l}\text { MUSCULOSKELETAL \& CONNECTIVE } \\
\text { TISSUE }\end{array}$ & 710-739 & 52 & 5.4 & 4.0 & 7.3 \\
\hline -Arthropathies & $710-719$ & 31 & 3.2 & 2.2 & 4.7 \\
\hline -Dorsopathies & $720-724$ & 8 & 0.8 & 0.4 & 1.8 \\
\hline -Rheumatism, Excluding Back & 725-729 & 13 & 1.4 & 0.8 & 2.6 \\
\hline $\begin{array}{l}\text { SYMPTOMS, SIGNS, \& ILL-DEFINED } \\
\text { CONDITIONS }\end{array}$ & 780-799 & 8 & 0.8 & 0.3 & 1.8 \\
\hline -Symptoms & 780-789 & 8 & 0.8 & 0.3 & 1.8 \\
\hline INJURY \& POISONING & $800-999$ & 61 & 8.4 & 5.7 & 12.5 \\
\hline -Fracture - Lower Limb & $820-829$ & 3 & 0.2 & 0.1 & 0.6 \\
\hline -Sprains \& Strains - Back & 846-847 & 6 & 0.7 & 0.3 & 1.8 \\
\hline -Sprains \& Strains - Other & $840-845,848$ & 11 & 1.0 & 0.5 & 1.9 \\
\hline -Open Wound - Upper Limb & 880-887 & 4 & 0.3 & 0.1 & 0.9 \\
\hline -Open Wound - Lower Limb & 890-897 & 1 & 0.3 & 0.0 & 1.8 \\
\hline -Superficial Injury & 910-919 & 1 & 0.3 & 0.0 & 1.8 \\
\hline -Contusion & $920-924$ & 4 & 0.7 & 0.2 & 2.1 \\
\hline -Burns & $940-949$ & 1 & 1.3 & 0.2 & 9.2 \\
\hline -Toxic Effects - Non-medicinal & 980-989 & 2 & 0.2 & 0.0 & 0.8 \\
\hline -Complications of Surgical/Medical Care & 996-999 & 28 & 3.4 & 2.3 & 5.3 \\
\hline Total & & 138 & 16.1 & 12.7 & 20.3 \\
\hline
\end{tabular}

* Only those diagnostic categories with at least one occurrence appear in this table.

**Standardized to age distribution of 2000 U.S. population. 